\title{
PROCESS PHYSICS
}

\author{
Reginald T. Cahill
}

\section{School of Chemistry, Physics and Earth Sciences}

Flinders University

GPO Box 2100, Adelaide 5001, Australia

\author{
Reg.Cahill@flinders.edu.au \\ Process Physics URLs: \\ http://www.mountainman.com.au/process_physics/ \\ http://www.scieng.flinders.edu.au/cpes/people/cahill_r/processphysics.html
}

Process Studies Supplement URL:

http://www.ctr4process.org/publications/PSS/index.htm 
Process Studies Supplement $2003 \quad$ Issue $5 \quad$ R.T. Cahill.......Process Physics 2

\begin{abstract}
This is a review of the new information-theoretic Process Physics. This new modelling of reality brings physics very much into accord with the general concepts of Process Philosophy. The fundamental assumption is that reality is to be modelled as self-organising semantic or relational information using a self-referentially limited neural network model, where the information-theoretic limitations are implemented via self-referential noise. This modelling was motivated by the discovery that such stochastic neural networks are foundational to known quantum field theories. In Process Physics time is a distinct nongeometric process while space and quantum physics are emergent and unified. Quantum phenomena are caused by fractal topological defects embedded in and forming a growing three-dimensional fractal process-space, which is essentially a quantum foam. Other features are the emergence of quantum field theory with flavour and confined colour, limited causality and the Born quantum measurement metarule, inertia, time-dilation effects, gravity and the equivalence principle, a growing universe with a cosmological constant, black holes and event horizons, and the emergence of classicality. The unification of the quantum foam structure of space with the quantum nature of matter amounts to the discovery of quantum gravity. Gravity is essentially an in-flow effect associated with the loss of information. A new theory of gravity for the classical limit is proposed, and shown to pass the key tests. A detailed analysis of various experiments demonstrates that absolute motion with respect to this space of quantum foam has been observed in the interferometer experiments of Michelson and Morley, Miller, Illingworth, Joos, Jaseja et $a l$ and in the coaxial cable experiments by Torr and Kolen, and by DeWitte. The Miller data also reveal the in-flow of space into matter which manifests as gravity. The in-flow also manifests turbulence and the experimental data of Miller, DeWitte, and Torr and Kolen confirms this, which amounts to the observation of a gravitational wave phenomena. Contrary to the usual assumptions absolute motion is consistent with relativistic effects, which are caused by actual dynamical effects of absolute motion through the quantum foam, so that it is Lorentzian relativity that is seen to be essentially correct. Special relativity effects are the key to understanding and analysing the interferometer fringe shift data, together with the refractive index effect. The success of this new physics has profound implications for our comprehension of reality, and supersedes the current non-Process Physics modelling of reality.
\end{abstract}




\section{Contents}

1 Introduction $\quad 6$

2 Non-Process and Process Physics 9

2.1 Non-Process Physics . . . . . . . . . . . . . . . . . . . 9

2.2 Process Physics . . . . . . . . . . . . . . . . . . . . 11

$3 \quad$ Syntactical and Semantic Information Systems 13

3.1 Self-Referential Systems and Gödel's Theorem . . . . . . . . . . . . 13

3.2 Semantic Information System . . . . . . . . . . . . . . . . . . 15

3.3 Process Philosophy . . . . . . . . . . . . . 16

4 Self-Referentially Limited Neural Networks $\quad 18$

4.1 Bootstrapping a Semantic Information System . . . . . . . . . . . . . 18

4.2 Stochastic Neural Networks from Quantum Field Theory . . . . . . . . . 20

4.3 Neural Networks . . . . . . . . . . . . . . . . . . . . . . . 22

4.4 Emergent Geometry in Stochastic Neural Network: Gebits . . . . . . . . 24

$5 \quad$ Gebits and their Topological Defects $\quad 27$

5.1 Gebits as Skyrmions . . . . . . . . . . . . . . . . 27

5.2 Absence of a Cosmic Code . . . . . . . . . . . . . . . . . 28

5.3 Entrapped Topological Defects . . . . . . . . . . . . . 28

6 Quantum Homotopic Field Theory 29

6.1 Functional Schrödinger Equation . . . . . . . . . . . . . . . . 29

6.2 Homotopy Hamiltonian . . . . . . . . . . . . . . . . . . . . . . . . 31

6.3 Quantum State Diffusion . . . . . . . . . . . . . . . . . 32

6.4 Emergent Classicality . . . . . . . . . . . . . . . . . . 32

7 Emergent Quantum Field Theory of Matter $\quad 34$

7.1 Quantum Field Theory . . . . . . . . . . . . . . . 34

7.2 Emergent Flavour and Hidden Colour . . . . . . . . . . . . . . . 34

8 Emergent Universe 35

8.1 Multi-World Universe and the 'Dark Energy' Effect . . . . . . . . . . . . 35

8.2 Gravity and the Equivalence Principle . . . . . . . . . . . . . 36

8.3 Inertia . . . . . . . . . . . . . . . 37 
9 Quantum Foam Flow and a New Theory of Gravity 38

9.1 Classical Effects of Quantum Foam . . . . . . . . . . . . . . . 39

9.2 The Einstein Measurement Protocol . . . . . . . . . . . . . . . . . . . . . . . . 43

9.3 The Origins of General Relativity . . . . . . . . . . . . . . 46

9.4 Deconstruction of General Relativity . . . . . . . . . . . . . . . . 48

9.5 Gravity as Inhomogeneous Quantum-Foam Flow . . . . . . . . . . . 50

9.6 The 'Dark Matter' Effect . . . . . . . . . . . . . . . . . . 51

9.7 In-Flow Superposition Approximation . . . . . . . . . . . . . . 52

9.8 Gravitational In-Flow and the GPS . . . . . . . . . . . . . . 53

9.9 Measurements of $G \ldots \ldots \ldots \ldots \ldots$

10 Detection of Absolute Motion $\quad 57$

10.1 Space and Absolute Motion . . . . . . . . . . . . . . 57

10.2 Theory of the Michelson Interferometer . . . . . . . . . . . . . . 62

10.3 The Michelson-Morley Experiment: $1887 \ldots \ldots$. . . . . . . . . . 66

10.4 The Miller Interferometer Experiment: 1925-1926 . . . . . . . . . . . 71

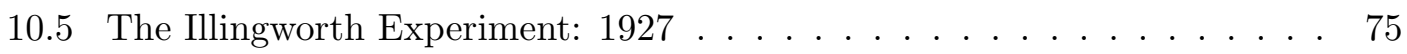

10.6 The Joos Experiment: $1930 \ldots \ldots \ldots \ldots$. . . . . . . . . 76

10.7 The New Bedford Experiment: $1963 \ldots \ldots \ldots$. . . . . . . 77

10.8 The DeWitte Experiment: $1991 \ldots \ldots$. . . . . . . . . . . 81

10.9 The Torr-Kolen Experiment: $1981 \ldots \ldots$. . . . . . . . . 87

11 Gravitational In-Flow and Gravitational Waves $\quad 89$

11.1 Gravitational In-flow from the Miller Data . . . . . . . . . . . . . . . . 89

11.2 Galactic In-flow and the CMB Frame . . . . . . . . . . . . . . . . . . . 91

11.3 In-Flow Turbulence and Gravitational Waves . . . . . . . . . . . . . . . . . . . 93

11.4 Gravitational Anomalies . . . . . . . . . . . . . . . . . . . . . . . . . . . . . . . . . 95

11.5 The Borehole g Anomaly . . . . . . . . . . . . . . . . . . . . . . . . . . . . . 96

11.6 Absolute Motion and Quantum Gravity . . . . . . . . . . . . . . . . . 98

12 Modern Interferometers 100

12.1 Vacuum Michelson Interferometers . . . . . . . . . . . . . . . 100

12.2 Solid-State Michelson Interferometers . . . . . . . . . . . . . . . . . 101

12.3 New Absolute Motion Detectors . . . . . . . . . . . . . . . . 102

13 Looking Back 105

13.1 The Failure of the Einstein Postulates . . . . . . . . . . . . . . . 105

13.2 The Shankland Paper: 1955 . . . . . . . . . . . . . . . 109

14 Conclusions $\quad 111$ 
Process Studies Supplement 2003 Issue $5 \quad$ R.T. Cahill......Process Physics 5

15 Acknowledgments

16 Appendices $\quad 115$

16.1 Gebit Connectivity . . . . . . . . . . . . . . . . . 115

16.2 Fresnel Drag . . . . . . . . . . . . . . . . . . . . 118

16.3 Michelson Interferometer Analysis - no Fresnel Drag . . . . . . . . . . . 119

16.4 Michelson Interferometer Analysis - with Fresnel Drag . . . . . . . . 120

16.5 Michelson Interferometer and the Einstein Postulates . . . . . . . . . . . 122

17 References 


\section{Introduction}

Process Physics is an information-theoretic modelling of reality. It is a new physics. This paper presents a collection of significant new developments as well as a review of previously reported developments, and so it amounts to a consolidation of the new emerging paradigm. Most importantly the new developments are supported by extensive analysis of existing data from numerous experiments going back to the enormously influential Michelson-Morley experiment of 1887. Process Physics is radically different from the current Non-Process Physics modelling of reality, and the experimental data analysed herein clearly refutes much of the foundations of Non-Process Physics. Non-Process Physics is essentially the reality paradigm associated with the names of Galileo, Newton, Einstein and many others. Its most distinctive feature is that it models time by geometry, and although superficially it has been very successful it is demonstrated here that much of the so-called 'experimental confirmation' of its foundational assumptions has in fact been nothing more than a consequence of major misunderstandings in experimental and theoretical physics. Process Physics on the other hand is distinguished by modelling time as process. It arose from various considerations including:

(i) the belief that the geometrical modelling of time was deficient, that it simply missed most aspects of the phenomena of time, see Griffin [1] for an overview,

(ii) the discovery that 'beneath' the quantum theory of matter known as quantum field theory there were the elements of a stochastic neural-network, that is, a mathematical system that has some resemblance to the mathematical modelling of the neural networks of biological systems. This suggested that reality was probably generating and processing information as patterns, and not as symbols in some axiomatic formal system, and that this was 'semantic information', that is having 'meaning' within the system, and

(iii) the Gödel limitations of formal information systems in which the information is syntactical and so has no 'meaning' within the system. Non-Process Physics is such a syntactical system.

Over a number of recent years these ideas were moulded into a new paradigm for reality in which much of the success of the Non-Process Physics paradigm was recovered, but most significantly it became apparent for the first time why reality is the way it is, rather than as in the previous paradigm, a mere mimicry of reality encoded in formalisms and 'laws'. It turns out that by taking account of the Gödel limitations we realise the end of an old paradigm and the emergence of a much richer paradigm.

The Process Physics paradigm has in fact a long and illustrious history beginning most notably with the ideas of Heraclitus of Ephesus who should perhaps be regarded as the first process physicist. This paradigm has had many supporters and collectively 
it became known as Process Philosophy, as distinct from its non-process competitor Analytic Philosophy. Within Process Philosophy the most distinguished contributors were Peirce, James, Bergson and Whitehead to name a few. A collection of their writings is available in [2]. Recently Eastman and Keeton [3] have produced a resource guide to work within the general area of process physics and with an emphasis on the work of Whitehead, see also [4].

The successes of Process Physics are already impacting on the foundations of physics with much support evident in existing experimental data. The new physics is also already motivating new experiments as would be expected of any new paradigm. It is becoming clear now how to design experiments that can and will distinguish the older Non-Process Physics from the new Process Physics. As well this new physics will have a major impact outside of the traditional boundaries of science, where it offers particular insights into our attempts to comprehend the phenomena of consciousness, and more broadly the nature of living systems. If these insights are also supported by new scientific endeavours then we can expect significant changes in our appreciation of our own human nature and its many manifestations in social and religious areas. Indeed Process Physics may unsnarl what Schopenhauer called the world-knot [5]. These potential developments will not be canvassed here as this work is focused exclusively on bringing the new paradigm to investigation and acceptance by the traditional-science community. For that reason this work is both theoretically and experimentally detailed for without a penetrating analysis the claims for the new physics could not be sustained. Nevertheless process philosophers will find that the emerging insights will strongly resonate with the many more general notions that are current within process philosophy.

The collapse of the older non-process paradigm is also exposing dramatic human aspects of the historical development of that paradigm, for the failure of that paradigm is actually traceable to numerous bungles and flawed human behaviour. Indeed some of that history is shockingly scandalous, and in particular the ongoing behaviour of the physics profession is very disturbing - basically the non-process paradigm has acquired the status of a belief system, as distinct from a science, and as such is defended with ruthless and unscrupulous behaviour. For many the non-process paradigm is now beyond challenge, and both old and new experiments that contradicted that paradigm are simply to be ignored and even suppressed. Indeed the many true successes of the old paradigm have ultimately led to nothing more than stagnation and decay, and much current work in theoretical physics has degenerated to spurious mathematical generalisations of a failed paradigm.

The consolidation so far of the new process physics is evident in the table of contents. In general the work moves from the logic of the limitations of logic to the bootstrapping of a semantic information system. This bootstrapping system possesses none of the 
known phenomena of current physics, but in later sections we see the emergence of space and quantum matter in a unified manner. The characteristics of the emergent space are different from that of the modelling of space within the geometrical paradigm and in particular as well as absolute motion, that is motion with respect to space itself. A new and detailed theory of gravity arises. Essentially ever since the Einsteins ${ }^{1}$ proposed that absolute motion was without meaning and so unobservable, following Poincaré, the physicists have essentially banned the concept, and it remains as such today. This state of affairs can be traced to a key misunderstanding that follows from the Einstein postulates; namely that absolute motion is incompatible with relativistic effects. However there is extensive experimental evidence, which is assembled and analysed here for the first time, that absolute motion has been observed again and again, and was clearly evident even in the seminal Michelson-Morley experiment. Over the years the only experiments that were permitted to be published in the physics literature were those that actually failed to observe absolute motion. Not because they were poorly executed but simply because in principle they were actually unable to detect the phenomena of absolute motion. The experiments that detected and studied absolute motion, and amongst these the most significant was the impressive series by Miller, now reveal evidence in support of the new theory of gravity. Gravity is neither a force nor a curvature of spacetime, but rather inhomogeneities in the effective in-flow of the quantum foam that is space into matter. This in-flow and even its associated turbulence are manifest in the experimental data from various absolute motion experiments. As a consequence we clearly see that the Einstein curved spacetime construct is without experimental support, that it actually never was confirmed by the key and celebrated experiments, for they were actually confirming another explanation. In the end we see that the Einstein spacetime formalism is demonstrably wrong, and that the evidence against it was available even before the Einsteins put forward their fundamental assumptions that later became the foundations of twentieth century physics. Essentially the whole Einstein formalism has all the hallmarks of another system of 'epicycles' - when finally we understand what is going on the whole construct evaporates, just as Ptolemy's epicycles did when it was realised that they were entirely a consequence of not separating a measurement protocol from the phenomena it was meant to measure. In the case of Ptolemy it was finally realised that the earth was itself undergoing motion. In the case of the Einstein formalism we finally understand that the rods and clocks used to define and implement measurements of motion are actually affected by motion through the quantum foam that is space, a view that predated the Einsteins and is now seen to be correct.

All of these developments and the clearing away of epicycle descriptions lead us back to very challenging notions about the nature of time and the deep connectivity

\footnotetext{
${ }^{1}$ Referring to Einstein and his then wife Mileva Maric-Einstein.
} 
and processing that is reality, a connectivity that was evident in some aspects of the quantum theory, but which was essentially outside of the non-process paradigm. This new physics is seen to be panexperientialist in character in which a primitive self-awareness or 'consciousness' is foundational to reality in the manner argued by Griffin and others [5], a consciousness that appears to be intrinsic to the semantic nature of the information system that is process physics. Such notions it seems may well be moving into the realm of experimental science and will result in a unification of human knowledge and experience that is beyond our prevailing comprehensions.

\section{Non-Process and Process Physics}

\subsection{Non-Process Physics}

Non-Process Physics had its genesis with the introduction by Galileo of a geometrical modelling of the phenomena of time. At first this seems quite a strange model as geometry has little in common with time. Time has three aspects, and briefly they are (i) that there is a present moment that (ii) separates the past from the future, and (iii) the past is fixed and at best recorded, while the future is undecided and certainly not recorded. The only aspect of time that the geometrical model captures is that of order: the sequence of points along a one-dimensional geometrical line have the property that if $A<B$ and $B<C$, then $A<C$, where $A, B, C, \ldots$ are points on the line, and the relation $A<B$ means "A is to the left of B", as in Fig.1. To model time this geometrical order property of points is used to represent the time order of events: $A, B, C \ldots$ now represent events, and $A<B$ means that event $A$ is or was "before" event $B$. However the model has no means of modelling the present moment effect - there is no moving point on the line that we could identify as Now.

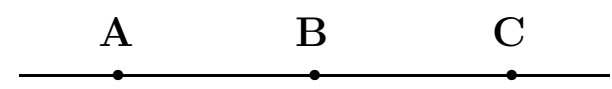

Figure 1: Modelling the order aspect of the phenomena of time by the ordering of points along a one-dimensional geometrical line.

As a consequence of that the model is unable to capture the distinction between the past and the future, and even worse the identification of $<$ with time ordering is not unique, as we could also have chosen to use $A<B$ to mean " $A$ was after $B$ ". Despite this mismatching this modelling of time proved to be exceptionally useful, particularly after 
the metric property of the line was used to quantify time intervals: the distance $d(A, B)$ between $A$ and $B$ along the line is used to represent the time interval $t(A, B)$ between events $A$ and $B$. The efficacy of this followed when it was demonstrated by Galileo that the quantification of time intervals actually was useful in kinematical observations of penudula and other experimental phenomena, and that regular oscillations of for example a pendulum could be used to measure $t(A, B)$. Newton developed this model much further by showing that it could be used to model the dynamics of motion as well, as for example in the famous equation

$$
m \frac{d^{2} x(t)}{d x^{2}}=F(t)
$$

which not only uses the geometrical model of time, as in the symbol $t$, but also uses the older modelling of space by the Euclidean geometrical model, as in the symbol $x$ (here we show only one of the three dimensions of space). Eqn.(1) also illustrates the use of the calculus that was independently developed by Leibniz and Newton. As well the symbols $m$ and $F$ refer to the mass of the object undergoing motion and the force acting on that mass, respectively. We offer later an explanation for inertia, as measured by this $m$.

The overwhelming success of the paradigm of reality encapsulated in (1) eventually led most physicists to actually believe that the phenomena of time was really nothing more than geometry, just as they had believed that the phenomena of space was really geometry. The way physicists got around the obvious lack of the phenomena of the Now, the present moment effect, was to imagine that the present moment was thought of as a point moving along the line. This is an example of a metarule, here the geometrical time metarule: it plays no part in the analysis of (1) but seems to make the model better match up with reality.

One would have expected that the manifest deficiencies of the geometrical nonprocessing model of time would eventually have resulted in extensive research by physicists to develop a better model, and perhaps even one that could mimic all the aspects of the phenomena of time. But this simply did not happen. In the twentieth century we know that this did not happen because of again the influence of the Einsteins. The ill-conceived attempt to formulate a model of reality in which absolute motion was without meaning resulted in the introduction of the spacetime construct. This is a fourdimensional geometrical construct in which the one-dimensional model of time is fused with the three-dimensional model of space, but fused in a special way in that observers in relative motion would identify different foliations of the construct as their geometrical time lines, so that their modellings of time no longer coincided, and as a consequence they could no longer necessarily agree on the time-ordering of events. Rather than being seen as an indicator of something wrong in the model this aspect of the spacetime model became a celebrated feature, and the whole notion of change, of the evolution of reality 
from a past state to a future state disappeared, and reality it was claimed was simply a frozen unchanging four-dimensional block of geometry: when the universe was formed the whole of the future of that universe also popped into existence. So what about our experiences of the present moment and the distinction between past and future? Well that was dismissed as being some trick of our minds, and a trick that psychologists should investigate, but certainly not physicists. As will become clear later the Einstein postulates that led to this bizarre spacetime construct of reality are simply proven wrong by experiment: absolute motion is an observable feature of reality and experiments that show this have been carried out many times. As a consequence time is an aspect of reality that is distinct from the phenomena of space, and neither are at a deep level geometrical systems.

\subsection{Process Physics}

Process Physics $[6,7,8,9,10,11,12,13,14,15,16,17,18,19]$ is a radical informationtheoretic modelling of reality which arose from analysis of various extant limitations; from the limitations of formal information systems discovered by Gödel, Turing and Chaitin, from the limitations of the geometric modelling of time in the models constructed by Galileo, Newton and Einstein, and by the limitations of the quantum theory and its failure to account for the measurement process. As is usual within physics these limitations were obscured by various metarules and metaphysical constructs. This informationtheoretic modelling was also motivated by the discovery that stochastic neural network models are foundational to known quantum field theories $[6,7]$.

In process physics the fundamental assumption is that reality is to be modelled as self-organising semantic information, that is, information that is 'internally' meaningful, using a self-referentially limited neural network model. Such a system has no a priori objects or laws, and is evolved using a bootstrap system, so that it is the system itself that 'internally' creates patterns of relationships and their dominant modes of behaviour, and all (sub)systems are fractal in character, that is, relationships within relationships, and so on ad infinitum. In this way all emergent phenomena are unified, and it is this key feature that has resulted in an understanding and linking, for the first time, of various phenomena. A key feature of this process-physics is that this fracticality is associated with self-organising criticality.

Previously it was shown that space and quantum physics are emergent and unified, with time a distinct non-geometric process, that quantum phenomena are caused by fractal topological defects embedded in and forming a growing three-dimensional fractal process-space, which is essentially a quantum foam. Other features of the emergent physics were quantum field theory with emergent flavour and confined colour, limited 
causality and the Born quantum measurement metarule, inertia, time-dilation effects, gravity and the equivalence principle, a growing universe with a cosmological constant, black holes and event horizons, and the emergence of classicality.

The emergence of a quantum-foam explanation for space is accompanied by the effect in which quantum 'matter' effectively acts as a sink for the quantum foam. This provides an explanation for the logical necessity of the phenomenon of gravity. Essentially gravity is the loss of relational information during the in-flow to matter. The presently accepted theory of gravity is General Relativity (GR) with its key concept of curved spacetime. Here it is shown that GR is a confused amalgam of the Einstein measurement protocol with nothing more than Newtonian Gravity (NG). The quantum foam in-flow effect that is gravity is seen not to be identical to GR for as shown here GR actually is identical to Newtonian gravity in all the cases where it has been tested experimentally. Significantly we reveal strong experimental and theoretical considerations which indicate that the fundamental foundations of GR appear to be erroneous. The Einstein assumptions on which the Special and General Theory of Relativity are based are shown to be in disagreement with numerous experiments. They are demonstrably wrong ${ }^{2}$. Even more surprisingly is that Newtonian Gravity (NG) appears to be valid only in special circumstances, and that the phenomena of gravity is in fact very different from the expectations of NG and GR. It is argued here that the anomalous rotational speed effect in spiral galaxies is a manifestation of the failure of both Newtonian and the Hilbert-Einstein gravity. A new theory of gravity is presented in provisional form. This theory passes all the tests that GR had apparently passed, but predicts other phenomena that are not predicted by GR or even NG. This spacetime construct is shown here to have no ontological significance though it does have technical uses.

The ongoing failure of physics to fully match all the aspects of the phenomena of time, apart from that of order, arises because physics has always used non-process models, as is the nature of formal or syntactical systems. Such systems do not require any notion of process - they are entirely structural and static. The new process physics overcomes these deficiencies by using a non-geometric process model for time, but process physics also argues for the importance of relational or semantic information in modelling reality. Semantic information refers to the notion that reality is a purely informational system where the information is internally meaningful. Hence the information is 'content addressable', rather than is the case in the usual syntactical information modelling

\footnotetext{
${ }^{2}$ There is considerable debate, discussed later, about who developed the foundational ideas that resulted in the formulation of SR and GR. The key new insight offered here is that aside from this historical analysis the resulting GR is actually wrong, while the interpretation of SR is inappropriate, and amounted to an obscurification rather than a contribution to the development and understanding of relativistic effects.
} 
where the information is represented by symbols. This symbolic or syntactical mode is only applicable to higher level phenomenological descriptions, and for that reason was discovered first.

A key long-standing goal of physics has been the unification of gravity and the quantum theory. This unification could not be achieved within the old non-process physics, but has now been achieved with the new process physics. The quantum theory arises from the non-geometric and non-quantum self-referentially-limited neural network model which implements the semantic information approach to comprehending reality. What has been achieved in process physics is the explanation of why reality must be so, and why the modes of behaviour are encodeable in the syntax of the non-process physics. This older mode of dealing with reality will continue to be used because for many problems it is eminently practical. It will require the continued use of various metarules to overcome its limitations, but we now have an explanation for them as well.

Process Physics shows that there is a Quantum Gravity, but it is unrelated to General Relativity and General Covariance, and essentially describes the emergent quantum phenomena of the process-space or quantum foam, and its response to quantum 'matter', and all this within an information-theoretic framework. This Quantum Gravity is manifested in the emergent Quantum Homotopic Field Theory for the process-space or quantum foam. Clearly it would be absurd to attempt the 'quantisation' of GR, not only because 'quantisation' is a dubious idea at best, but because GR is a fundamentally flawed theory of gravity.

A pure semantic information system must be formed by a subtle bootstrap process. The mathematical model for this has the form of a stochastic neural network (SNN) for the simple reason that neural networks are well known for their pattern or nonsymbolic information processing abilities [20,21]. The stochastic behaviour is related to the limitations of syntactical systems discovered by Gödel [22] and more recently extended by Chaitin $[23,24,25]$, but also results in the neural network being innovative in that it creates its own patterns. The neural network is self-referential, and the stochastic

input, known as self-referential noise, acts both to limit the depth of the self-referencing and also to generate potential order.

\section{Syntactical and Semantic Information Systems}

\subsection{Self-Referential Systems and Gödel's Theorem}

In modelling reality with formal or syntactical information systems physicists assume that a full account of reality can be compressed into axioms and rules for the manipulation of symbols. However Gödel discovered that self-referential syntactical systems (and these 
include basic mathematics) have fundamental limitations which amount to the realisation that not all truths can be compressed into an axiomatic structure, that formal systems are much weaker than previously supposed. In physics such systems have always been used in conjunction with metarules and metaphysical assertions, all being 'outside' the formal system and designed to overcome the limitations of the syntax. Fig.2 depicts the current understanding of self-referential syntactical systems. Here the key feature is the Gödel boundary demarcating the provable from the unprovable truths of some system. Chaitin, using Algorithmic Information Theory, has demonstrated that in mathematics the unprovable truths are essentially random in character. This, however, is a structural randomness in the sense that the individual truths do not have any structure to them which could be exploited to condense them down to or be encoded in axioms. This is unlike random physical events which occur in time. Of course syntactical systems are based on the syntax of symbols and this is essentially non-process or non-timelike.

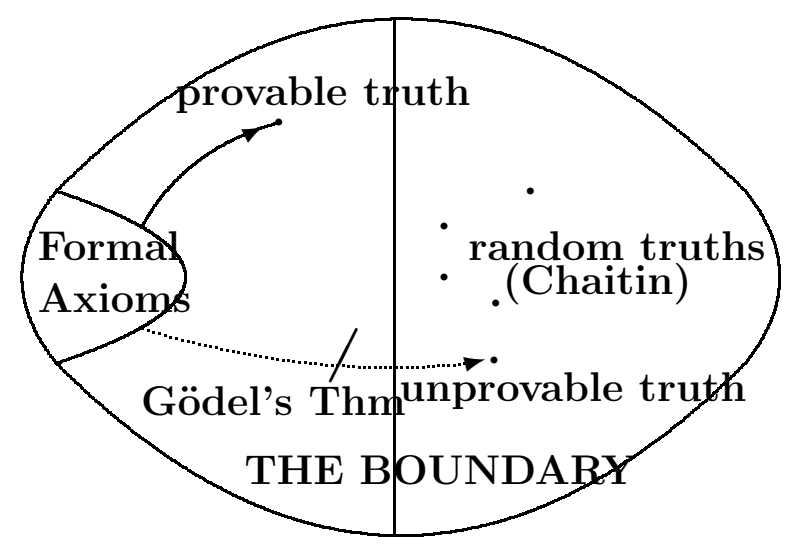

Figure 2: Graphical depiction of the 'logic space' of a self-referential syntactical information system, showing the formal system consisting of symbols and rules, and an example of one theorem (a provable truth). Also shown are unprovable truths which in general are random (or unstructured) in character, following the work of Chaitin. The Gödelian boundary is the demarcation between provable and unprovable truths.

There is an analogy between the structure of self-referential syntactical information systems and the present structure of quantum theory, as depicted in Fig.3. There the formal and hence non-process mathematical structure is capable of producing many provable truths, such as the energy levels of the hydrogen atom, and these are also true in the sense that they agree with reality. But from the beginning of quantum theory the Born measurement metarule was introduced to relate this non-process modelling 


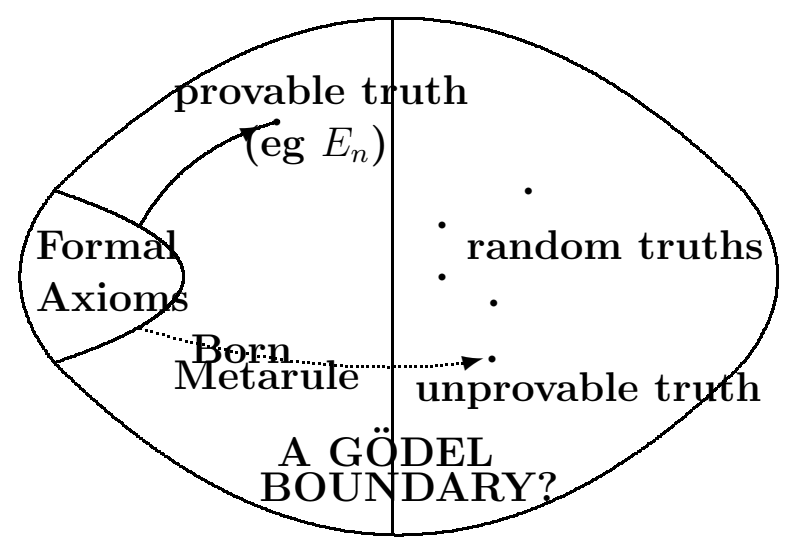

Figure 3: Graphical depiction of the syntactical form of conventional quantum theory. The Born measurement metarule appears to bridge a Gödel-like boundary.

to the actual randomness of quantum measurement events. The individuality of such random events is not a part of the formal structure of quantum theory. Of course it is well known that the non-process or structural aspects of the probability metarule are consistent with the mathematical formalism, in the form of the usual 'conservation of probability' equation and the like. Further, the quantum theory has always been subject to various metaphysical interpretations, although these have never played a key role for practitioners of the theory. This all suggests that perhaps the Born metarule is bridging a Gödel-type boundary, that there is a bigger system required to fully model quantum aspects of reality, and that the boundary is evidence of self-referencing in that system.

\subsection{Semantic Information System}

Together the successes and failures of physics suggest that a generalisation beyond the traditional use of syntactical information theory is required to model reality. This has now been identified as a semantic information system which is modelled as a stochastic neural network.

Fig.4 shows a graphical depiction of the bootstrapping of a pure semantic information system, showing the stochastic neural network-like process system from which the semantic system is seeded or bootstrapped. Via a Self-Organised Criticality Filter (SOCF) this seeding system is removed or hidden. From the process system, driven by Self-Referential Noise (SRN), there are emergent truths, some of which are generically true (ensemble truths) while others are purely contingent. The ensemble truths are also reachable from the Induced Formal System as theorems, but from which, because of the non-process 


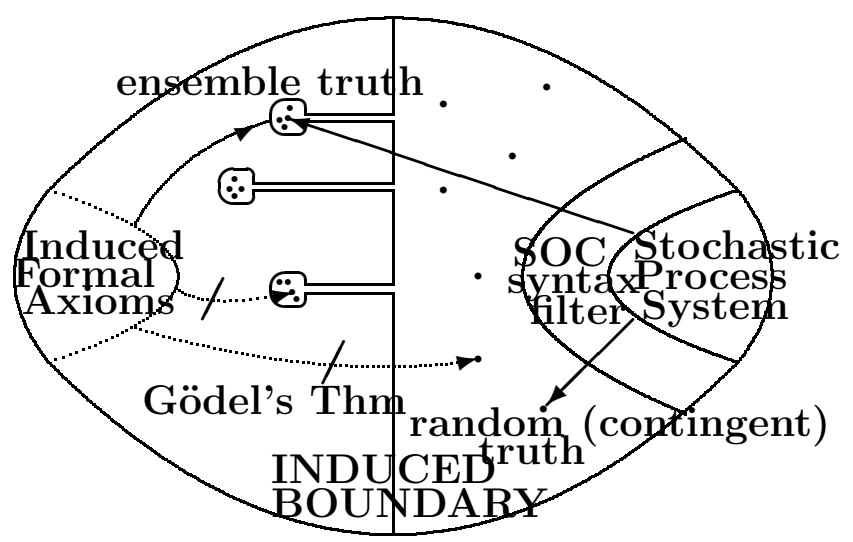

Figure 4: Graphical depiction of the bootstrapping of and the emergent structure of a self-organising pure semantic information system. As a high level effect we see the emergence of an induced formal system, corresponding to the current standard syntactical modelling of reality. There is an emergent Gödel-type boundary which represents the inaccessibility of the random or contingent truths from the induced formal or syntactical system. A process of self-organised criticality (SOC) filters out the seeding or bootstrap syntax.

nature of the Induced Formal System, the contingent truths cannot be reached. In this manner there arises a Gödel-type boundary. The existence of the latter leads to induced metarules that enhance the Induced Formal System, if that is to be used solely in higher order phenomenology. A detailed account of a Sematic Information System is given in Sect.4.

\subsection{Process Philosophy}

Western science and philosophy have always been dominated by non-process thought. This 'historical record' or being model of reality has been with us since Parmenides, and his student Zeno of Elea, and is known as the Eleatic model (c500 BCE). Zeno gave us the first insights into the inherent problems of comprehending motion, a problem long forgotten by conventional non-process physics, but finally explained by process physics. The becoming or processing model of reality dates back to Heraclitus of Ephesus (540-480 BCE) who argued that common sense is mistaken in thinking that the world consists of stable things; rather the world is in a state of flux. The appearances of 'things' depend upon this flux for their continuity and identity. What needs to be explained, Heraclitus argued, is not change, but the appearance of stability. With process physics western science and philosophy is now able to move beyond the moribund non-process mindset. But it was the work of Gödel that demonstrated beyond any doubt that the non-process 
system of thought had fundamental limitations; implicit in his work is the idea that the whole reductionist mindset that goes back to Thales of Miletus could not offer, in the end, an effective account of reality. However the notion that there were limits to syntactical or symbolic encoding is actually very old. Priest [26] has given an account of that history. However in the East the Buddhists in particular were amazingly advanced in their analysis and comprehension of reality. Stcherbatsky [27], writing about the extraordinary achievements of Buddhist logic in the $\mathrm{C} 6$ and $\mathrm{C} 7^{\text {th }} \mathrm{CE}$, noted that

Reality according to Buddhists is kinetic, not static, but logic, on the other hand, imagines a reality stabilized in concepts and names. The ultimate aim of Buddhist logic is to explain the relation between a moving reality and the static constructions of logic.

In the West the process system approach to reality was developed, much later, by such process philosophers as Peirce, James, Bergson and Whitehead to name a few, although their achievements were very limited and substantially flawed, limited as they were by the physical phenomena known to them. A collection of their writings is available in [2]. Perhaps a quote from Charles Peirce [2], writing in 1891, gives the sense of their thinking;

The one intelligible theory of the universe is that of objective idealism, that matter is effete mind, inveterate habits becoming physical laws. But before this can be accepted it must show itself capable of explaining the tridimensionalty of space, the laws of motion, and the general characteristics of the universe, with mathematical clearness and precision; for no less should be demanded of every philosophy.

With process physics we have almost achieved this end, and Wheeler has already expressed this notion of inveterate habits as "law without law" [28]. As the reader will note the self-referentially limited neural network model, that underpins process physics, is remarkably akin to Peirce's effete mind. It is the limitations of syntax, and the need for intrinsic or semantic information 'within' reality and at all levels, that reality is not imposed, that drives us to this approach. Einstein, the modern day eleatic thinker, realised all too well the limitations of non-process thinking but was unable to move out of the non-process realm that the West had created for itself, for according to Carnap $[29]$;

Once Einstein said that the problem of the Now worried him seriously. He explained that the experience of the Now means something special for man, something essentially different from the past and the future, but that this 
important difference does not and cannot occur within physics. That this experience cannot be grasped by science seems to him a matter of painful but inevitable resignation. I remarked that all that occurs objectively can be described in science: on the one hand the temporal sequence of events is described in physics; and, on the other hand, the peculiarities of man's experiences with respect to time, including his different attitude toward past, present and future, can be described and (in principle) explained in psychology. But Einstein thought that scientific descriptions cannot possibly satisfy our human needs; that there is something essential about the Now which is just outside of the realm of science.

It was the Einstein's error in rejecting absolute motion that trapped twentieth century physics in the non-process or no now mindset. As is shown here experiments that could detect absolute motion did so, and those that could not not do so in principle of course did not detect absolute motion. Nevertheless all of these latter experiments were claimed to have confirmed the SR and GR formalism which is fundamentally based an the absence of absolute motion as an aspect of reality.

\section{Self-Referentially Limited Neural Networks}

\subsection{Bootstrapping a Semantic Information System}

Here we describe a model for a self-referentially limited neural network and in the following sections we see how such a network results in emergent geometry and quantum behaviour, and which, increasingly, appears to be a unification of space and quantum phenomena. Process physics is a semantic information system and is devoid of a priori objects and their laws and so it requires a subtle bootstrap mechanism to set it up. We use a stochastic neural network, Fig.5a, having the structure of real-number valued connections or relational information strengths $B_{i j}$ (considered as forming a square matrix) between pairs of nodes or pseudo-objects $i$ and $j$. In standard neural networks $[20,21]$ the network information resides in both link and node variables, with the semantic information residing in attractors of the iterative network. Such systems are also not pure in that there is an assumed underlying and manifest a priori structure.

The nodes and their link variables will be revealed to be themselves sub-networks of informational relations. To avoid explicit self-connections $B_{i i} \neq 0$ which are a part of the sub-network content of $i$, we use antisymmetry $B_{i j}=-B_{j i}$ to conveniently ensure that $B_{i i}=0$, see Fig.5b.

At this stage we are using a syntactical system with symbols $B_{i j}$ and, later, rules for the changes in the values of these variables. This system is the syntactical seed for the 
pure semantic system. Then to ensure that the nodes and links are not remnant a priori objects the system must generate strongly linked nodes (in the sense that the $B_{i j}$ for these nodes are much larger than the $B_{i j}$ values for non- or weakly-linked nodes) forming a fractal network; then self-consistently the start-up nodes and links may themselves be considered as mere names for sub-networks of relations. For a successful suppression the scheme must display self-organised criticality (SOC) [32] which acts as a filter for the start-up syntax. The designation 'pure' refers to the notion that all seeding syntax has been removed. SOC is the process where the emergent behaviour displays universal criticality in that the behaviour is independent of the particular start-up syntax; such a start-up syntax then has no ontological significance.

To generate a fractal structure we must use a non-linear iterative system for the $B_{i j}$ values. These iterations amount to the necessity to introduce a time-like process. Any system possessing a priori 'objects' can never be fundamental as the explanation of such objects must be outside the system. Hence in process physics the absence of intrinsic undefined objects is linked with the phenomena of time, involving as it does an ordering of 'states', the present moment effect, and the distinction between past and present. Conversely in non-process physics the necessity for a priori objects is related to the use of the non-process geometrical model of time, with this modelling and its geometricaltime metarule being an approximate emergent description from process-time. In this way process physics arrives at a new modelling of time, process time, which is much more complex than that introduced by Galileo, developed by Newton, and reaching its so called high point but deeply flawed Einstein spacetime geometrical model. Unlike these geometrical models process-time does model the Now effect. Process physics also shows that time cannot be modelled by any other structure, other than a time-like process, here an iterative scheme. There is nothing like time available for its modelling. The near obsession of theoretical physicists with the geometrical modelling of time, and its accompanying notion of analytical determinism, has done much to retard the development of physics. The success of process physics implies that time along with self-referencing is in some sense prior to the other phenomena, and certainly prior to space, as will be seen in Sect.(8) within the discussion of a multi-component universe.

The stochastic neural network so far has been realised with one particular scheme involving a stochastic non-linear matrix iteration, see (2). The matrix inversion $B^{-1}$ then models self-referencing in that it requires all elements of $B$ to compute any one element of $B^{-1}$. As well there is the additive Self-Referential Noise (SRN) $w_{i j}$ which limits the self-referential relational information but, significantly, also acts in such a way that the network is innovative in the sense of generating semantic information, that is relational information which is internally meaningful. The emergent behaviour is believed to be completely generic in that it is not suggested that reality is a computation, rather it 


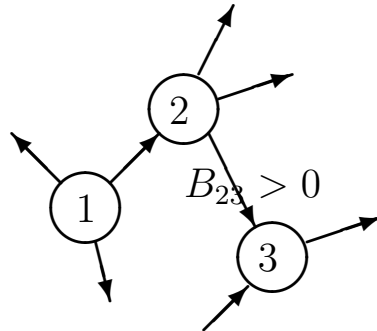

(a)

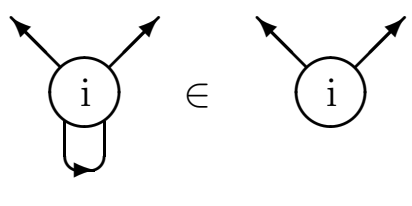

(b)

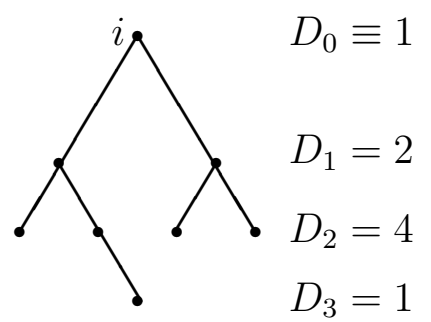

(c)

Figure 5: (a) Graphical depiction of the neural network with links $B_{i j} \in \mathcal{R}$ between nodes or pseudo-objects. Arrows indicate sign of $B_{i j}$. (b) Self-links are internal to a node, so $B_{i i}=0$. (c) An $N=8$ spanning tree for a random graph (not shown) with $L=3$. The distance distribution $D_{k}$ is indicated for node $i$.

appears that reality has the form of a self-referential order-disorder information system. It is important to note that process physics is a non-reductionist modelling of reality; the basic iterator (2) is premised on the general assumption that reality is sufficiently complex that self-referencing occurs, and that this has limitations. Eqn.(2) is then a minimal bootstrapping implementation of these notions. At higher emergent levels this self-referencing manifests itself as interactions between emergent patterns, but other novel effects may also arise.

To be a successful contender for the Theory of Everything (TOE) process physics must ultimately prove the uniqueness conjecture: that the characteristics (but not the contingent details) of the pure semantic information system are unique. This would involve demonstrating both the effectiveness of the SOC filter and the robustness of the emergent phenomenology, and the complete agreement of the latter with observation.

The stochastic neural network is modelled by the iterative process

$$
B_{i j} \rightarrow B_{i j}-\alpha\left(B+B^{-1}\right)_{i j}+w_{i j}, \quad i, j=1,2, \ldots, 2 N ; N \rightarrow \infty,
$$

where $w_{i j}=-w_{j i}$ are independent random variables for each $i j$ pair and for each iteration and chosen from some probability distribution. Here $\alpha$ is a parameter the precise value of which should not be critical but which influences the self-organisational process.

\subsection{Stochastic Neural Networks from Quantum Field The- ory}

It may be helpful to outline the thoughts that led to (2), arising as it did from the quantum field theory frontier of quark physics. A highly effective approximation to Quantum Chromodynamics (QCD) was developed that made extensive use of bilocal fields and 
the functional integral calculus (FIC), see [30] for reviews of this Global Colour Model (GCM). In the GCM the bilocal-field correlators (giving meson and baryon correlators) are given by the generating functional

$$
Z[J]=\int \mathcal{D} B^{\theta} \exp \left(-S[B]+\int d x^{4} d^{4} y B^{\theta}(x, y) J^{\theta}(x, y)\right) .
$$

Here $x, y \in E^{4}$, namely a Euclidean-metric space-time, as the hadronic correlators are required for vacuum-to-vacuum transitions, and as is well known the use of the Euclidean metric picks out the vacuum state of the quantum field theory. The physical Minkowskimetric correlators are then obtained by analytic continuation $x_{4} \rightarrow i x_{0}$. Eqn.(3) follows from (approximately) integrating out the gluon variables, and then changing variables from the quark Grassmannian functional integrations to bilocal-field functional integrations. Here the $\theta$ index labels generators of flavour, colour and spin. This form is well suited to extracting hadronic phenomena as the vacuum state of QCD corresponds to a BCS-type superconducting state, with the $q \bar{q}$ Cooper pairs described by those non-zero mean-field $\bar{B}^{\theta}(x, y)$ determined by the Euler-Lagrange equations of the action,

$$
\left.\frac{\delta S[B]}{\delta B^{\theta}(x, y)}\right|_{\bar{B}^{\theta}(x, y)}=0 .
$$

That (4) has non-zero solutions is the constituent-quark/BCS-state effect. This is nonlinear equation for those non-zero bilocal fields about which the induced effective action for hadronic fields is to be expanded.

Rather than approximately evaluating (3) as a functional integral, as done in [30], we may use the Parisi-Wu [31] stochastic 'quantisation' procedure, which involves the Langevin iterative equation

$$
B^{\theta}(x, y) \rightarrow B^{\theta}(x, y)-\frac{\delta S[B]}{\delta B^{\theta}(x, y)}+w^{\theta}(x, y)
$$

where $w^{\theta}(x, y)$ are Gaussian random variables with zero means. After many iterations a statistical equilibrium is achieved, and the required hadronic correlators may be obtained by statistical averaging: $\left\langle B^{\theta}(x, y) B^{\phi}(u, v) \ldots>\right.$, but with again analytic continuation back to Minkowski metric required. In particular, writing $B^{\theta}(x, y)=\phi\left(\frac{x+y}{2}\right) \Gamma(x-$ $\left.y, \frac{x+y}{2}\right)$, then $\phi(x)$ is a meson field, while $\Gamma(x, X)$ is the meson form factor.

That (5) leads to quantum behaviour is a remarkable result. The presence of the noise means that the full structure of $S[B]$ is explored during the iterations, whereas in (3) this is achieved by integration over all values of the $B^{\theta}(x, y)$ variables. The correlators $<B^{\theta}(x, y) B^{\phi}(u, v) \ldots>$ correspond to complex quantum phenomena involving bound states of constituent quarks embedded in a BCS superconducting state. However the Euclidean-metric $E^{4}$-spacetime plays a completely classical and passive background role. 
Now (5) has the form of a stochastic neural network (see later), with link variables $B^{\theta}(x, y)$, that is, with the nodes being continuously distributed in $E^{4}$. An interesting question arises: if we strip away the passive classical $E^{4}$ background and the superscript indices, so that $B^{\theta}(x, y) \rightarrow B_{i j}$ and we retain only a simple form for $S[B]$, then does this discretised Langevin equation, in (2), which now even more so resembles a stochastic neural network, continue to display quantum behaviour? In series of papers $[6,9,10,11]$ it has been found that indeed the SNN in (2) does exhibit quantum behaviour, by generating a quantum-foam dynamics for an emergent space, and with quantum -'matter' being topological-defects embedded in that quantum-foam in a unification of quantum space and matter. Indeed the remarkable discovery is that (2) generates a quantum gravity. Note, however, that now the iterations in (2) correspond to physical time, and we do not wait for equilibrium behaviour. Indeed the non-equilibrium behaviour manifests as a growing universe. The iterations correspond to a non-geometric modelling of time with an intrinsic arrow of time, as the iterations in (2) cannot be reversed. Hence the description of this new physics as Process Physics.

If (2) does in fact lead to a unification of gravity and quantum theory, then the deep question is how should we interpret (2)? The stochastic noise has in fact been interpreted as the new intrinsic Self-Referential Noise when the connection with the work of Gödel and Chaitin became apparent, and as discussed above. Hence beneath quantum field theory there is evidence of a self-referential stochastic neural network, and its interpretation as a semantic information system. Only by discarding the spacetime background of QFT do we discover the necessity for space and the quantum.

\subsection{Neural Networks}

We now briefly compare this iterator to an Attractor Neural Network (ANN) and illustrate its basic mode of operation (see [20,21] for details of neural networks). An ANN has link $J_{i j} \in \mathcal{R}$ and node $s_{i}= \pm 1$ variables $(i, j=1,2, \ldots N)$, with $J_{i j}=J_{j i}$ and $J_{i i}=0$. Here $s=+1$ denotes an active node, while $s=-1$ denotes an inactive node. The time evolution of the nodes is given by, for example,

$$
s_{i}(t)=\operatorname{sign}\left(\sum_{j} J_{i j} s_{j}(t-1)\right) .
$$

To imprint a pattern its $s_{i} \propto \xi_{i}$ values are imposed on the nodes and the Hebbian Rule is used to change the link strengths

$$
J_{i j}(t)=J_{i j}(t-1)+c s_{i}(t-1) s_{j}(t-1),
$$


and for $p$ successively stored patterns $\left(\xi^{1}, \xi^{2}, \ldots \xi^{p}\right)$ we end up with

$$
J_{i j}=\sum_{\mu=1}^{p} \xi_{i}^{\mu} \xi_{j}^{\mu}, \quad i \neq j .
$$

The imprinted patterns correspond to local minima of the energy function

$$
E[\{s\}]=-\frac{1}{2} \sum J_{i j} s_{i} s_{j}
$$

which has basins of attraction when the ANN is 'exposed' to an external input $s_{i}(0)$. As is well known over iterations of (6) the ANN node variables converges to one of the stored patterns most resembling $s_{i}(0)$. Hence the network categorises the external input.

The iterator (2), however, has no external inputs and its operation is determined by the detailed interplay between the order/disorder terms. As well it has no node variables: whether a node $i$ is active is determined implicitly by $\left|B_{i j}\right|>s$, for some $j$, where $s$ is some minimum value for the link variables. Because $B_{i j}$ is antisymmetric and real its eigenvalues occur in pairs $i b,-i b$ ( $b$ real), with a complete set of orthonormal eigenvectors $\xi^{\mu}, \mu= \pm 1, \pm 2, . ., \pm N,\left(\xi^{\mu *}=\xi^{-\mu}\right)$ so that

$$
B_{j k}=\sum_{\mu= \pm 1, \pm 2, . .} i b_{\mu} \xi_{j}^{\mu} \xi_{k}^{\mu *}, \quad b_{\mu}=-b_{-\mu} \in \mathcal{R}
$$

where the coefficients must occur in conjugate pairs for real $B_{i j}$. This corresponds to the form

$$
B=M D M^{-1}, \quad D=\left(\begin{array}{rrrrr}
0 & +b_{1} & 0 & 0 & \\
-b_{1} & 0 & 0 & 0 & \\
0 & 0 & 0 & +b_{2} & \\
0 & 0 & -b_{2} & 0 & \\
& & & & .
\end{array}\right)
$$

where $M$ is a real orthogonal matrix. Both the $b_{\mu}$ and $M$ change with each iteration.

Let us consider, in a very unrealistic situation, how patterns can be imprinted unchanged into the SNN. This will only occur if we drop the $B^{-1}$ term in (1). Suppose the SRN is frozen (artificially) at the same form on iteration after iteration. Then iterations of (2) converge to

$$
B=\frac{1}{\alpha} w=\sum_{\mu} i \alpha^{-1} w_{\mu} \eta_{j}^{\mu} \eta_{k}^{\mu *}
$$

where $w^{\mu}$ and $\eta^{\mu}$ are the eigensystem for $w$. This is analogous to the Hebbian rule (7), and demonstrates the imprinting of $w$, which is strong for small $\alpha$. If the noise is now 'turned-off' then this imprinted pattern will decay, but do so slowly if $\alpha$ is small. Hence to maintain an unchanging imprinted pattern it needs to be continually refreshed via a 
fixed $w$. However the iterator with the $B^{-1}$ term present has a significantly different and richer mode of behaviour as the system will now generate novel patterns, rather than simply imprinting whatever pattern is present in $w$. Indeed the system uses special patterns (the gebits) implicit in a random $w$ that are used as a resource with which much more complex patterns are formed.

The task is to determine the nature of the self-generated patterns, and to extract some effective descriptive syntax for that behaviour, remembering that the behaviour is expected to be quantum-like.

\subsection{Emergent Geometry in Stochastic Neural Network: Gebits}

We start the iterations of $(2)$ at $B \approx 0$, representing the absence of information, that is, of patterns. With the noise absent the iterator behaves in a deterministic and reversible manner giving a condensate-like system with a $B$ matrix of the form in (11), but with the matrix $M$ iteration independent amd determined uniquely by the start-up $B$, and each $b_{\mu}$ evolves according to the iterator $b_{\mu} \rightarrow b_{\mu}-\alpha\left(b_{\mu}-b_{\mu}^{-1}\right)$, which converges to $b_{\mu}= \pm 1$. The corresponding eigenvectors $\xi^{\mu}$ do not correspond to any meaningful patterns as they are determined entirely by the random values from the start-up $B \approx 0$. However in the presence of the noise the iterator process is non-reversible and non-deterministic and, most importantly, non-trivial in its pattern generation. The iterator is manifestly nongeometric and non-quantum in its structure, and so does not assume any of the standard features of syntax based non-process physics models. Nevertheless, as we shall see, it generates geometric and quantum behaviour. The dominant mode is the formation of an apparently randomised background (in $B$ ) but, however, it also manifests a self-organising process which results in non-trivial patterns which have the form of a growing threedimensional fractal process-space displaying quantum-foam behaviour. These patterns compete with this random background and represent the formation of a 'universe'.

The emergence of order in this system might appear to violate expectations regarding the 2nd Law of Thermodynamics; however because of the SRN the system behaves as an open system and the growth of order arises from the self-referencing term, $B^{-1}$ in (2), selecting certain implicit order in the SRN. Hence the SRN acts as a source of negentropy. The term negentropy was introduced by E. Schrödinger in 1945 [33], and since then there has been ongoing discussion of its meaning. In process physics it manifests as the SRN.

This growing three-dimensional fractal process-space is an example of a Prigogine far-from- equilibrium dissipative structure [34] driven by the SRN. From each iteration the noise term will additively introduce rare large value $w_{i j}$. These $w_{i j}$, which define sets of strongly linked nodes, will persist through more iterations than smaller valued $w_{i j}$ and, 
as well, they become further linked by the iterator to form a three-dimensional processspace with embedded topological defects. In this way the stochastic neural-network creates stable strange attractors and as well determines their interaction properties. This information is all internal to the system; it is the semantic information within the network.

To see the nature of this internally generated information consider a node $i$ involved in one such large $w_{i j}$; it will be connected via other large $w_{i k}$ to a number of other nodes and so on, and this whole set of connected nodes forms a connected random graph unit which we call a gebit as it acts as a small piece or bit of geometry formed from random information links and from which the process-space is self-assembled. The gebits compete for new links and undergo mutations. Indeed process physics is remarkably analogous in its operation to biological systems. The reason for this is becoming clear: both reality and subsystems of reality must use semantic information processing to maintain existence, and symbol manipulating systems are totally unsuited to this need, and in fact totally contrived.

To analyse the connectivity of such gebits assume for simplicity that the large $w_{i j}$ arise with fixed but very small probability $p$, then the emergent geometry of the gebits is revealed by studying the probability distribution for the structure of the random graph units or gebits minimal spanning trees with $D_{k}$ nodes at $k$ links from node $i\left(D_{0} \equiv 1\right)$, see Fig.5c, this is given by (see Sect.16.1 for derivation or [35]);

$$
\mathcal{P}\left[D, L, N_{g}\right] \propto \frac{p^{D_{1}}}{D_{1} ! D_{2} ! \ldots . . D_{L} !} \prod_{i=1}^{L-1}\left(q^{\sum_{j=0}^{i-1} D_{j}}\right)^{D_{i+1}}\left(1-q^{D_{i}}\right)^{D_{i+1}},
$$

where $q=1-p, N_{g}$ is the total number of nodes in the gebit and $L$ is the maximum depth from node $i$. To find the most likely connection pattern we numerically maximise $\mathcal{P}\left[D, L, N_{g}\right]$ for fixed $N_{g}$ with respect to $L$ and the $D_{k}$. The resulting $L$ and $\left\{D_{1}, D_{2}, \ldots, D_{L}\right\}$ fit very closely to the form $D_{k} \propto \sin ^{d-1}(\pi k / L)$; see Fig.6a for $N_{g}=5000$ and $\log _{10} p=-6$. The resultant $d$ values for a range of $\log _{10} p$ and with $N_{g}=5000$ are shown in Fig.6b.

This shows, for $p$ below a critical value, that $d=3$, indicating that the connected nodes have a natural embedding in a $3 \mathrm{D}$ hypersphere $S^{3}$; call this a base gebit. Above that value of $p$, the increasing value of $d$ indicates the presence of extra links that, while some conform with the embeddability, others are in the main defects with respect to the geometry of the $S^{3}$. These extra links act as topological defects. By themselves these extra links will have the connectivity and embedding geometry of numbers of gebits, but these gebits have a 'fuzzy' embedding in the base gebit. This is an indication of fuzzy homotopies (a homotopy is, put simply, an embedding of one space into another). Here we see the emergence of geometry, not only of space but also of the internal flavour symmetry spaces of quantum fields. 


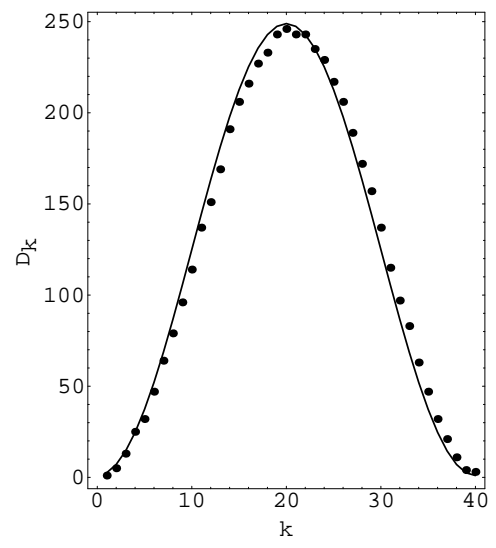

(a)

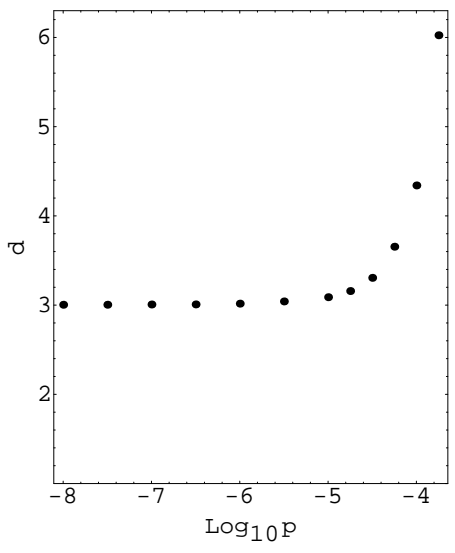

(b)

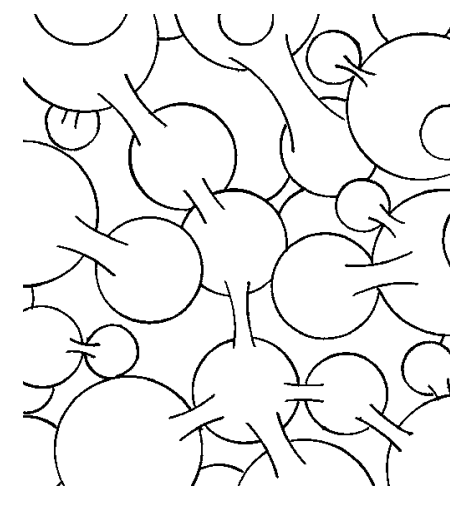

(c)

Figure 6: (a) Points show the $D_{k}$ set and $L=40$ value found by numerically maximising $\mathcal{P}[D, L, N]$ for $\log _{10} p=-6$ for fixed $N_{g}=5000$. Curve shows $D_{k} \propto \sin ^{d-1}\left(\frac{\pi k}{L}\right)$ with best fit $d=3.16$ and $L=40$, showing excellent agreement, and indicating embeddability in an $S^{3}$ with some topological defects. (b) Dimensionality $d$ of the gebits as a function of the probability $p$. (c) Graphical depiction of the 'process space' at one stage of the iterative process-time showing a quantum-foam structure formed from embeddings and links of the kind in (a). The linkage connections have the distribution of a 3D space, but the individual gebit components are closed compact spaces and cannot be embedded in a 3D background space. So the drawing is only suggestive. Nevertheless this figure indicates that process physics generates a cellular information system, where the behaviour is determined at all levels by internal information.

The base gebits $g_{1}, g_{2}, \ldots$ arising from the SRN together with their embedded topological defects have another remarkable property: they are 'sticky' with respect to the iterator. Consider the larger valued $B_{i j}$ within a given gebit $g$, they form tree graphs and most tree-graph adjacency matrices are singular $\left(\operatorname{det}\left(g_{\text {tree }}\right)=0\right)$. However the presence of other smaller valued $B_{i j}$ and the general background noise ensures that $\operatorname{det}(g)$ is small but not exactly zero. Then the $B$ matrix has an inverse with large components that act to cross-link the new and existing gebits. This cross-linking is itself random, due to the presence of background noise, and the above analysis may again be used and we would conclude that the base gebits themselves are formed into a 3D hypersphere with embedded topological defects. The nature of the resulting 3D process-space is suggestively indicated in Fig.6c, and behaves essentially as a quantum foam [36].

Over ongoing iterations the existing gebits become cross-linked and eventually lose their ability to undergo further linking; they lose their 'stickiness' and decay. The value 
of the parameter $\alpha$ in (2) must be small enough that the 'stickiness' persists over many iterations, that is, it is not quenched too quickly, otherwise the emergent network will not grow. Hence the emergent space is $3 \mathrm{D}$ but is continually undergoing replacement of its component gebits; it is an informational process-space, in sharp distinction to the non-process continuum geometrical spaces that have played a dominant role in modelling physical space. If the noise is 'turned off' then this emergent dissipative space will decay and cease to exist. We thus see that the nature of space is deeply related to the logic of the limitations of logic, as implemented here as a self-referentially limited neural network.

\section{$5 \quad$ Gebits and their Topological Defects}

\subsection{Gebits as Skyrmions}

We need to extract convenient but approximate syntactical descriptions of the semantic information in the network, and these will have the form of a sequence of mathematical constructions, the first being the Quantum Homotopic Field Theory. Importantly they must all retain explicit manifestations of the SRN. To this end first consider the special case of the iterator when the SRN is frozen at a particular $w$, that is we consider iterations with an artificially fixed SRN term. Then it may be shown that the iterator is equivalent to the minimisation of an 'energy' expression (remember that $B$ and $w$ are antisymmetric)

$$
E[B ; w]=-\frac{\alpha}{2} \operatorname{Tr}\left[B^{2}\right]-\alpha \operatorname{Tr} \operatorname{Ln}[B]+\operatorname{Tr}[w B] .
$$

Note that for disconnected gebits $g_{1}$ and $g_{2}$ this energy is additive, $E\left[g_{1} \oplus g_{2}\right]=E\left[g_{1}\right]+$ $E\left[g_{2}\right]$. Now suppose the fixed $w$ has the form of a gebit approximating an $S^{3}$ network with one embedded topological defect which is itself an $S^{3}$ network, for simplicity. So we are dissecting the gebit into base gebit, defect gebit and linkings or embeddings between the two. We also ignore the rest of the network, which is permissible if our gebit is disconnected from it. Now if $\operatorname{det}(w)$ is not small, then this gebit is non-sticky, and for small $\alpha$, the iterator converges to $B \approx \frac{1}{\alpha} w$, namely an enhancement only of the gebit. However because the gebits are rare constructs they tend to be composed of larger $w_{i j}$ forming tree structures, linked by smaller valued $w_{i j}$. The tree components make $\operatorname{det}(w)$ smaller, and then the inverse $B^{-1}$ is activated and generates new links. Hence, in particular, the topological defect relaxes, according to the 'energy' measure, with respect to the base gebit. This relaxation is an example of a 'non-linear elastic' process [37]. The above gebit has the form of a mapping $\pi: S \rightarrow \Sigma$ from a base space to a target space. Manton $[38,39,40]$ has constructed the continuum form for the 'elastic energy' of such an embedding and for $\pi: S^{3} \rightarrow S^{3}$ it is the Skyrme energy

$$
E[U]=\int\left[-\frac{1}{2} \operatorname{Tr}\left(\partial_{i} U U^{-1} \partial_{i} U U^{-1}\right)-\frac{1}{16} \operatorname{Tr}\left[\partial_{i} U U^{-1}, \partial_{i} U U^{-1}\right]^{2}\right]
$$


where $U(x)$ is an element of $S U(2)$. Via the parametrisation $U(x)=\sigma(x)+i \vec{\pi}(x) \cdot \vec{\tau}$, where the $\tau_{i}$ are Pauli matrices, we have $\sigma(x)^{2}+\vec{\pi}(x)^{2}=1$, which parametrises an $S^{3}$ as a unit hypersphere embedded in $E^{4}$ (which has no ontological significance, of course). Non-trivial minima of $E[U]$ are known as Skyrmions (a form of topological soliton), and have $Z= \pm 1, \pm 2, \ldots$, where $Z$ is the winding number of the map,

$$
Z=\frac{1}{24 \pi^{2}} \int \sum \epsilon_{i j k} \operatorname{Tr}\left(\partial_{i} U U^{-1} \partial_{j} U U^{-1} \partial_{k} U U^{-1}\right) .
$$

The first key to extracting emergent phenomena from the stochastic neural network is the validity of this continuum analogue, namely that $E[B ; w]$ and $E[U]$ are describing essentially the same 'energy' reduction process. This requires detailed analysis.

\subsection{Absence of a Cosmic Code}

This 'frozen' SRN analysis of course does not match the time-evolution of the full iterator (2), for this displays a much richer collection of processes. With ongoing new noise in each iteration and the saturation of the linkage possibilities of the gebits emerging from this noise, there arises a process of ongoing birth, linking and then decay of most patterns. The task is then to identify those particular patterns that survive this flux, even though all components of these patterns eventually disappear, and to attempt a description of their modes of behaviour. This brings out the very biological nature of the information processing in the SNN, and which appears to be characteristic of a 'pure' semantic information system. Kitto [18] has further investigated the analogies between process physics and living systems. The emergent 'laws of physics' are the habitual habits of this system, and it appears that they may be identified. However there is no encoding mechanism for these 'laws', they are continually manifested; there is no cosmic code. In contrast living or biological systems could be defined as those emergent patterns which discovered how to encode their 'laws' in a syntactical genetic code. Nevertheless such biological systems make extensive use of semantic information at all levels as their genetic code is expressed in the phenotype.

\subsection{Entrapped Topological Defects}

In general each gebit, as it emerges from the SRN, has active nodes and embedded topological defects, again with active nodes. Further there will be defects embedded in the defects and so on, and so gebits begin to have the appearance of a fractal defect structure, and with all the defects having various classifications and associated winding numbers. The energy analogy above suggests that defects with opposite winding numbers at the same fractal depth may annihilate by drifting together and merging. Furthermore 
the embedding of the defects is unlikely to be 'classical', in the sense of being described by a mapping $\pi(x)$, but rather would be fuzzy, i.e described by some functional, $F[\pi]$, which would correspond to a classical embedding only if $F$ has a very sharp supremum at one particular $\pi=\pi_{c l}$. As well these gebits are undergoing linking because their active nodes (see [9] for more discussion) activate the $B^{-1}$ new-links process between them, and so by analogy the gebits themselves form larger structures with embedded fuzzy topological defects. This emergent behaviour is suggestive of a quantum space foam, but one containing topological defects which will be preserved by the system, unless annihilation events occur. If these topological defects are sufficiently rich in fractal structure so as to be preserved, then their initial formation would have occurred as the process-space relaxed out of its initial essentially random form. This phase would correspond to the early stages of the Big-Bang. Once the topological defects are trapped in the process-space they are doomed to meander through that space by essentially self-replicating, i.e. continually having their components die away and be replaced by similar components. These residual topological defects are what we call matter. The behaviour of both the process-space and its defects is clearly determined by the same network processes; we have an essential unification of space and matter phenomena. This emergent quantum foam-like behaviour suggests that the full generic description of the network behaviour is via the Quantum Homotopic Field Theory (QHFT) of the next section. We also see that cellular structures are a general feature of semantic information systems, with the information necessarily distributed.

\section{Quantum Homotopic Field Theory}

\subsection{Functional Schrödinger Equation}

Because of the iterator the resource is the large valued $B_{i j}$ from the SRN because they form the 'sticky' gebits which are self-assembled into the non-flat compact 3D processspace. The accompanying topological defects within these gebits and also the topological defects within the process space require a more subtle description. The key behavioural mode for those defects which are sufficiently large (with respect to the number of component gebits) is that their existence, as identified by their topological properties, will survive the ongoing process of mutation, decay and regeneration; they are topologically self-replicating. Consider the analogy of a closed loop of string containing a knot - if, as the string ages, we replace small sections of the string by new pieces then eventually all of the string will be replaced; however the relational information represented by the knot will remain unaffected as only the topology of the knot is preserved. In the process-space there will be gebits embedded in gebits, and so forth, in topologically non-trivial ways; 
the topology of these embeddings is all that will be self-replicated in the processing of the dissipative structure.

To analyse and model the 'life' of these topological defects we need to characterise their general behaviour: if sufficiently large (i) they will self-replicate if topological nontrivial, (ii) we may apply continuum homotopy theory to tell us which embeddings are topologically non-trivial, (iii) defects will only dissipate if embeddings of 'opposite winding number' (these classify the topology of the embedding) engage one another, (iv) the embeddings will be in general fractal, and (iv) the embeddings need not be 'classical', i.e., the embeddings will be fuzzy. Tracking the coarse-grained behaviour of such a system led to the development of a new form of quantum field theory: Quantum Homotopy Field Theory (QHFT). This models both the process-space and the topological defects.

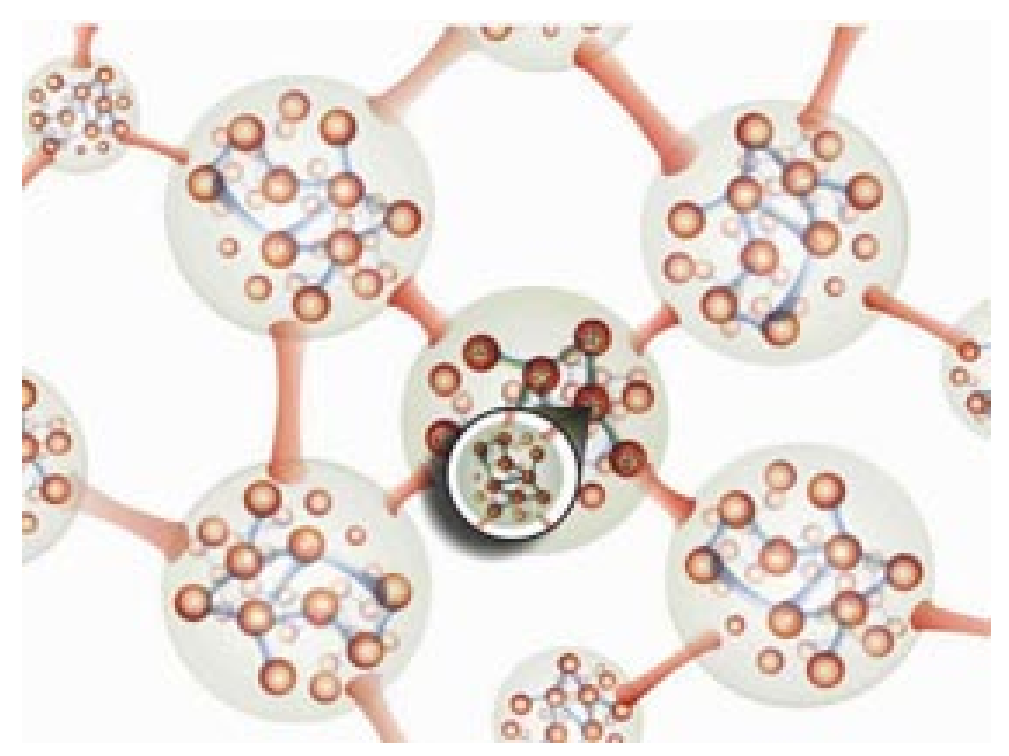

Figure 7: An artistic representation of the functional $\Psi[\{\pi\} ; t]$ showing dominant homotopies. The 'magnifying glass' indicates that these mappings can be nested. Graphic by C. Klinger. First published as the cover graphic of [11].

To construct this QHFT we introduce an appropriate configuration space, namely all the possible homotopic mappings $\pi_{\alpha \beta}: S_{\beta} \rightarrow S_{\alpha}$, where the $S_{1}, S_{2}, .$. , describing 'clean' or topological-defect free gebits, are compact spaces of various types. Then QHFT has the form of an iterative functional Schrödinger equation for the discrete time-evolution of a wave-functional $\Psi\left[\ldots, \pi_{\alpha \beta}, \ldots . ; t\right]$

$$
\Psi\left[\ldots, \pi_{\alpha \beta}, \ldots ; t+\Delta t\right]=\Psi\left[\ldots, \pi_{\alpha \beta}, \ldots ; t\right]-i H \Psi\left[\ldots, \pi_{\alpha \beta}, \ldots ; t\right] \Delta t+\text { QSD terms. }
$$

This form arises as it is models the preservation of semantic information, by means of a unitary time evolution, even in the presence of the noise in the Quantum State Diffusion 
(QSD) terms. Because of the QSD noise (17) is an irreversible quantum system. The time step $\Delta t$ in (17) is relative to the scale of the fractal processes being explicitly described, as we are using a configuration space of mappings between prescribed gebits. At smaller scales we would need a smaller value for $\Delta t$. Clearly this invokes a (finite) renormalisation scheme. We now discuss the form of the hamiltonian and the QSD terms.

First (17), without the QSD term, has a form analogous to a 'third quantised' system, in conventional terminology [41]. These systems were considered as perhaps capable of generating a quantum theory of gravity. The argument here is that this is the emergent behaviour of the SNN, and it does indeed lead to quantum gravity, but with quantum matter as well. More importantly we understand the origin of (17), and it will lead to quantum and then classical gravity, rather than arise from classical gravity via some ad hoc or heuristic quantisation procedure.

Depending on the 'peaks' of $\Psi$ and the connectivity of the resultant dominant mappings such mappings are to be interpreted as either embeddings or links; Fig.7 then suggests the dominant process-space form within $\Psi$ showing both links and embeddings. The emergent process-space then has the characteristics of a quantum foam. Note that, as indicated in Fig.7, the original start-up links and nodes are now absent. Contrary to the suggestion in Fig.7, this process space cannot be embedded in a finite dimensional geometric space with the emergent metric preserved, as it is composed of nested finite-dimensional closed spaces.

\subsection{Homotopy Hamiltonian}

We now consider the form of the hamiltonian $H$. The previous section suggested that Manton's non-linear elasticity interpretation of the Skyrme energy is appropriate to the $\mathrm{SNN}$. This then suggests that $\mathrm{H}$ is the functional operator

$$
H=\sum_{\alpha \neq \beta} h\left[\frac{\delta}{\delta \pi_{\alpha \beta}}, \pi_{\alpha \beta}\right]
$$

where $h\left[\frac{\delta}{\delta \pi}, \pi\right]$ is the (quantum) Skyrme Hamiltonian functional operator for the system based on making fuzzy the mappings $\pi: S \rightarrow \Sigma$, by having $h$ act on wave-functionals of the form $\Psi[\pi(x) ; t]$. Then $H$ is the sum of pairwise embedding or homotopy hamiltonians. The corresponding functional Schrödinger equation would simply describe the time evolution of quantised Skyrmions with the base space fixed, and $\Sigma \in S U(2)$. There have been very few analyses of this class of problem, and then the base space is usually taken to be $E^{3}$. We shall not give the explicit form of $h$ as it is complicated, but wait to present the associated action.

In the absence of the QSD terms the time evolution in (17) can be formally written 
as a functional integral

$$
\Psi\left[\{\pi\} ; t^{\prime}\right]=\int \prod_{\alpha \neq \beta} \mathcal{D} \tilde{\pi}_{\alpha \beta} e^{i S[\{\tilde{\pi}\}]} \Psi[\{\pi\} ; t],
$$

where, using the continuum $t$ limit notation, the action is a sum of pairwise actions,

$$
\begin{gathered}
S[\{\tilde{\pi}\}]=\sum_{\alpha \neq \beta} S_{\alpha \beta}\left[\tilde{\pi}_{\alpha \beta}\right] \\
S_{\alpha \beta}[\tilde{\pi}]=\int_{t}^{t^{\prime}} d t^{\prime \prime} \int d^{n} x \sqrt{-g}\left[\frac{1}{2} \operatorname{Tr}\left(\partial_{\mu} \tilde{U} \tilde{U}^{-1} \partial^{\mu} \tilde{U} \tilde{U}^{-1}\right)+\frac{1}{16} \operatorname{Tr}\left[\partial_{\mu} \tilde{U} \tilde{U}^{-1}, \partial^{\nu} \tilde{U} \tilde{U}^{-1}\right]^{2}\right],
\end{gathered}
$$

and the now time-dependent (indicated by the tilde symbol) mappings $\tilde{\pi}$ are parametrised by $\tilde{U}(x, t), \tilde{U} \in S_{\alpha}$. The metric $g_{\mu \nu}$ is that of the $n$-dimensional base space, $S_{\beta}$, in $\pi_{\alpha, \beta}: S_{\beta} \rightarrow S_{\alpha}$. As usual in the functional integral formalism the functional derivatives in the quantum hamiltonian, in (18), now manifest as the time components $\partial_{0}$ in (21), so now (21) has the form of a 'classical' action, and we see the emergence of 'classical' fields, though the emergence of 'classical' behaviour is a more complex process. Eqns.(17) or (19) describe an infinite set of quantum skyrme systems, coupled in a pairwise manner. Note that each homotopic mapping appears in both orders; namely $\pi_{\alpha \beta}$ and $\pi_{\beta \alpha}$.

\subsection{Quantum State Diffusion}

The Quantum State Diffusion (QSD) [42] terms are non-linear and stochastic,

$$
\text { QSD terms }=\sum_{\gamma}\left(<L_{\gamma}^{\dagger}>L_{\gamma}-\frac{1}{2} L_{\gamma}^{\dagger} L_{\gamma}-<L_{\gamma}^{\dagger}><L_{\gamma}>\right) \Psi \Delta t+\sum_{\gamma}\left(L_{\gamma}-<L_{\gamma}>\right) \Psi \Delta \xi_{\gamma}
$$

which involves summation over the class of Linblad functional operators $L_{\gamma}$. The QSD terms are up to 5 th order in $\Psi$, as in general

$$
<A>_{t} \equiv \int \prod_{\alpha \neq \beta} \mathcal{D} \pi_{\alpha \beta} \Psi[\{\pi\} ; t]^{*} A \Psi[\{\pi\} ; t]
$$

and where $\Delta \xi_{\gamma}$ are complex statistical variables with means $M\left(\Delta \xi_{\gamma}\right)=0, M\left(\Delta \xi_{\gamma} \Delta \xi_{\gamma^{\prime}}\right)=$ 0 and $M\left(\Delta \xi_{\gamma}^{*} \Delta \xi_{\gamma^{\prime}}\right)=\delta\left(\gamma-\gamma^{\prime}\right) \Delta t$. The remarkable property of this QSD term is that the unitarity of the time evolution in (17) is maintained.

\subsection{Emergent Classicality}

These QSD terms are ultimately responsible for the emergence of classicality via an objectification process [42], but in particular they produce wave-function(al) collapses 
during quantum measurements, as the QSD terms tend to 'sharpen' the fuzzy homotopies towards classical or sharp homotopies (the forms of the Linblads will be discussed in detail elsewhere). So the QSD terms, as residual SRN effects, lead to the Born quantum measurement random behaviour, but here arising from the process physics, and not being invoked as a metarule. Keeping the QSD terms leads to a functional integral representation for a density matrix formalism in place of (19), and this amounts to a derivation of the decoherence formalism which is usually arrived at by invoking the Born measurement metarule. Here we see that 'decoherence' arises from the limitations on self-referencing.

In the above we have a deterministic and unitary evolution, tracking and preserving topologically encoded information, together with the stochastic QSD terms, whose form protects that information during localisation events, and which also ensures the full matching in QHFT of process-time to real time: an ordering of events, an intrinsic direction or 'arrow' of time and a modelling of the contingent present moment effect. So we see that process physics generates a complete theory of quantum measurements involving the non-local, non-linear and stochastic QSD terms. It does this because it generates both the 'objectification' process associated with the classical apparatus and the actual process of (partial) wavefunctional collapse as the quantum modes interact with the measuring apparatus. Indeed many of the mysteries of quantum measurement are resolved when it is realised that it is the measuring apparatus itself that actively provokes the collapse, and it does so because the QSD process is most active when the system deviates strongly from its dominant mode, namely the ongoing relaxation of the system to a 3D process-space, and matter survives only because of its topological form. This is essentially the process that Penrose [43] suggested, namely that the quantum measurement process is essentially a manifestation of quantum gravity. The demonstration of the validity of the Penrose argument of course could only come about when quantum gravity was derived from deeper considerations, and not by some ad hoc argument such as the quantisation of Einstein's classical spacetime model.

That the non-local QSD terms lead to wavefunctional collpase is relevant to the new theory of gravity in Sect.9.5. This collapse amounts to an ongoing sharpening of the homotopic mappings towards a 'classical' 3D configuration - resulting in essentially the process we have long recognised as 'space'. Being non-local the collapse process does not involve any propagation effects, that is the collapse does not require any effect to propagate through the space. For that reason the self-generation of space is in some sense action at a distance, and the emergence of such a quantum process underlying reality is, of course, contrary to the long-held belief by physicists that such action is unacceptable, though that belief arose before the quantum collapse was experimentally shown to display action at a distance in the Aspect experiment. Hence we begin to appreciate why the 
new theory of gravity does not involve the maximum speed $c$ of propagation through space, and why it does not predict the GR gravitational waves travelling at speed $c$, of the kind long searched for but not detected.

The mappings $\pi_{\alpha \beta}$ are related to group manifold parameter spaces with the group determined by the dynamical stability of the mappings. This symmetry leads to the flavour symmetry of the standard model. Quantum homotopic mappings or skyrmions behave as fermionic or bosonic modes for appropriate winding numbers; so process physics predicts both fermionic and bosonic quantum modes, but with these associated with topologically encoded information and not with objects or 'particles'.

\section{$7 \quad$ Emergent Quantum Field Theory of Matter}

\subsection{Quantum Field Theory}

The QHFT is a very complex 'book-keeping' system for the emergent properties of the neural network, and we now sketch how we may extract a more familiar Quantum Field Theory (QFT) that relates to the standard model of 'particle' physics. An effective QFT should reproduce the emergence of the process-space part of the quantum foam, particularly its 3D aspects. The QSD processes play a key role in this as they tend to enhance classicality. Hence at an appropriate scale QHFT should approximate to a more conventional QFT, namely the emergence of a wave-functional system $\Psi[U(x) ; t]$ where the configuration space is that of homotopies from a 3 -space to $U(x) \in G$, where $G$ is some group manifold space. This $G$ describes 'flavour' degrees of freedom. So we are coarse-graining out the gebit structure of the quantum-foam. Hence the Schrödinger wavefunctional equation for this QFT will have the form

$$
\Psi[U ; t+\Delta t]=\Psi[U ; t]-i H \Psi[U ; t] \Delta t+\mathrm{QSD} \text { terms }
$$

where the general form of $H$ is known, and where a new residual manifestation of the SRN appears as the new QSD terms. This system describes skyrmions embedded in a continuum space. It is significant that such Skyrmions are only stable, at least in flat space and for static skyrmions, if that space is 3D. This tends to confirm the observation that 3D space is special for the neural network process system.

\subsection{Emergent Flavour and Hidden Colour}

Again, in the absence of the QSD terms, we may express (24) in terms of the functional integral

$$
\Psi\left[U ; t^{\prime}\right]=\int \mathcal{D} \tilde{U} e^{i S[\tilde{U}]} \Psi[U ; t]
$$


To gain some insight into the phenomena present in (24) or (25), it is convenient to use the fact that functional integrals of this Skyrmionic form my be written in terms of Grassmann-variable functional integrals, but only by introducing a fictitious 'metacolour' degree of freedom and associated coloured fictitious vector bosons. This is essentially the reverse of the Functional Integral Calculus (FIC) hadronisation technique in the Global Colour Model (GCM) of QCD [30]. The action for the Grassmann and vector boson part of the system is of the form (written for flat space)

$$
S\left[\bar{p}, p, A_{\mu}^{a}\right]=\int d^{4} x\left(\bar{p} \gamma^{\mu}\left(i \partial_{\mu}+g \frac{\lambda^{a}}{2} A_{\mu}^{a}\right) p-\frac{1}{4} F_{\mu \nu}^{a}(A) F^{a \mu \nu}(A)\right),
$$

where the Grassmann variables $p_{f c}(x)$ and $\bar{p}_{f c}(x)$ have flavour and metacolour labels. The Skyrmions are then re-constructed, in this system, as topological solitons formed from the low energy Nambu-Goldstone modes; other emergent modes are of higher energy and can be ignored. These coloured and flavoured but fictitious fermionic fields $\bar{p}$ and $p$ correspond to the proposed preon system $[44,45]$. As they are purely fictitious, in the sense that there are no excitations in the system corresponding to them, the metacolour degree of freedom must be hidden or confined. We thus arrive at the general feature of the standard model of particles with flavour and confined colour degrees of freedom. Then while the QHFT and the QFT represent an induced syntax for the semantic information, the preons may be considered as an induced 'alphabet' for that syntax. The advantage of introducing this preon alphabet is that we can more easily determine the states of the system by using the more familiar language of fermions and bosons, rather than working with the skyrmionic system, so long as only colour singlet states are finally permitted. In order to establish fermionic behaviour a Wess-Zumino (WZ) process must be extracted from the iterator behaviour or the QHFT. Such a WZ process is time-dependent, and so cannot arise from the frozen SRN argument in Sect.4. It is important to note that (26) and the action in (25) are certainly not the final forms. Further analysis will be required to fully extract the induced actions for the emergent QFT.

\section{Emergent Universe}

\subsection{Multi-World Universe and the 'Dark Energy' Effect}

Process physics predicts that the neural network behaviour will be characterised by a growing 3-dimensional process-space having, at a large scale, the form of a $S^{3}$ hypersphere. It is possible to give the dominant rate of growth of this hypersphere. However first, from random graph theory [46], we expect more than one such spatial system, with each having the character of a growing hypersphere, and all embedded in the random background discussed previously. This background has no metric structure, and so these 
various hyperspheres have no distance measure over them. We have then a multi-world universe (our 'universe' being merely one of these 'worlds'). Being process spaces they compete for new gebits, and so long as we avoid a saturation case, each will grow according to

$$
\frac{d N_{i}}{d t}=a N_{i}-b N_{i} \quad a>0, b>0,
$$

where the last term describes the decay of gebits at a rate $b$, while the first describes growth of the $i^{\text {th }}$ 'world', this being proportional to the size (as measured by its gebit content number) $N_{i}(t)$, as success in randomly attaching new gebits is proportional to the number of gebits present (the 'stickiness' effect), so long as we ignore the topological defects (quantum 'matter') as these have a different stickiness, and also affect the decay rate, and so slow down the expansion. Thus $N_{i}(t)$ will show exponential growth, as appears to be the case as indicated by recent observations of very distant supernovae counts [47]. Hence process physics predicts a positive cosmological constant, now called the 'dark energy' effect, and that this is unrelated to the phenomenon of gravity. Indeed this multi-world model is incompatible with general relativity, as it is not capable of even describing the non-geometric background or embedding system. In this enlarged cosmology each world would have its own independent big-bang beginning, but then it is no longer necessary for this on-going ensemble of worlds to have had a beginning itself, as we may presumably take the system start-up time to $-\infty$. Hence the observation of the cosmological constant effect is here to be interpreted as arising from the existence of these other worlds.

\subsection{Gravity and the Equivalence Principle}

One striking outcome of process physics is an explanation for the phenomenon of gravity. First note that matter is merely topological defects embedded in the process space, and we expect such defects to have a larger than usual gebit connectivity; indeed matter is a violation of the 3-D connectivity of this space, and it is for this reason we needed to introduce fields to emulate this extra non-spatial connectivity. One consequence of this is that in the region of these matter fields the gebits decay faster, they are less sticky because of the extra connectivity. Hence in this region, compared to other nearby matter-free regions the gebits are being 'turned over' more frequently but at the same time are less effective in attracting new gebits. Overall this suggests that matter-occupying-regions act as net sinks for gebits, and there will be a trend for the neighbouring quantum foam to undergo a diffusion/relaxation process in which the foam effectively moves towards the matter: matter acts as a sink for space, but never as a source. Such a process would clearly correspond to gravity. As the effect is described by a net diffusion/relaxation of space which acts equally on all surrounding matter, the in-fall mechanism is independent 
of the nature of the surrounding matter. This is nothing more than the Equivalence Principle, first noticed by Galileo. As well if the in-fall rate exceeds the rate at which 'motion' through the process-space is possible then an event horizon appears, and this is clearly the black hole scenario. Presumably at the core of a black-hole is a tangle of topological defects, so that the effective dimensionality is very high, and which maintains the in-flow of quantum foam. This in-flow is a loss of information, and so gravity itself is an information-loss process.

\subsection{Inertia}

One longstanding unsolved problem in physics is the explanation for inertia. This is the effect where objects continue in uniform motion unless acted upon by 'forces', and was first analysed by Galileo in the modern era, but of course Zeno made an early attempt. However there has never been an explanation for this effect. In Newtonian physics it was built into the syntactical description rather than being a prediction of that modelling. Of course current physics is essentially a static modelling of reality, with motion indirectly accessed via the geometrical-time metarule, and so the failure to explain motion is not unexpected. However process physics offers a simple explanation.

The argument for inertia follows from a simple self-consistency argument. Suppose a topological defect, or indeed a vast bound collection of such defects, is indeed 'in motion'. This implies that the gebits are being preferentially replaced in the direction of that 'motion', for motion as such is a self-replication process; there is no mechanism in process physics for a fixed pattern to say 'slide' through the quantum-foam. Rather motion is self-replication of the gebit connectivity patterns in a set direction. Since the newest gebits, and hence the stickiest gebits, in each topological defect, are on the side corresponding to the direction of motion, the gebits on that side are preferentially replaced. Hence the motion is self-consistent and self-perpetuating.

An additional effect expected in process physics is that such motion results in a time dilation and length contraction effects; the self-replication effect is to be considered as partly the self-replication associated with any internal oscillations and partly self-replication associated with 'motion'. This 'competition for resources' results in the slowing down of internal oscillations, an idea discussed by Toffoli [48]. Such effects have been seen in a variety of 'non-relativistic' continuum systems [49], and indeed they have a long history. In particular emergent Lorentz relativistic effects have been seen in the modelling of dislocations in crystals [50] where 'breather modes' as solitons arise. Hence the lesson is that emergent Lorentz relativistic effects are generic whenever there is a unification of the substratum system and embedded excitations, here the solitons are dynamical emergent features within some dynamical background, rather than being merely 
'pasted' onto some a priori geometrised and so structureless background. Bell [52] has argued for this dynamical interpretation of Lorentz symmetry, as indeed did Lorentz, until this view was overwhelmed by the Einstein spacetime formalisation of relativistic effects. This is discussed in more detail in the next section. More recently similar ideas have emerged [51] in the analysis of the sound waves in classical fluids. In later sections we assume that the QHFT will also display these generic Lorentzian dynamical effects, namely the time-dilation/length-contraction effects.

Geometry is clearly an emergent but approximate phenomenological language; it is certainly not fundamental, and the implicit assumption in physics that it is fundamental has caused many problems and confusions, in particular the absence of 'structure', and the effect of that structure on 'objects' moving through that structure. In process physics the quantum-foam represents an actual spatial system, and as such amounts to a preferred frame of reference, but one, as we shall see, that is well hidden by the timedilation and length-contraction effects. Later we show that contrary to popular notions the Michelson interferometer, as one example, can reveal this frame when operated in gas mode, and the data from the 1887 Michelson-Morley and the 1925/26 Miller interferometer experiments is analysed. These data show that evidence of absolute motion relative to this quantum-foam space has been present in the non-vacuum interferometer data for over 100 years. However we shall see that the cosmic speed of the solar system through the quantum foam, though comparable to the speed determined from the dipole-fit to the Cosmic Microwave Background (CMB) radiation, is in quite a different direction. The explanation for this is obvious once we consider in more detail the in-flow explanation of gravity. As well the orbital velocity of the earth about the sun is revealed as well as the quantum-foam in-flow towards the sun, as described above.

\section{Quantum Foam Flow and a New Theory of Gravity}

We begin here the modelling of the quantum-foam system that is space and of the motion of 'objects' through that space. Gravity is caused by the inhomogeneous flow of the quantum foam towards matter. Motion relative to the quantum foam, that is absolute motion, has been repeatedly detected in Michelson interferometer experiments and others for over 100 years, though it was only in 2002 that a key aspect of the physics of the interferometer was discovered. The gravitational in-flow is also revealed by analysis of old interferometer experimental data. So far there is no derivation of the flow physics for this quantum foam system from the fundamental QHFT, and so a 'classical' flow equation will be suggested based upon arguments presented in this section. It will be 
seen that features of this flow equation such as turbulence are observable and already apparent in existing experimental data. A detailed study of this turbulence will provide a means to access and check the underlying flow equation, particularly as new 1st-order interferometer designs will make observation of absolute motion effects particularly easy. This new theory of gravity is different in its predictions to both the Newtonian theory and to the Einsteinian General Relativity theory, and such differences are easily detectable.

\subsection{Classical Effects of Quantum Foam}

We begin here the analysis that will lead to the new theory and explanation of gravity. In this theory gravitational effects are caused solely by an inhomogeneous flow of the quantum foam. Essentially matter effectively acts as a 'sink' for that quantum foam. To begin with it should be noted that even Newtonian gravity is suggestive of a flow explanation of gravity. In that theory the gravitational acceleration $\mathbf{g}$ is determined by the matter density $\rho$ according to

$$
\nabla \cdot \mathbf{g}=-4 \pi G \rho
$$

For $\nabla \times \mathbf{g}=0$ this gravitational acceleration $\mathbf{g}$ may be written as the gradient of the gravitational potential $\Phi$

$$
\mathrm{g}=-\nabla \Phi
$$

where the gravitational potential is now determined by $\nabla^{2} \Phi=4 \pi G \rho$. Here, as usual, $G$ is the gravitational constant. Now as $\rho \geq 0$ we can choose to have $\Phi \leq 0$ everywhere if $\Phi \rightarrow 0$ at infinity. So we can introduce $\mathbf{v}^{2}=-2 \Phi \geq 0$ where $\mathbf{v}$ is some velocity vector field. Here the value of $\mathbf{v}^{2}$ is specified, but not the direction of $\mathbf{v}$. Then

$$
\mathbf{g}=\frac{1}{2} \nabla\left(\mathbf{v}^{2}\right)=(\mathbf{v} \cdot \nabla) \mathbf{v}+\mathbf{v} \times(\nabla \times \mathbf{v}) .
$$

For irrotational flow $\nabla \times \mathbf{v}=\mathbf{0}$. Then $\mathbf{g}$ is the usual Euler expression for the acceleration of a fluid element in a time-independent or stationary fluid flow. If the flow is time dependent that expression is expected to become

$$
\mathbf{g}=(\mathbf{v} \cdot \nabla) \mathbf{v}+\mathbf{v} \times(\nabla \times \mathbf{v})+\frac{\partial \mathbf{v}}{\partial t} .
$$

This equation is then to be accompanied by the 'Newtonian equation' for the flow field

$$
\frac{1}{2} \nabla^{2}\left(\mathbf{v}^{2}\right)=-4 \pi G \rho
$$

To be consistent with (28) in the case of a time-dependent matter density this equation should be generalised to

$$
\frac{\partial}{\partial t}(\nabla \cdot \mathbf{v})+\frac{1}{2} \nabla^{2}\left(\mathbf{v}^{2}\right)=-4 \pi G \rho .
$$


This hints at a fluid flow interpretation of Newtonian gravity Of course within the fluid flow interpretation (31) and (33) are together equivalent to the Universal Inverse Square Law for Gravity. Indeed for a spherically symmetric distribution of matter of total mass $M$ the velocity field outside of the matter

$$
\mathbf{v}(\mathbf{r})=-\sqrt{\frac{2 G M}{r}} \hat{\mathbf{r}},
$$

satisfies (33) and reproduces the inverse square law form for $\mathbf{g}$ using (31):

$$
\mathbf{g}=-\frac{G M}{r^{2}} \hat{\mathbf{r}}
$$

The in-flow direction $-\hat{\mathbf{r}}$ in (34) may be replaced by any other direction, in which case however the direction of $\mathbf{g}$ in (35) remains radial.

Of the many new effects predicted by the generalisation of (33) one is that this 'Inverse Square Law' is only valid outside of spherically symmetric matter systems. Then, for example, the 'Inverse Square Law' is expected to be inapplicable to spiral galaxies. The incorrect assumption of the universal validity of this law led to the notion of 'dark matter' in order to reconcile the faster observed rotation velocities of matter within such galaxies compared to that predicted by the above law.

To arrive at the new in-flow theory of gravity we require that the velocity field $\mathbf{v}(\mathbf{r}, t)$ be specified and measurable with respect to a suitable frame of reference. We shall use the Cosmic Microwave Background (CMB) frame of reference for that purpose. Then an 'object' has velocity $\mathbf{v}_{0}(t)=d \mathbf{r}_{0}(t) / d t$ with respect to that CMB frame, where $\mathbf{r}_{0}(t)$ is the position of the object with respect to that frame. We then define

$$
\mathbf{v}_{R}(t)=\mathbf{v}_{0}(t)-\mathbf{v}\left(\mathbf{r}_{0}(t), t\right)
$$

as the velocity of the object relative to the quantum foam at the location of the object.

Process Physics appears to be leading to the Lorentzian interpretation of so called 'relativistic effects'. This means that the speed of light is only 'c' wrt the quantumfoam system, and that time dilation effects for clocks and length contraction effects for rods are caused by the motion of clocks and rods relative to the quantum foam. So these effects are real dynamical effects caused by the quantum foam, and are not to be interpreted as spacetime effects as suggested by Einstein. To arrive at the dynamical description of the various effects of the quantum foam we shall introduce conjectures that essentially lead to a phenomenological description of these effects. In the future we expect to be able to derive this dynamics directly from the QHFT formalism. First we shall conjecture that the path of an object through an inhomogeneous and time-varying quantum-foam is determined by a variational principle, namely the path $\mathbf{r}_{0}(t)$ minimises 
the travel time (for early investigations of the in-flow approach to gravity see Ives[53] and Kirkwood[54, 55]),

$$
\tau\left[\mathbf{r}_{0}\right]=\int d t\left(1-\frac{\mathbf{v}_{R}^{2}}{c^{2}}\right)^{1 / 2},
$$

with $\mathbf{v}_{R}$ given by (36). Under a deformation of the trajectory $\mathbf{r}_{0}(t) \rightarrow \mathbf{r}_{0}(t)+\delta \mathbf{r}_{0}(t)$, $\mathbf{v}_{0}(t) \rightarrow \mathbf{v}_{0}(t)+\frac{d \delta \mathbf{r}_{0}(t)}{d t}$, and we also have

$$
\mathbf{v}\left(\mathbf{r}_{0}(t)+\delta \mathbf{r}_{0}(t), t\right)=\mathbf{v}\left(\mathbf{r}_{0}(t), t\right)+\left(\delta \mathbf{r}_{0}(t) . \nabla\right) \mathbf{v}\left(\mathbf{r}_{0}(t)\right)+\ldots
$$

Then

$$
\begin{aligned}
& \delta \tau=\tau\left[\mathbf{r}_{0}+\delta \mathbf{r}_{0}\right]-\tau\left[\mathbf{r}_{0}\right] \\
& =-\int d t \frac{1}{c^{2}} \mathbf{v}_{R} \cdot \delta \mathbf{v}_{R}\left(1-\frac{\mathbf{v}_{R}^{2}}{c^{2}}\right)^{-1 / 2}+\ldots \\
& =\int d t \frac{1}{c^{2}}\left(\mathbf{v}_{R} \cdot\left(\delta \mathbf{r}_{0} \cdot \nabla\right) \mathbf{v}-\mathbf{v}_{R} \cdot \frac{d\left(\delta \mathbf{r}_{0}\right)}{d t}\right)\left(1-\frac{\mathbf{v}_{R}^{2}}{c^{2}}\right)^{-1 / 2}+\ldots \\
& =\int d t \frac{1}{c^{2}}\left(\frac{\mathbf{v}_{R} \cdot\left(\delta \mathbf{r}_{0} \cdot \nabla\right) \mathbf{v}}{\sqrt{1-\frac{\mathbf{v}_{R}^{2}}{c^{2}}}}+\delta \mathbf{r}_{0} \cdot \frac{d}{d t} \frac{\mathbf{v}_{R}}{\sqrt{1-\frac{\mathbf{v}_{R}^{2}}{c^{2}}}}\right)+\ldots \\
& =\int d t \frac{1}{c^{2}} \delta \mathbf{r}_{0} \cdot\left(\frac{\left(\mathbf{v}_{R} \cdot \nabla\right) \mathbf{v}+\mathbf{v}_{R} \times(\nabla \times \mathbf{v})}{\sqrt{1-\frac{\mathbf{v}_{R}^{2}}{c^{2}}}}+\frac{d}{d t} \frac{\mathbf{v}_{R}}{\sqrt{1-\frac{\mathbf{v}_{R}^{2}}{c^{2}}}}\right)+\ldots
\end{aligned}
$$

Hence a trajectory $\mathbf{r}_{0}(t)$ determined by $\delta \tau=0$ to $O\left(\delta \mathbf{r}_{0}(t)^{2}\right)$ satisfies

$$
\frac{d}{d t} \frac{\mathbf{v}_{R}}{\sqrt{1-\frac{\mathbf{v}_{R}^{2}}{c^{2}}}}=-\frac{\left(\mathbf{v}_{R} \cdot \nabla\right) \mathbf{v}+\mathbf{v}_{R} \times(\nabla \times \mathbf{v})}{\sqrt{1-\frac{\mathbf{v}_{R}^{2}}{c^{2}}}} .
$$

Let us now write this in a more explicit form. This will also allow the low speed limit to be identified. Substituting $\mathbf{v}_{R}(t)=\mathbf{v}_{0}(t)-\mathbf{v}\left(\mathbf{r}_{0}(t), t\right)$ and using

$$
\frac{d \mathbf{v}\left(\mathbf{r}_{0}(t), t\right)}{d t}=\left(\mathbf{v}_{0} \cdot \nabla\right) \mathbf{v}+\frac{\partial \mathbf{v}}{\partial t}
$$

we obtain

$$
\frac{d}{d t} \frac{\mathbf{v}_{0}}{\sqrt{1-\frac{\mathbf{v}_{R}^{2}}{c^{2}}}}=\mathbf{v} \frac{d}{d t} \frac{1}{\sqrt{1-\frac{\mathbf{v}_{R}^{2}}{c^{2}}}}+\frac{(\mathbf{v} \cdot \nabla) \mathbf{v}-\mathbf{v}_{R} \times(\nabla \times \mathbf{v})+\frac{\partial \mathbf{v}}{\partial t}}{\sqrt{1-\frac{\mathbf{v}_{R}^{2}}{c^{2}}}}
$$


Then in the low speed limit $v_{R} \ll c$ we obtain

$$
\frac{d \mathbf{v}_{0}}{d t}=(\mathbf{v} \cdot \nabla) \mathbf{v}-\mathbf{v}_{R} \times(\nabla \times \mathbf{v})+\frac{\partial \mathbf{v}}{\partial t}=\mathbf{g}\left(\mathbf{r}_{0}(t), t\right)+(\nabla \times \mathbf{v}) \times \mathbf{v}_{0},
$$

which agrees with the 'Newtonian' form (31) for zero vorticity $(\nabla \times \mathbf{v}=0)$. Hence (42) is a generalisation of (31) to include Lorentzian dynamical effects, for in (42) we can multiply both sides by the rest mass $m_{0}$ of the object, and then (42) involves

$$
m\left(\mathbf{v}_{R}\right)=\frac{m_{0}}{\sqrt{1-\frac{\mathbf{v}_{R}^{2}}{c^{2}}}},
$$

the so called 'relativistic' mass, and (42) acquires the form $\frac{d}{d t}\left(m\left(\mathbf{v}_{R}\right) \mathbf{v}_{0}\right)=\mathbf{F}$, where $\mathbf{F}$ is an effective 'force' caused by the inhomogeneities and time-variation of the flow. This is essentially Newton's 2nd Law of Motion in the case of gravity only. That $m_{0}$ cancels is the equivalence principle, and which acquires a simple explanation in terms of the flow. Note that the occurrence of $1 / \sqrt{1-\frac{\mathbf{v}_{R}^{2}}{c^{2}}}$ will lead to the precession of the perihelion of planetary orbits, and also to horizon effects wherever $|\mathbf{v}|=c$ : the region where $|\mathbf{v}|<c$ is inaccessible from the region where $|\mathbf{v}|>c$.

Eqn.(37) involves various absolute quantities such as the absolute velocity of an object relative to the quantum foam and the absolute speed $c$ also relative to the foam, and of course absolute velocities are excluded from the General Relativity (GR) formalism. However (37) gives (with $t=x_{0}^{0}$ )

$$
d \tau^{2}=d t^{2}-\frac{1}{c^{2}}\left(d \mathbf{r}_{0}(t)-\mathbf{v}\left(\mathbf{r}_{0}(t), t\right) d t\right)^{2}=g_{\mu \nu}\left(x_{0}\right) d x_{0}^{\mu} d x_{0}^{\nu}
$$

which is the Panlevé-Gullstrand form of the metric $g_{\mu \nu}[56,57]$ for GR. All of the above is very suggestive that useful information for the flow dynamics may be obtained from GR by restricting the choice of metric to the Panlevé-Gullstrand form. We emphasize that the absolute velocity $\mathbf{v}_{R}$ has been measured, and so the foundations of GR as usually stated are invalid. As we shall now see the GR formalism involves two phenomena, namely (i) the use of an unnecessarily restrictive Einstein measurement protocol and (ii) the Lorentzian quantum-foam dynamical effects. Later we shall remove this measurement protocol from GR and discover that the GR formalism reduces to explicit fluid flow equations. One significant implication of this is that the whole spacetime formalism introduced by Einstein evaporates - it is nothing more than an epicycle effect, that is, like Ptolemy's epicycles, it is an artifact and arises from not being aware of the influence of certain features of a measurement procedure.

However to understand the GR formalism we need to explicitly introduce the troublesome Einstein measurement protocol and the peculiar effects that it induces in the 
observer's historical records, namely that they have a curved spacetime form. Again we emphasize that experimentally this measurement protocol is unnecessarily restrictive one can do measurements of absolute motion, and then the curvature disappears.

\subsection{The Einstein Measurement Protocol}

The quantum foam, it is argued, induces actual dynamical time dilations and length contractions in agreement with the Lorentz interpretation of special relativistic effects. Then observers in uniform motion 'through' the foam will on measurement of the speed of light obtain always the same numerical value $c$. To see this explicitly consider how various observers $P, P^{\prime}, \ldots$ moving with different speeds through the foam, measure the speed of light. They each acquire a standard rod and an accompanying standardised clock. That means that these standard rods would agree if they were brought together, and at rest with respect to the quantum foam they would all have length $\Delta l_{0}$, and similarly for the clocks. Observer $P$ and accompanying rod are both moving at speed $v_{R}$ relative to the quantum foam, with the rod longitudinal to that motion. $\mathrm{P}$ then measures the time $\Delta t_{R}$, with the clock at end $A$ of the rod, for a light pulse to travel from end $A$ to the other end $B$ and back again to $A$. The light travels at speed $c$ relative to the quantum-foam. Let the time taken for the light pulse to travel from $A \rightarrow B$ be $t_{A B}$ and from $B \rightarrow A$ be $t_{B A}$, as measured by a clock at rest with respect to the quantum foam ${ }^{3}$. The length of the rod moving at speed $v_{R}$ is contracted to

$$
\Delta l_{R}=\Delta l_{0} \sqrt{1-\frac{v_{R}^{2}}{c^{2}}} .
$$

In moving from $A$ to $B$ the light must travel an extra distance because the end $B$ travels a distance $v_{R} t_{A B}$ in this time, thus the total distance that must be traversed is

$$
c t_{A B}=\Delta l_{R}+v_{R} t_{A B}
$$

Similarly on returning from $B$ to $A$ the light must travel the distance

$$
c t_{B A}=\Delta l_{R}-v_{R} t_{B A}
$$

Hence the total travel time $\Delta t_{0}$ is

$$
\begin{aligned}
\Delta t_{0}=t_{A B}+t_{B A} & =\frac{\Delta l_{R}}{c-v_{R}}+\frac{\Delta l_{R}}{c+v_{R}} \\
& =\frac{2 \Delta l_{0}}{c \sqrt{1-\frac{v_{R}^{2}}{c^{2}}}} .
\end{aligned}
$$

\footnotetext{
${ }^{3}$ Not all clocks will behave in this same 'ideal' manner.
} 
Because of the time dilation effect for the moving clock

$$
\Delta t_{R}=\Delta t_{0} \sqrt{1-\frac{v_{R}^{2}}{c^{2}}} .
$$

Then for the moving observer the speed of light is defined as the distance the observer believes the light travelled $\left(2 \Delta l_{0}\right)$ divided by the travel time according to the accompanying clock $\left(\Delta t_{R}\right)$, namely $2 \Delta l_{0} / \Delta t_{R}=c$. So the speed $v_{R}$ of the observer through the quantum foam is not revealed by this procedure, and the observer is erroneously led to the conclusion that the speed of light is always c. This follows from two or more observers in manifest relative motion all obtaining the same speed c by this procedure. Despite this failure this special effect is actually the basis of the spacetime Einstein measurement protocol. That this protocol is blind to the absolute motion has led to enormous confusion within physics. Later we shall see how to overcome the 'blindness' of this procedure, and so manifestly reveal an observer's $v_{R}$.

To be explicit the Einstein measurement protocol actually inadvertently uses this special effect by using the radar method for assigning historical spacetime coordinates to an event: the observer records the time of emission and reception of radar pulses $\left(t_{r}>t_{e}\right)$ travelling through the space of quantum foam, and then retrospectively assigns the time and distance of a distant event $B$ according to (ignoring directional information for simplicity)

$$
T_{B}=\frac{1}{2}\left(t_{r}+t_{e}\right), \quad D_{B}=\frac{c}{2}\left(t_{r}-t_{e}\right),
$$

where each observer is now using the same numerical value of $c$. The event $B$ is then plotted as a point in an individual geometrical construct by each observer, known as a spacetime record, with coordinates $\left(D_{B}, T_{B}\right)$. This is no different to an historian recording events according to some agreed protocol. Unlike historians, who don't confuse history books with reality, physicists do so. We now show that because of this protocol and the quantum foam dynamical effects, observers will discover on comparing their historical records of the same events that the expression

$$
\tau_{A B}^{2}=T_{A B}^{2}-\frac{1}{c^{2}} D_{A B}^{2},
$$

is an invariant, where $T_{A B}=T_{A}-T_{B}$ and $D_{A B}=D_{A}-D_{B}$ are the differences in times and distances assigned to events $A$ and $B$ using the Einstein measurement protocol (52), so long as both are sufficiently small compared with the scale of inhomogeneities in the velocity field.

To confirm the invariant nature of the construct in (53) one must pay careful attention to observational times as distinct from protocol times and distances, and this must be done separately for each observer. This can be tedious. We now demonstrate this for the situation illustrated in Fig.8. 


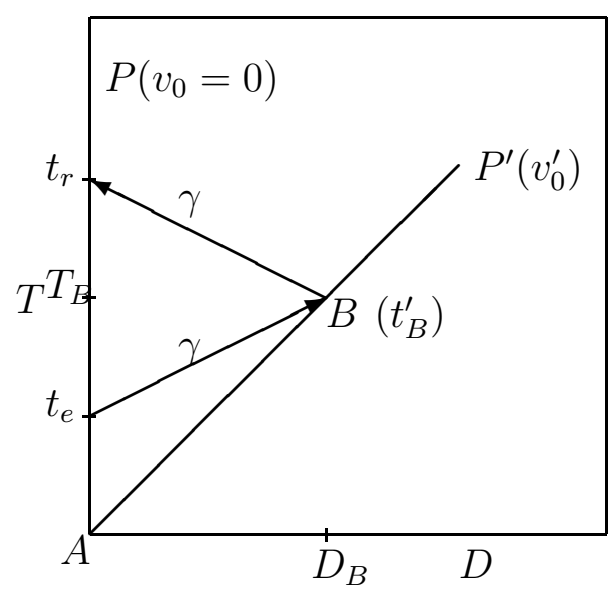

Figure 8: Here $T-D$ is the spacetime construct (from the Einstein measurement protocol) of a special observer $P$ at rest wrt the quantum foam, so that $v_{0}=0$. Observer $P^{\prime}$ is moving with speed $v_{0}^{\prime}$ as determined by observer $P$, and therefore with speed $v_{R}^{\prime}=v_{0}^{\prime}$ wrt the quantum foam. Two light pulses are shown, each travelling at speed $c$ wrt both $P$ and the quantum foam. As we see later these one-way speeds for light, relative to the quantum foam, are equal by observation. Event $A$ is when the observers pass, and is also used to define zero time for each for convenience.

By definition the speed of $P^{\prime}$ according to $P$ is $v_{0}^{\prime}=D_{B} / T_{B}$ and so $v_{R}^{\prime}=v_{0}^{\prime}$, where $T_{B}$ and $D_{B}$ are the protocol time and distance for event $B$ for observer $P$ according to (52). Then using (53) $P$ would find that $\left(\tau_{A B}^{P}\right)^{2}=T_{B}^{2}-\frac{1}{c^{2}} D_{B}^{2}$ since both $T_{A}=0$ and $D_{A}=0$, and whence $\left(\tau_{A B}^{P}\right)^{2}=\left(1-\frac{v_{R}^{\prime 2}}{c^{2}}\right) T_{B}^{2}=\left(t_{B}^{\prime}\right)^{2}$ where the last equality follows from the time dilation effect on the $P^{\prime}$ clock, since $t_{B}^{\prime}$ is the time of event $B$ according to that clock. Then $T_{B}$ is also the time that $P^{\prime}$ would compute for event $B$ when correcting for the time-dilation effect, as the speed $v_{R}^{\prime}$ of $P^{\prime}$ through the quantum foam is observable by $P^{\prime}$. Then $T_{B}$ is the 'common time' for event $B$ assigned by both observers ${ }^{4}$. For $P^{\prime}$ we obtain directly, also from (52) and (53), that $\left(\tau_{A B}^{P^{\prime}}\right)^{2}=\left(T_{B}^{\prime}\right)^{2}-\frac{1}{c^{2}}\left(D_{B}^{\prime}\right)^{2}=\left(t_{B}^{\prime}\right)^{2}$, as $D_{B}^{\prime}=0$ and $T_{B}^{\prime}=t_{B}^{\prime}$. Whence for this situation

$$
\left(\tau_{A B}^{P}\right)^{2}=\left(\tau_{A B}^{P^{\prime}}\right)^{2}
$$

and so the construction (53) is an invariant.

While so far we have only established the invariance of the construct (53) when one of the observers is at rest wrt to the quantum foam, it follows that for two observers

\footnotetext{
${ }^{4}$ Because of gravitational in-flow effects this 'common time' is not the same as a 'universal' or 'absolute time'; see later.
} 
$P^{\prime}$ and $P^{\prime \prime}$ both in motion wrt the quantum foam it follows that they also agree on the invariance of (53). This is easily seen by using the intermediate step of a stationary observer $P$ :

$$
\left(\tau_{A B}^{P^{\prime}}\right)^{2}=\left(\tau_{A B}^{P}\right)^{2}=\left(\tau_{A B}^{P^{\prime \prime}}\right)^{2} .
$$

Hence the protocol and Lorentzian effects result in the construction in (53) being indeed an invariant in general. This is a remarkable and subtle result. For Einstein this invariance was a fundamental assumption, but here it is a derived result, but one which is nevertheless deeply misleading. Explicitly indicating small quantities by $\Delta$ prefixes, and on comparing records retrospectively, an ensemble of nearby observers agree on the invariant

$$
\Delta \tau^{2}=\Delta T^{2}-\frac{1}{c^{2}} \Delta D^{2}
$$

for any two nearby events. This implies that their individual patches of spacetime records may be mapped one into the other merely by a change of coordinates, and that collectively the spacetime patches of all may be represented by one pseudo-Riemannian manifold, where the choice of coordinates for this manifold is arbitrary, and we finally arrive at the invariant

$$
\Delta \tau^{2}=g_{\mu \nu}(x) \Delta x^{\mu} \Delta x^{\nu}
$$

with $x^{\mu}=\left\{T, D_{1}, D_{2}, D_{3}\right\}$.

\subsection{The Origins of General Relativity}

Above it was seen that the Lorentz symmetry of the spacetime construct would arise if the quantum foam system that forms space affects the rods and clocks used by observers in the manner indicated. The effects of absolute motion with respect to this quantum foam are in fact easily observed, and so the velocity $\mathbf{v}_{R}$ of each observer is measurable. However if we work only with the spacetime construct then the effects of the absolute motion are hidden. Einstein was very much misled by the reporting of the experiment by Michelson and Morley of 1887, as now (see later) it is apparent that this experiment, and others since then, revealed evidence of absolute motion. The influence of the Michelson-Morley experiment had a major effect on the subsequent development of physics. One such development was the work of Hilbert and Einstein in finding an apparent generalisation of Newtonian gravity to take into account the apparent absence of absolute motion. Despite the deep error in this work the final formulation, known as General Relativity, has had a number of successes including the perihelion precession of mercury, the bending of light and gravitational red shift. Hence despite the incorrect treatment of absolute motion the formalism of general relativity apparently has some validity. In the next section we shall deconstruct this formalism to discover its underlying physics, but here we first briefly outline the GR formalism. 
The spacetime construct is a static geometrical non-processing historical record, and is nothing more than a very refined history book, with the shape of the manifold encoded in a metric tensor $g_{\mu \nu}(x)$. However in a formal treatment by Einstein the SR formalism and later the GR formalism is seen to arise from three fundamental assumptions:

(1) The laws of physics have the same form in all inertial reference frames.

(2) Light propagates through empty space with a definite speed c independent of the speed of the source or observer.

(3) In the limit of low speeds the new formalism should agree with Newtonian gravity.

We shall see in later sections there is strong evidence that all three of these assumptions are in fact wrong. Nevertheless there is something that is partially correct within the formalism, and that part needs to be extracted and saved, with the rest discarded. From the above assumptions Hilbert and Einstein guessed the equation which specifies the metric tensor $g_{\mu \nu}(x)$, namely the geometry of the spacetime construct,

$$
G_{\mu \nu} \equiv R_{\mu \nu}-\frac{1}{2} R g_{\mu \nu}=\frac{8 \pi G}{c^{2}} T_{\mu \nu}
$$

where $G_{\mu \nu}$ is known as the Einstein tensor, $T_{\mu \nu}$ is the energy-momentum tensor, $R_{\mu \nu}=$ $R_{\mu \alpha \nu}^{\alpha}$ and $R=g^{\mu \nu} R_{\mu \nu}$ and $g^{\mu \nu}$ is the matrix inverse of $g_{\mu \nu}$. The curvature tensor is

$$
R_{\mu \sigma \nu}^{\rho}=\Gamma_{\mu \nu, \sigma}^{\rho}-\Gamma_{\mu \sigma, \nu}^{\rho}+\Gamma_{\alpha \sigma}^{\rho} \Gamma_{\mu \nu}^{\alpha}-\Gamma_{\alpha \nu}^{\rho} \Gamma_{\mu \sigma}^{\alpha},
$$

where $\Gamma_{\mu \sigma}^{\alpha}$ is the affine connection

$$
\Gamma_{\mu \sigma}^{\alpha}=\frac{1}{2} g^{\alpha \nu}\left(\frac{\partial g_{\nu \mu}}{\partial x^{\sigma}}+\frac{\partial g_{\nu \sigma}}{\partial x^{\mu}}-\frac{\partial g_{\mu \sigma}}{\partial x^{\nu}}\right) .
$$

In this formalism the trajectories of test objects are determined by

$$
\Gamma_{\mu \nu}^{\lambda} \frac{d x^{\mu}}{d \tau} \frac{d x^{\nu}}{d \tau}+\frac{d^{2} x^{\lambda}}{d \tau^{2}}=0
$$

which is equivalent to minimising the functional

$$
\tau[x]=\int d t \sqrt{g^{\mu \nu} \frac{d x^{\mu}}{d t} \frac{d x^{\nu}}{d t}}
$$

wrt to the path $x[t]$.

For the case of a spherically symmetric mass a solution of (59) for $g_{\mu \nu}$ outside of that mass $M$ is the Schwarzschild metric

$$
d \tau^{2}=\left(1-\frac{2 G M}{c^{2} r}\right) d t^{2}-\frac{1}{c^{2}} r^{2}\left(d \theta^{2}+\sin ^{2}(\theta) d \phi^{2}\right)-\frac{d r^{2}}{c^{2}\left(1-\frac{2 G M}{c^{2} r}\right)} .
$$


This solution is the basis of various experimental checks of General Relativity in which the spherically symmetric mass is either the sun or the earth. The four tests are: the gravitational redshift, the bending of light, the precession of the perihelion of Mercury, and the time delay of radar signals.

However the solution (64) is in fact completely equivalent to the in-flow interpretation of Newtonian gravity. Making the change of variables $t \rightarrow t^{\prime}$ and $\mathbf{r} \rightarrow \mathbf{r}^{\prime}=\mathbf{r}$ with

$$
t^{\prime}=t+\frac{2}{c} \sqrt{\frac{2 G M r}{c^{2}}}-\frac{4 G M}{c^{2}} \tanh ^{-1} \sqrt{\frac{2 G M}{c^{2} r}},
$$

the Schwarzschild solution (64) takes the form

$$
d \tau^{2}=d t^{\prime 2}-\frac{1}{c^{2}}\left(d r^{\prime}+\sqrt{\frac{2 G M}{r^{\prime}}} d t^{\prime}\right)^{2}-\frac{1}{c^{2}} r^{\prime 2}\left(d \theta^{\prime 2}+\sin ^{2}\left(\theta^{\prime}\right) d \phi^{\prime}\right),
$$

which is exactly the Panlevé-Gullstrand form of the metric $g_{\mu \nu}[56,57]$ in $(45)$ with the velocity field given exactly by the Newtonian form in (34). In which case the trajectory equation (62) of test objects in the Schwarzschild metric is equivalent to solving (42). Thus the minimisation (63) is equivalent to that of (37). This choice of coordinates corresponds to a particular frame of reference in which the test object has velocity $\mathbf{v}_{R}=$ $\mathbf{v}-\mathbf{v}_{0}$ relative to the in-flow field $\mathbf{v}$, as seen in (37).

It is conventional wisdom for practitioners in General Relativity to regard the choice of coordinates or frame of reference to be entirely arbitrary and having no physical significance: no observations should be possible that can detect and measure $\mathbf{v}_{R}$. This 'wisdom' is based on two beliefs (i) that all attempts to detect $\mathbf{v}_{R}$, namely the detection of absolute motion, have failed, and that (ii) the existence of absolute motion is incompatible with the many successes of both the Special Theory of Relativity and of the General Theory of Relativity. Both of these beliefs are demonstrably false.

The results in this section suggest, just as for Newtonian gravity, that the Einstein General Relativity is nothing more than the dynamical equations for a velocity flow field $\mathbf{v}(\mathbf{r}, t)$. Hence the non-flat spacetime construct appears to be merely an unnecessary artifact of the Einstein measurement protocol, which in turn was motivated by the misreporting of the results of the Michelson-Morley experiment. The successes of General Relativity should thus be considered as an insight into the fluid flow dynamics of the quantum foam system, rather than any confirmation of the validity of the spacetime formalism. In the next section we shall deconstruct General Relativity to extract a possible form for this dynamics.

\subsection{Deconstruction of General Relativity}

Here we deconstruct the formalism of General Relativity by removing the obscurification produced by the unnecessarily restricted Einstein measurement protocol. To do this 
we adopt the Panlevé-Gullstrand form of the metric $g_{\mu \nu}$ as that corresponding to the observable quantum foam system, namely to an observationally detected special frame of reference. This form for the metric involves a general velocity field $\mathbf{v}(\mathbf{r}, t)$ where for precision we consider the coordinates $\mathbf{r}, t$ as that of observers at rest with respect to the CMB frame. Note that in this frame $\mathbf{v}(\mathbf{r}, t)$ is not necessarily zero, for mass acts as a sink for the flow. We therefore merely substitute the metric

$$
d \tau^{2}=g_{\mu \nu} d x^{\mu} d x^{\nu}=d t^{2}-\frac{1}{c^{2}}(d \mathbf{r}(t)-\mathbf{v}(\mathbf{r}(t), t) d t)^{2},
$$

into (59) using (61) and (60). This metric involves the arbitrary time-dependent velocity field $\mathbf{v}(\mathbf{r}, t)$. This is a very tedious computation and the results below were obtained by using the symbolic mathematics capabilities of Mathematica. The various components of the Einstein tensor are then

$$
\begin{aligned}
G_{00} & =\sum_{i, j=1,2,3} v_{i} \mathcal{G}_{i j} v_{j}-c^{2} \sum_{j=1,2,3} \mathcal{G}_{0 j} v_{j}-c^{2} \sum_{i=1,2,3} v_{i} \mathcal{G}_{i 0}+c^{2} \mathcal{G}_{00} \\
G_{i 0} & =-\sum_{j=1,2,3} \mathcal{G}_{i j} v_{j}+c^{2} \mathcal{G}_{i 0}, \quad i=1,2,3 . \\
G_{i j} & =\mathcal{G}_{i j}, \quad i, j=1,2,3 .
\end{aligned}
$$

where the $\mathcal{G}_{\mu \nu}$ are given ${ }^{5}$ by

$$
\begin{aligned}
\mathcal{G}_{00}= & \frac{1}{2}\left((\operatorname{tr} D)^{2}-\operatorname{tr}\left(D^{2}\right)\right), \\
\mathcal{G}_{i 0}= & \mathcal{G}_{0 i}=-\frac{1}{2}(\nabla \times(\nabla \times \mathbf{v}))_{i}, \quad i=1,2,3 . \\
\mathcal{G}_{i j}= & \frac{d}{d t}\left(D_{i j}-\delta_{i j} \operatorname{tr} D\right)+\left(D_{i j}-\frac{1}{2} \delta_{i j} \operatorname{tr} D\right) \operatorname{tr} D \\
& -\frac{1}{2} \delta_{i j} \operatorname{tr}\left(D^{2}\right)-(D \Omega-\Omega D)_{i j}, \quad i, j=1,2,3 .
\end{aligned}
$$

Here

$$
D_{i j}=\frac{1}{2}\left(\frac{\partial v_{i}}{\partial x_{j}}+\frac{\partial v_{j}}{\partial x_{i}}\right)
$$

is the symmetric part of the rate of strain tensor $\frac{\partial v_{i}}{\partial x_{j}}$, while the antisymmetric part is

$$
\Omega_{i j}=\frac{1}{2}\left(\frac{\partial v_{i}}{\partial x_{j}}-\frac{\partial v_{j}}{\partial x_{i}}\right)
$$

In vacuum, with $T_{\mu \nu}=0$, we find from (59) and (68) that $G_{\mu \nu}=0$ implies that $\mathcal{G}_{\mu \nu}=0$. It is then easy to check that the in-flow velocity field (34) satisfies these equations. This simply expresses the previous observation that this 'Newtonian in-flow' is completely

\footnotetext{
${ }^{5}$ The $\mathcal{G}_{\mu \nu}$ also arise in the tetrad formulation of GR [58].
} 
equivalent to the Schwarzschild metric. We note that the vacuum equations $\mathcal{G}_{\mu \nu}=0$ do not involve the speed of light; it appears only in (68). It is therefore suggested that (68) amounts to the separation of the Einstein measurement protocol, which involves $c$, from the supposed dynamics of gravity within the GR formalism, and which does not involve $c$. However the details of the vacuum dynamics in (69) have not actually been tested. All the key tests of GR are now seen to amount to a test only of $\delta \tau[x] / \delta x^{\mu}=0$, which is the minimisation of (37), when the in-flow field is given by (68), and which is nothing more than Newtonian gravity. Of course Newtonian gravity was itself merely based upon observations within the solar system, and this may have been too special to have revealed key aspects of gravity. Hence, despite popular opinion, the GR formalism is apparently based upon rather poor evidence.

\subsection{Gravity as Inhomogeneous Quantum-Foam Flow}

Despite the limited insight into gravity which GR is now seen to amount to, here we look for possible generalisations of Newtonian gravity and its in-flow interpretation by examining some of the mathematical structures that have arisen in (69). For the case of zero vorticity $\nabla \times \mathbf{v}=0$ we have $\Omega_{i j}=0$ and also that we may write $\mathbf{v}=\nabla u$ where $u(\mathbf{r}, t)$ is a scalar field, and only one equation is required to determine $u$. To that end we consider the trace of $\mathcal{G}_{i j}$. Note that $\operatorname{tr}(D)=\nabla \cdot \mathbf{v}$, and that

$$
\frac{d(\nabla \cdot \mathbf{v})}{d t}=(\mathbf{v} \cdot \nabla)(\nabla \cdot \mathbf{v})+\frac{\partial(\nabla \cdot \mathbf{v})}{\partial t}
$$

Then using the identity

$$
(\mathbf{v} \cdot \nabla)(\nabla \cdot \mathbf{v})=\frac{1}{2} \nabla^{2}\left(\mathbf{v}^{2}\right)-\operatorname{tr}\left(D^{2}\right)-\frac{1}{2}(\nabla \times \mathbf{v})^{2}+\mathbf{v} \cdot \nabla \times(\nabla \times \mathbf{v}),
$$

and imposing

$$
\sum_{i=1,2,3} \mathcal{G}_{i i}=-8 \pi G \rho
$$

we obtain

$$
\frac{\partial}{\partial t}(\nabla \cdot \mathbf{v})+\frac{1}{2} \nabla^{2}\left(\mathbf{v}^{2}\right)+\frac{\delta}{4}\left((\operatorname{tr} D)^{2}-\operatorname{tr}\left(D^{2}\right)\right)=-4 \pi G \rho .
$$

with $\delta=1$. However $\mathrm{GR}$ in vacuum via $\mathcal{G}_{\mu \nu}=0$ also stipulates that $\frac{1}{4}\left((\operatorname{tr} D)^{2}-\operatorname{tr}\left(D^{2}\right)\right)=$ 0 , implying that in GR $\delta=0$. So (75) with $\delta \neq 0$ is not equivalent to GR. This is nevertheless seen to be a possible generalisation of the Newtonian equation (33) with the new term $C(\mathbf{v})=\frac{\delta}{4}\left((\operatorname{tr} D)^{2}-\operatorname{tr}\left(D^{2}\right)\right)$. The value of $\delta$ should be determined from both the underlying theory and also by analysis of experimental data; see Sects.9.9 and 11.5. We also note that because of the $C(\mathbf{r}) G$ does not necessarily have the same value as the value $G_{N}$ determined by say Cavendish type experiments. 
First note that for the case of the solar system, with the mass concentrated in one object, namely the sun, we see that the in-flow field (34) satisfies (75) since then $C(\mathbf{v})=0$. As we shall see later the presence of the $C$ term is also well hidden when we consider the earth's gravitational effects, although there are various known anomalies that indicate that a generalisation of Newtonian gravity is required. Hence (75) in the case of the solar system is indistinguishable from Newtonian gravity, or the Schwarzschild metric within the General Relativity formalism so long as we use (37), in being able to determine trajectories of test objects. Hence (75) is automatically in agreement with most of the so-called checks on Newtonian gravity and later General Relativity. Note that (75) does not involve the speed of light $c$. Nevertheless we have not derived (75)) from the underlying QHFT, and indeed it is not a consequence of GR, as the $\mathcal{G}_{00}$ equation of (69) requires that $C(\mathbf{v})=0$ in vacuum. Eqn.(75) at this stage should be regarded as a conjecture which will permit the exploration of possible quantum-flow physics and also allow comparison with experiment.

However one key aspect of (75) should be noted here, namely that being a non-linear fluid-flow dynamical system we would expect the flow to be turbulent, particularly when the matter is not spherically symmetric or inside even a spherically symmetric distribution of matter, since then the $C(\mathbf{v})$ term is non-zero and it will drive that turbulence. In the following sections we shall see that the experiments that reveal absolute motion also reveal evidence of turbulence.

\subsection{The 'Dark Matter' Effect}

Because of the $C(\mathbf{v})$ term (75) would predict that the Newtonian inverse square law would not be applicable to systems such as spiral galaxies, because of their highly non-spherical distribution of matter. Of course attempts to retain this law, despite its manifest failure, has led to the spurious introduction of the notion of dark matter within spiral galaxies, and also at larger scales. From

$$
\mathbf{g}=\frac{1}{2} \nabla\left(\mathbf{v}^{2}\right)+\frac{\partial \mathbf{v}}{\partial t}
$$

which is (31) for irrotational flow, we see that (75) gives

$$
\nabla \cdot \mathbf{g}=-4 \pi G \rho-C(\mathbf{v})
$$

and taking running time averages to account for turbulence

$$
\nabla .<\mathbf{g}>=-4 \pi G \rho-<C(\mathbf{v})>,
$$

and writing the extra term as $\langle C(\mathbf{v})\rangle=4 \pi G \rho_{D M}$ we see that $\rho_{D M}$ would act as an effective matter density, and it is suggested that it is the consequences of this term which 
have been misinterpreted as 'dark matter'. Here we see that this effect is actually the consequence of quantum foam effects within the new proposed dynamics for gravity, and which becomes apparent particularly in spiral galaxies. Note that (75) is an equation for $\mathbf{v}$, and now involves the direction of $\mathbf{v}$, unlike the special case of Newtonian gravity (33). Because $\nabla \times \mathbf{v}=0$ we can write (75) in the form

$$
\mathbf{v}(\mathbf{r}, t)=\frac{1}{4 \pi} \int^{t} d t^{\prime} \int d^{3} r^{\prime}\left(\mathbf{r}-\mathbf{r}^{\prime}\right) \frac{\frac{1}{2} \nabla^{2}\left(\mathbf{v}^{2}\left(\mathbf{r}^{\prime}, t^{\prime}\right)\right)+4 \pi G \rho\left(\mathbf{r}^{\prime}, t^{\prime}\right)+C\left(\mathbf{v}\left(\mathbf{r}^{\prime}, t^{\prime}\right)\right)}{\left|\mathbf{r}-\mathbf{r}^{\prime}\right|^{3}},
$$

which allows the determination of the time evolution of $\mathbf{v}$.

The new flow dynamics encompassed in (75) thus accounts for most of the known gravitational phenomena, but will lead to some very clear cut experiments that will distinguish it from the two previous attempts to model gravitation. It turns out that these two attempts were based on some key 'accidents' of history. In the case of the Newtonian modelling of gravity the prime 'accident' was of course the solar system with its high degree of spherical symmetry. In each case we had test objects, namely the planets, in orbit about the sun, or we had test object in orbit about the earth. In the case of the General Relativity modelling the prime 'accident' was the mis-reporting of the Michelson-Morley experiment, and the ongoing belief that the so called 'relativistic effects' are incompatible with absolute motion. We shall consider in detail later some further anomalies that might be appropriately explained by this new modelling of gravity. Of course that the in-flow has been present in various experimental data is also a significant argument for something like (75) to model gravity. Key new experimental techniques will be introduced later which will enable the consequences of (75) to be tested. If necessary these experiments will provide insights into possible modifications to (75).

\subsection{In-Flow Superposition Approximation}

We consider here why the existence of absolute motion and as well the presence of the $C(\mathbf{v})$ term appears to have escaped attention in the case of gravitational experiments and observations near the earth, despite the fact, in the case of the $C(\mathbf{v})$ term, that the presence of the earth breaks the spherical symmetry of the matter distribution of the sun.

First note that if we have a matter distribution $\rho(\mathbf{r})$ at rest in the space of quantum foam, and that (75) has solution $\mathbf{v}_{0}(\mathbf{r}, t)$, and then with $\mathbf{g}_{0}(\mathbf{r}, t)$ given by $(76)$, then when the same matter distribution is uniformly translating at velocity $\mathbf{V}$, that is $\rho(\mathbf{r}) \rightarrow$ $\rho(\mathbf{r}-\mathbf{V} t)$, then a solution to $(75)$ is

$$
\mathbf{v}(\mathbf{r}, t)=\mathbf{v}_{0}(\mathbf{r}-\mathbf{V} t, t)+\mathbf{V}
$$

Note that this is a manifestly time-dependent process and the time derivative in (31) or (42) and (75) plays an essential role. As well the result is nontrivial as (75) is a non-linear 
equation. The solution (80) follows because (i) the expression for the acceleration $\mathbf{g}(\mathbf{r}, t)$ gives, and this expression occurs in (75),

$$
\begin{aligned}
\mathbf{g}(\mathbf{r}, t) & =\frac{\partial \mathbf{v}_{0}(\mathbf{r}-\mathbf{V} t, t)}{\partial t}+\left(\left(\mathbf{v}_{0}(\mathbf{r}-\mathbf{V} t, t)+\mathbf{V}\right) . \nabla\right)\left(\mathbf{v}_{0}(\mathbf{r}-\mathbf{V} t, t)+\mathbf{V}\right) \\
& =\left.\frac{\partial \mathbf{v}_{0}\left(\mathbf{r}-\mathbf{V} t^{\prime}, t\right)}{\partial t^{\prime}}\right|_{t^{\prime} \rightarrow t}+\mathbf{g}_{0}(\mathbf{r}-\mathbf{V} t, t)+(\mathbf{V} \cdot \nabla) \mathbf{v}_{0}(\mathbf{r}-\mathbf{V} t, t) \\
& =-(\mathbf{V} \cdot \nabla) \mathbf{v}_{0}(\mathbf{r}-\mathbf{V} t, t)+\mathbf{g}_{0}(\mathbf{r}-\mathbf{V} t, t)+(\mathbf{V} \cdot \nabla) \mathbf{v}_{0}(\mathbf{r}-\mathbf{V} t, t) \\
& =\mathbf{g}_{0}(\mathbf{r}-\mathbf{V} t, t)
\end{aligned}
$$

as there is a key cancellation of two terms in (81), and (ii) clearly $C\left(\mathbf{v}_{0}(\mathbf{r}-\mathbf{V} t, t)+\mathbf{V}\right)=$ $C\left(\mathbf{v}_{0}(\mathbf{r}-\mathbf{V} t, t)\right)$, and so this term is also simply translated. Hence apart from the translation effect the acceleration is the same. Hence the velocity vector addition rule in (80) is valid for generating the vector flow field for the translating matter distribution. This is why the large absolute motion velocities of some $400 \mathrm{~km} / \mathrm{s}$ do not interfer with the usual computation and observation of gravitational forces.

For earth based gravitational phenomena the motion of the earth takes place within the velocity in-flow towards the sun, and the velocity sum rule (80) is only approximately valid as now $\mathbf{V} \rightarrow \mathbf{V}(\mathbf{r}, t)$ and no longer corresponds to uniform translation, and manifests turbulence. To be a valid approximation the inhomogeneity of $\mathbf{V}(\mathbf{r}, t)$ must be much smaller than that of $\mathbf{v}_{0}(\mathbf{r}-\mathbf{V} t, t)$, which it is, as the earth's centripetal acceleration about the sun is approximately 1/1000 that of the earth's gravitational acceleration at the surface of the earth. Nevertheless turbulence associated with the $C(\mathbf{v})$ term is apparent in experimental data. The validity of this approximation demonstrates that the detection of a cosmic absolute motion and the in-flow theory of gravity are consistent with the older methods of computing gravitational forces. This is why both the presence of the $C(\mathbf{v})$ term, the in-flow and the absolute motion have gone almost unnoticed in earth based gravitational experiments, except for various anomalies; see section 9.9.

\subsection{Gravitational In-Flow and the GPS}

It has been extensively argued that the very successful operation of the Global Positioning System (GPS) [59] is proof of the validity of the General Relativity formalism for gravity. However as is well known, and was most clearly stated by Popper, in science agreement with observation does not amount to the proof of the theory used to successfully describe the experimental data; in fact experiment can only strictly be used to disprove a theory.

We show here that the new in-flow theory of gravity together with the observed absolute velocity of motion of the solar system through space are together compatible with the operation of the Global Positioning System (GPS) [17]. Given the developments 
above this turns out to be an almost trivial exercise. As usual in this system the effects of the sun and moon are neglected. Various effects need to be included as the system relies upon extremely accurate atomic clocks in the satellites forming the GPS constellation. Within both the new theory and General Relativity these clocks are effected by both their speed and the gravitational effects of the earth. As well the orbits of these satellites and the critical time delays of radio signals from the satellites need to be computed. For the moment we assume spherical symmetry for the earth. The effects of non-sphericity will be discussed below. In General Relativity the orbits and signalling time delays are determined by the use of the geodesic equation (62) and the Schwarzschild metric (64). However these two equations are equivalent to the orbital equation (44) and the velocity field (80), with a velocity $\mathbf{V}$ of absolute motion, and with the in-flow given by (34), noting the result in section 9.7. For EM signalling the elapsed time in (37) requires careful treatment. Hence the two systems are completely mathematically equivalent: the computations within the new system may most easily be considered by relating them to the mathematically equivalent General Relativity formalism. We can also see this by explicitly changing from the CMB frame to a non-rotating frame co-moving with the earth by means of the change of variables

$$
\begin{aligned}
\mathbf{r} & =\mathbf{r}^{\prime}+\mathbf{V} t^{\prime} \\
t & =t^{\prime} \\
\mathbf{v} & =\mathbf{v}^{\prime}+\mathbf{V}
\end{aligned}
$$

which lead to the relationships of differentials

$$
\begin{aligned}
\nabla^{\prime} & =\nabla \\
\frac{\partial}{\partial t^{\prime}} & =\frac{\partial}{\partial t}+\mathbf{V} \cdot \nabla
\end{aligned}
$$

These expressions then lead to the demonstration of the invariance of (75). Then in the earth co-moving frame the absolute velocity $\mathbf{V}$ does not appear in (75). Then another change of variables, as in (65), permits (75) to be written in the form of General Relativity with a Schwarzschild metric.

The consistency between the absolute motion velocity $\mathbf{V}$ and General Relativity may also be directly checked by showing explictly, using say Mathematica, that the metric

$$
d \tau^{2}=g_{\mu \nu} d x^{\mu} d x^{\nu}=d t^{2}-\frac{1}{c^{2}}\left(d \mathbf{r}(t)-((\mathbf{v}(\mathbf{r}-\mathbf{V} t)+\mathbf{V}) d t)^{2},\right.
$$

is a solution to (59) for $T_{\mu \nu}=0$, ie outside matter, where $\mathbf{v}(\mathbf{r})$ is the in-flow velocity field in (34). This metric is a generalisation of the Panlevé-Gullstrand metric to include the absolute motion effect. This emphasises yet again that for a spherically symmetric matter 
distribution the Schwarzschild metric, which is equivalent to the Panlevé-Gullstrand metric, is physically identical to Newtonian gravity.

There are nevertheless two differences between the two theories. One is their different treatment of the non-sphericity of the earth via the $C(\mathbf{v})$ term, and the second difference is the effects of the in-flow turbulence. In the operation of the GPS the density $\rho(\mathbf{r})$ of the earth is not used. Rather the gravitational potential $\Phi(\mathbf{r})$ is determined observationally. In the new gravity theory the determination of such a gravitational potential via (75) and $\Phi(\mathbf{r})=-\frac{1}{2} \mathbf{v}^{2}(\mathbf{r})$ would involve the extra $C(\mathbf{v})$ term. Hence because of this phenomenological treatment the effects of the $C(\mathbf{v})$ term are not checkable. However the gravitational wave effect is expected to affect the operation of the GPS, and the GPS constellation would offer a worldwide network which would enable the investigation of the spatial and temporal correlations of these gravitational waves.

There is also a significant interpretational difference between the two theories. For example in General Relativity the relativistic effects involve both the 'special relativity' orbital speed effect via time dilations of the satellite clocks together with the General Relativity 'gravitational potential energy' effect on the satellite clocks. In the new theory there is only one effect, namely the time dilation effect produced by the motion of the clocks through the quantum foam, and the speeds of these clocks involve the vector sum of the orbital velocity and the velocity caused by the in-flow of the quantum foam into the earth. This is illustrated by figure 14, where now the orbit refers to that of a satellite about the earth.

\subsection{Measurements of $G$}

As noted in section 9.1 Newton's Inverse Square Law of Gravitation may only be strictly valid in cases of spherical symmetry. The theory that gravitational effects arise from inhomogeneities in the quantum foam flow implies that there is no 'universal law of gravitation' because the inhomogeneities are determined by non-linear 'fluid equations' and the solutions have no form which could be described by a 'universal law'. Fundamentally there is no generic fluid flow behaviour. The Inverse Square Law is then only an approximation, with large deviations expected in the case of spiral galaxies. Nevertheless Newton's gravitational constant $G$ will have a definite value as it quantifies the effective rate at which matter dissipates the information content of space.

From these considerations it follows that the measurement of the value of $G$ will be difficult as the measurement of the forces between two of more objects, which is the usual method of measuring $G$, will depend on the geometry of the spatial positioning of these objects in a way not previously accounted for because the Newtonian Inverse Square Law has always been assumed, or in some cases a specified change in the form of the law has 


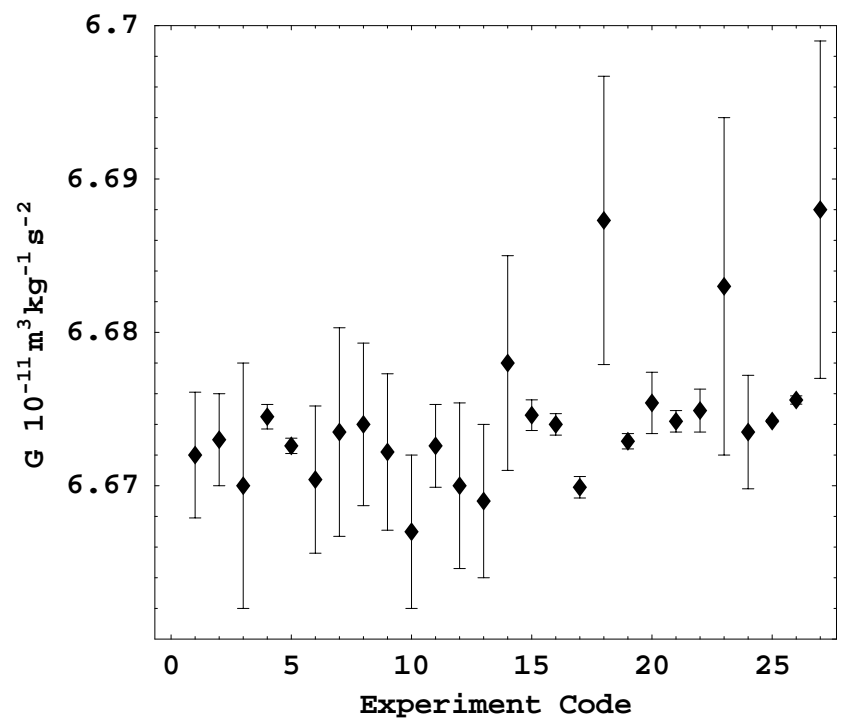

Figure 9: Results of precision measurements of $G$ published in the last sixty years in which the Newtonian theory was used to analyse the data. These results show the presence of a systematic effect not in the Newtonian theory. 1: Gaithersburg 1942 [60], 2: Magny-les-Hameaux 1971 [61], 3: Budapest 1974 [62], 4: Moscow 1979 [63], 5: Gaithersburg 1982 [64], 6-9: Fribourg Oct 84, Nov 84, Dec 84, Feb 85 [65], 10: Braunschweig 1987 [66], 11: Dye 3 Greenland 1995 [67], 12: Gigerwald Lake 1994 [68], 13-14: Gigerwald lake19 95 112m, 88m [69], 15: Lower Hutt 1995 MSL [70], 16: Los Alamos 1997 [71], 17: Wuhan 1998 [72], 18: Boulder JILA 1998 [73], 19: Moscow 1998 [74], 20: Zurich 1998 [75], 21: Lower Hutt MSL 1999 [76], 22: Zurich 1999 [77], 23: Sevres 1999 [78], 24: Wuppertal 1999 [79], 25: Seattle 2000 [80], 26: Sevres 2001 [81], 27: Lake Brasimone 2001 [82]. Data compilation adapted from [83].

been used. But in all cases a 'law' has been assumed, and this may have been the flaw in the analysis of data from such experiments. This implies that the value of $G$ from such experiments will show some variability as a systematic effect has been neglected in analysing the experimental data, for in none of these experiments is spherical symmetry present. So experimental measurements of $G$ should show an unexpected contextuality. As well the influence of surrounding matter has also not been properly accounted for. Of course any effects of turbulence in the inhomogeneities of the flow has presumably also never even been contemplated. The first measurement of $G$ was in 1798 by Cavendish using a torsional balance. As the precision of experiments increased over the years and a variety of techniques used the disparity between the values of $G$ has actually increased [84]. Figure 9 shows the results from precision measurements of $G$ over the last 60 years. As can be seen one indication of the contextuality is that measurements of $G$ produce 
values that differ by nearly 40 times their individual error estimates. In 1998 CODATA increased the uncertainty in $G$ from $0.013 \%$ to $0.15 \%$. It is predicted that these $G$ anomalies will only be resolved when the new theory of gravity is used in analysing the data from these experiments.

In Sect.11.5 we show that the borehole/mine $g$ anomaly is capable of determining both the value of $G$ and the value of the new parameter $\delta$.

\section{Detection of Absolute Motion}

\subsection{Space and Absolute Motion}

The phenomenon of space has resisted understanding for thousands of years. Properties of space were observed and used by the Babylonians and Egyptians, apparently in the latter case to enable the agricultural boundaries to be re-established after flooding of the Nile Valley. Eventually these properties of space were encoded and formalised in the mathematics of geometry with its abstract notions of a geometrical space, points, lines, etc. A major compilation of the results was written by Euclid in 300 BCE. This was the first major modelling of physical phenomena by a mathematical system, and gave us the mindset of syntactical formalism that prevails in physics to this day. Eventually the mathematical model became identified with the phenomenon itself, that is, physical space was understood to be a geometrical space. And so the distinction between physical space and geometrical space disappeared. This confusion of a phenomenon with its mathematical modelling is a re-occurring problem in physics. Given that now physical space was considered to be a Euclidean geometrical system, and as such devoid of structure, thinkers like Descartes and Newton appreciated that such a modelling appeared to be deficient. To improve the modelling they embellished the old Aristotelian notion that space was occupied by an aether. This dualism with both space and aether became a major concept in physics. It does not appear to have occurred to the early investigators that something other than geometrical space could underpin the phenomenon of physical space. In the same era Galileo introduced the modelling of time by the geometrical onedimensional space, and Newton made extensive use of this modelling in his development of dynamics. Like space the phenomenon of time eventually became synonymous with its geometrical model until today most physicists regard time as a geometrical phenomenon, which of course necessitates the denial of any aspects of time that the geometrical model cannot accommodate. Of course that is how non-process physics was arrived at.

Descartes considered the aether to have the structure of whirling large and small vortices, and that the motion of these vortices for instance carried the planets in their orbits about the sun, as illustrated in Fig.10. 


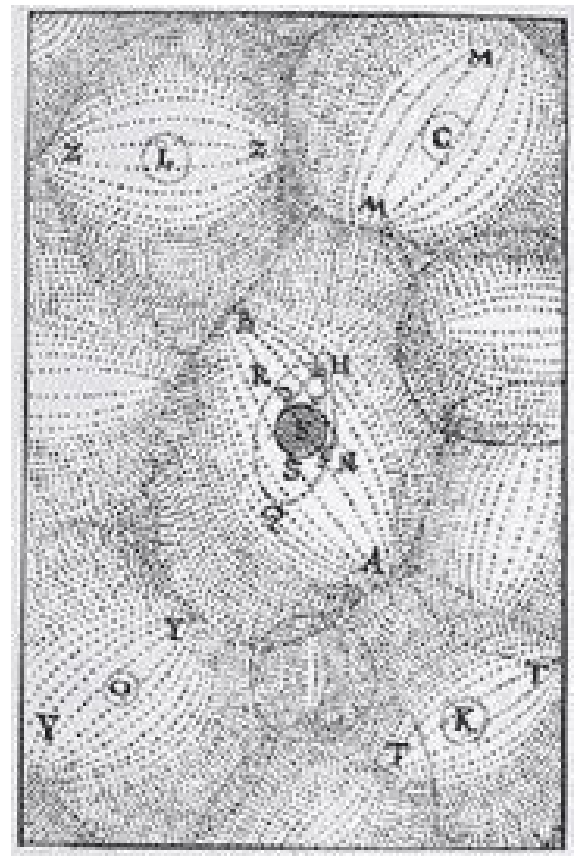

Figure 10: Descartes' vortices carry the planets about the sun. Plate VI from [85].

Newton in a letter in 1675 to Oldenburg, Secretary of the Royal Society, and later to Robert Boyl, used the aether to offer a different explanation of planetary motion, namely that the gravitational theory he had developed was to be explained as being the result of a

condensation causing a flow of an ether with a corresponding thinning of the ether density associated with the increased velocity of flow

though Newton was of the opinion that this aether flow was undetectable.

The concept of the aether was generally accepted, and following Maxwell's successful development of the unifying theory for electromagnetic phenomena which predicted a unique speed $c$ for light, the need for the aether became even greater as it was believed that this speed referred to the motion of light relative to the aether. It was the argued by Maxwell that the motion of the earth through the aether should be measurable by means of the change in speed of the light as measured by an observer travelling with the earth. This is the concept of absolute motion, namely that motion with respect to space itself is meaningful and measurable. The size of this effect is $v / c=1 / 10,000$, where $v=30 \mathrm{~km} / \mathrm{s}$ is the orbital speed of the earth, and $c=300,000 \mathrm{~km} / \mathrm{s}$. Maxwell appreciated that an experiment that utilised a ray of light going outward and then returning would result in the effect becoming only second order in $v / c$, and since $(v / c)^{2}=10^{-8}$ it was thought to be too small for measurement. 
Michelson responded to Maxwell's challenge and in 1880-1881 he devised the instrument now known as the Michelson interferometer to detect this absolute motion [87]. The dualism of space and aether was still favoured, so such experiments became known as aether drift experiments. As explained in detail later a beam of light is split into two, sent in orthogonal directions, and reflected back to a common place. Differences in travel times for the two beams were expected to result in interference fringes being formed, as was seen. However the key operational effect is that the fringes should drift across the field of view as the interferometer is rotated, if absolute motion existed as a phenomenon. The magnitude of the maximum fringe shift is then a measure of the absolute speed of the earth. An interferometer with arms of length $L=1.2 \mathrm{~m}$ was built and operated in Germany by Michelson, but the effects of vibrations made the results inconclusive. In 1887 Michelson and Morley having constructed a new interferometer with arms with an effective length of $L=11 \mathrm{~m}$, achieved by the use of multiple reflections from mirrors, performed their key experiment in the Main Building of Adelbert College in Cleveland. No other experiment in the history of physics has ever acquired the significance and consequences of this one experiment.

The experiment involved observations over brief periods over four days in July 1887, and involved in all only 36 turns of the interferometer. Remarkably the results [88] showed the shift of the interference fringes characteristic of absolute motion, and analysis of the results using Newtonian physics gave a speed of approximately $8 \mathrm{~km} / \mathrm{s}$, which is certainly a very high speed, but significantly less than the orbital speed of the earth. Absolute motion had been discovered, but clearly there was a problem with the magnitude of the effect according to the prevailing theory of the interferometer. How could the interferometer determined speed be less than the orbital speed? It could have just been the bad luck of the month of July when perhaps the solar system and the earth had a net vectorially summed speed of $8 \mathrm{~km} / \mathrm{s}$. Michelson could easily have resolved this possibility be repeating the experiment over a year, during which time the vectorial sum of the two velocities would have changed and the vector sum effect would have been observable. It would be over 100 years before the smallness of this interferometer determined speed was finally understood. Indeed the Michelson-Morley data corresponds to an actual absolute speed of some $400 \mathrm{~km} / \mathrm{s}$. The analysis by Michelson of the experimental data thus turned out to be totally flawed. Michelson had placed total faith in Newtonian physics, and in particular in Galilean Relativity, and never countenanced the possibility that it could require revision, that his experiment was in fact revealing not the absence of absolute motion but the very failure of Newtonian physics. The failure by Michelson to understand this point had disastrous consequences for the development of physics throughout the twentieth century. Physics has yet to recover from the misreporting and misinterpretation of this experiment. 
Dayton Miller knew that the Michelson-Morley experiment had in fact detected absolute motion and set out to build even larger Michelson interferometers. Miller also understood that the physical principles involved in the interferometer were not understood, and he introduced the $k$ parameter to allow for this. In one of the greatest experiments ever in physics Miller successfully operated his interferometer over many years taking detailed data from thousands of rotations at all times of the day and throughout the year. As Miller argued an understanding of the value of the $k$ parameter would have to wait further developments in physics, but in the meantime he could determine the magnitude and direction of the absolute speed of the solar system by using the modulating effect of the earth's yearly rotation about the sun. The same analysis also permitted the effective value of $k$ to be determined. Miller's analysis, published [89] in 1933, resulted in an absolute speed of some $200 \mathrm{~km} / \mathrm{s}$ in a direction near to the South Celestial Pole.

Miller's discovery was ignored and after his death his experiments were attacked for being poorly executed. Why was this? And why were the many successful repetitions of the detection of absolute motion ignored? Michelson himself was devoted to the task of detecting absolute motion and repeatedly built and operated new interferometers until his death in 1941. Each one of these interferometers did in fact reveal the effects of absolute motion, but Michelson's method of analysis had remained flawed as he had ignored Miller's treatment of the problem of an inadequate understanding of actually how an interferometer operated. Michelson died not realising that he had again and again observed absolute motion. Ironically during his life he had been richly rewarded by a Nobel prize for reporting that the effects of absolute motion had not been observed. Miller who had perfected the Michelson interferometer and who had worked around the theoretical limitations on the understanding of its operation and who had correctly analysed the vast amount of his data to reveal the magnitude and direction of motion of the solar system, and who had detected the rotational motion of the earth in its orbit about the sun via a purely laboratory based experiment was completely castigated by the physics community.

The operation of the Michelson interferometer was not finally understood until 2002 when the theoretical explanation for Miller's $k$ parameter was finally discovered [12]. Briefly the interferometer involves three key physical effects (i) a change in the difference of the geometrical path length of the two light beams when the interferometer is in absolute motion and (ii) a Fitzgerald-Lorentz real physical contraction of the arm parallel to the direction of motion. The final third and last effect is critical: (iii) the first two effects cancel if the interferometer is operated in vacuum, but do not cancel if the light passes through air or some other gas, and only then is absolute motion detectable by the interferometer. Both the Michelson-Morley and Miller experiments were done in air. It turns out that Miller's $k$ parameter is determined by the refractive index $n$ of the 
gas according to $k=\sqrt{n\left(n^{2}-1\right)}$. Finally after over 100 years the diverse gas-mode interferometer data could be analysed and understood. One of the most significant discoveries [14] to come from that re-analysis is that as well as detecting the absolute motion of the solar system through space and the orbital speed of the earth in its orbit about the sun the data also reveals an inflow past the earth towards the sun. This is the very in-flow that Newton had contemplated. It is observable and it is the explanation for gravity. As well the interferometer data shows that the direction of absolute motion relative to local space, after taking account of the in-flow towards the sun, is different to the direction of motion relative to the universe as a whole. This is because of the gravitational quantum-foam in-flow into the Milky Way galaxy.

But why had all these discoveries not emerged at the beginning of the twentieth century when all the clues and theoretical insights had begun to emerge? The explanation is clear in the history books. In response to Michelson's bungled analysis and building upon the many theoretical insights by Fitzgerald, Lorentz, Larmor and others the Einsteins usurped and formalised the work of all these physicists by asserting that absolute motion was without meaning and consequently not observable. The formalism introduced the geometrical spacetime construct which amounted to a truly bizarre reinterpretation of the relativistic effects that Fitzgerald [94], Lorentz [95] and Larmor [96] had introduced. Eventually the Einstein interpretation came to be accepted over the more physical interpretation that the effects on clocks and rods were real physical effects caused by absolute motion. Indeed in an even more bizarre turn of events it became accepted within physics, as a direct consequence of the Einstein postulates, that the relativistic effects were incompatible with absolute motion. Within a decade or so of the introduction of the Einstein re-interpretation absolute motion had become a banned concept in physics, and still is to this day. By the time Miller's extraordinary interferometer experiments began to produce such dazzling evidence of absolute motion effects the prevailing belief in physics was that absolute motion was mutually exclusive to the various experimentally confirmed relativistic effects. So Miller had to be proved wrong. In one of the most perverse papers ever published Shankland and others [90] set out to do just that ${ }^{6}$, and after Miller had died. This was despite the fact that Shankland had been a research associate of Miller and had participated in the final determination of the absolute motion of the solar system.

The operation of the Michelson interferometer is analysed in the following sections, and the various results that follow from analysis of the data are presented. As well other experiments that confirm the Miller results are introduced including in particular a recent 1991 first-order experiment by Roland DeWitte. It is now clear that first-order

\footnotetext{
${ }^{6}$ Einstein had been very concerned about the results that Miller was obtaining and in a number of letters to Shankland he expressed his gratitude to Shankland for dealing with them.
} 

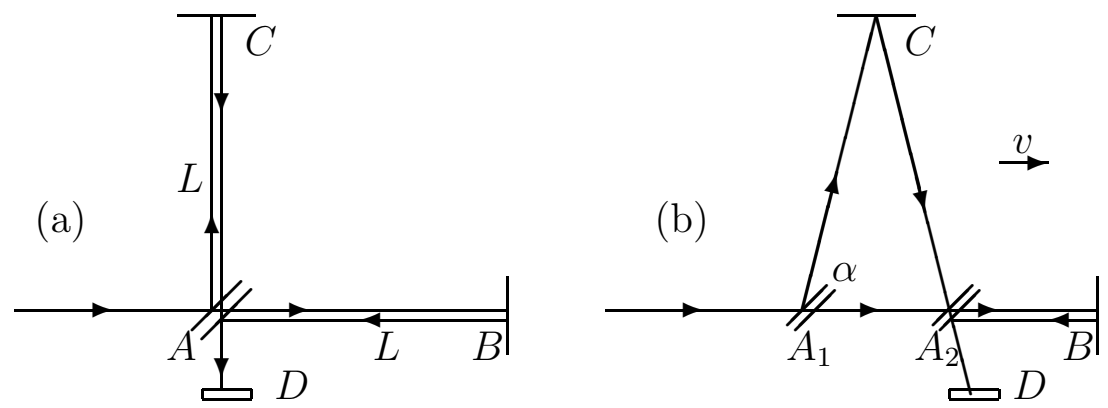

Figure 11: Schematic diagrams of the Michelson Interferometer, with beamsplitter/mirror at $A$ and mirrors at $B$ and $C$ on arms from $A$, with the arms of equal length $L$ when at rest. $D$ is a quantum detector that causes localisation of the photon state by a collapse process. In (a) the interferometer is at rest in space. In (b) the interferometer is moving with speed $v$ relative to space in the direction indicated. Interference fringes are observed at the quantum detector $D$. If the interferometer is rotated in the plane through $90^{\circ}$, the roles of arms $A C$ and $A B$ are interchanged, and during the rotation shifts of the fringes are seen in the case of absolute motion, but only if the apparatus operates in a gas. By counting fringe changes the speed $v$ may be determined.

devices that are far superior to the Michelson interferometer will greatly simplify the experimental study of various physical phenomena that are manifest in the absolute motion effects. In particular the gravitationally related in-flow past the earth towards the sun and the turbulence that appears to be present in that in-flow will be studied. As explained earlier the in-flow effect is essentially related to quantum gravity effects, and so the first-order devices give us for the first time techniques for investigating quantum gravity. Of particular significance is that the direction of the absolute motion of the solar system as determined by the Miller interferometer studies is in a direction different from that of the motion of the solar system with respect to the Cosmic Microwave Background defined frame of reference. This again is a manifestation of gravitational in-flows, this time into the Milky Way galaxy.

\subsection{Theory of the Michelson Interferometer}

We now show for the first time in over 100 years how the three key effects together permit the Michelson interferometer [87] to reveal the phenomenon of absolute motion when operating in the presence of a gas, with the third effect only discovered in 2002 [12]. The main outcome is the derivation of the origin of the Miller $k^{2}$ factor in the expression 
for the time difference for light travelling via the orthogonal arms,

$$
\Delta t=k^{2} \frac{L\left|\mathbf{v}_{P}\right|^{2}}{c^{3}} \cos (2(\theta-\psi)) \text {. }
$$

Here $\mathbf{v}_{P}$ is the projection of the absolute velocity $\mathbf{v}$ of the interferometer through the quantum-foam onto the plane of the interferometer, where the projected velocity vector $\mathbf{v}_{P}$ has azimuth angle $\psi$ relative to the local meridian, and $\theta$ is the angle of one arm from that meridian. The $k^{2}$ factor is $k^{2}=n\left(n^{2}-1\right)$ where $n$ is the refractive index of the gas through which the light passes, $L$ is the length of each arm and $c$ is the speed of light relative to the quantum foam. This expression follows from three key effects: (i) the difference in geometrical length of the two paths when the interferometer is in absolute motion, as first realised by Michelson, (ii) the Fitzgerald-Lorentz contraction of the arms along the direction of motion, and (iii) that these two effects precisely cancel in vacuum, but leave a residual effect if operated in a gas, because the speed of light through the gas is reduced to $V=c / n$, ignoring here for simplicity any Fresnel-drag effects, Sect.16.2, which are analysed in Sect.16.4. This is one of the aspects of the quantum foam physics that distinguishes it from the Einstein formalism. The time difference $\Delta t$ is revealed by the fringe shifts on rotating the interferometer. In Newtonian physics, that is with no Fitzgerald-Lorentz contraction, $k^{2}=n^{3}$, while in Einsteinian physics $k=0$ reflecting the fundamental assumption that absolute motion is not measurable and indeed has no meaning. The Special Relativity null effect for the interferometer is explicitly derived in Sect.16.5. So the experimentally determined value of $k$ is a key test of fundamental physics. Table 1 summarises the differences between the three fundamental theories in their modelling of time, space, gravity and the quantum, together with their distinctive values for the interferometer parameter $k^{2}$. For air $n=1.00029$, and so for process physics $k=0.0241$ and $k^{2}=0.00058$, which is close to the Einsteinian value of $k=0$, particularly in comparison to the Newtonian value of $k=1.0$. This small but non-zero $k$ value explains why the Michelson interferometer experiments gave such small fringe shifts. Fortunately it is possible to check the $n$ dependence of $k$ as one experiment [91] was done in Helium gas, and this has an $n^{2}-1$ value significantly different from that of air.

\begin{tabular}{|l|c|c|c|c|c|}
\hline Theory & Time & Space & Gravity & Quantum & $k^{2}$ \\
\hline \hline Newton & geometry & geometry & force & Quantum Theory & $n^{3}$ \\
\hline Einstein & \multicolumn{2}{|c|}{ curved geometry } & curvature & Quantum Field Theory & 0 \\
\hline Process & process & $\begin{array}{c}\text { quantum } \\
\text { foam }\end{array}$ & $\begin{array}{c}\text { inhomogeneous } \\
\text { flow }\end{array}$ & $\begin{array}{c}\text { Quantum Homotopic } \\
\text { Field Theory }\end{array}$ & $n\left(n^{2}-1\right)$ \\
\hline
\end{tabular}

Table 1: Comparisons of Newtonian, Einsteinian and Process Physics.

In deriving (86) in the new physics it is essential to note that space is a quantum-foam system which exhibits various subtle features. In particular it exhibits real dynamical 
effects on clocks and rods. In this physics the speed of light is only $c$ relative to the quantum-foam, but to observers moving with respect to this quantum-foam the speed appears to be still $c$, but only because their clocks and rods are affected by the quantumfoam. As shown in Sect.9.2 such observers will find that records of observations of distant events will be described by the Einstein spacetime formalism, but only if they restrict measurements to those achieved by using clocks, rods and light pulses, that is using the Einstein measurement protocol. However if they use an absolute motion detector then such observers can correct for these effects.

It is simplest in the new physics to work in the quantum-foam frame of reference. If there is a gas present at rest in this frame, such as air, then the speed of light in this frame is $V=c / n$. If the interferometer and gas are moving with respect to the quantum foam, as in the case of an interferometer attached to the earth, then the speed of light relative to the quantum-foam is still $V=c / n$ up to corrections due to drag effects. Hence this new physics requires a different method of analysis from that of the Einstein physics. With these cautions we now describe the operation of a Michelson interferometer in this new physics, and show that it makes predictions different to that of the Einstein physics. Of course experimental evidence is the final arbiter in this conflict of theories.

As shown in Fig.12 the beamsplitter/mirror when at $A$ sends a photon $\psi(t)$ into a superposition $\psi(t)=\psi_{1}(t)+\psi_{2}(t)$, with each component travelling in different arms of the interferometer, until they are recombined in the quantum detector which results in a localisation process, and one spot in the detector is produced ${ }^{7}$. Repeating with many photons reveals that the interference between $\psi_{1}$ and $\psi_{2}$ at the detector results in fringes. These fringes actually only appear if the mirrors are not quite orthogonal, otherwise the screen has a uniform intensity and this intensity changes as the interferometer is rotated, as shown in the analysis by Hicks [93]. To simplify the analysis here assume that the two arms are constructed to have the same lengths $L$ when they are physically parallel to each other and perpendicular to $v$, so that the distance $B B^{\prime}$ is $L \sin (\theta)$. The Fitzgerald-Lorentz effect in the new physics is that the distance $S B^{\prime}$ is $\gamma^{-1} L \cos (\theta)$ where $\gamma=1 / \sqrt{1-v^{2} / c^{2}}$. The various other distances are $A B=V t_{A B}, B C=V t_{B C}, A S=v t_{A B}$ and $S C=v t_{B C}$, where $t_{A B}$ and $t_{B C}$ are the travel times. Applying the Pythagoras theorem to triangle $A B B^{\prime}$ we obtain

$$
t_{A B}=\frac{2 v \gamma^{-1} L \cos (\theta)+\sqrt{4 v^{2} \gamma^{-2} L^{2} \cos ^{2}(\theta)+4 L^{2}\left(1-\frac{v^{2}}{c^{2}} \cos ^{2}(\theta)\right)\left(V^{2}-v^{2}\right)}}{2\left(V^{2}-v^{2}\right)} .
$$

The expression for $t_{B C}$ is the same except for a change of sign of the $2 v \gamma^{-1} L \cos (\theta)$ term,

\footnotetext{
${ }^{7} \mathrm{~A}$ simplified analysis when the arms are parallel/orthogonal to the direction of motion is given in Sect.16.3.
} 


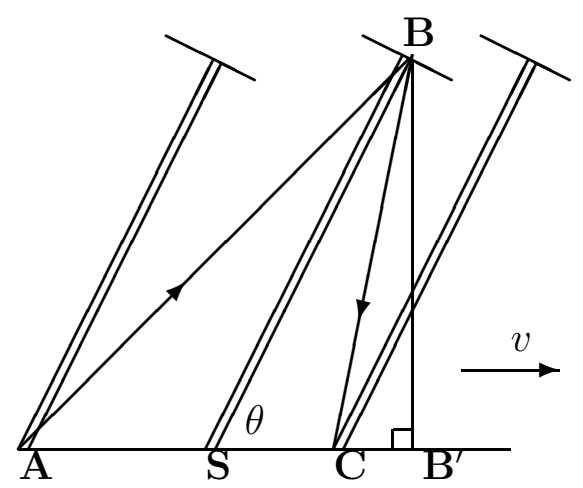

Figure 12: One arm of a Michelson Interferometer travelling at angle $\theta$ and velocity $\mathbf{v}$, and shown at three successive times: (i) when photon leaves beamsplitter at $A$, (ii) when photon is reflected at mirror $B$, and (iii) when photon returns to beamsplitter at $C$. The line $B B^{\prime}$ defines right angle triangles $A B B^{\prime}$ and $S B B^{\prime}$. The second arm is not shown but has angle $\theta+90^{\circ}$ to $\mathbf{v}$. Here $\mathbf{v}$ is in the plane of the interferometer for simplicity, and the azimuth angle $\psi=0$.

then

$$
t_{A B C}=t_{A B}+t_{B C}=\frac{\sqrt{4 v^{2} \gamma^{-2} L^{2} \cos ^{2}(\theta)+4 L^{2}\left(1-\frac{v^{2}}{c^{2}} \cos ^{2}(\theta)\right)\left(V^{2}-v^{2}\right)}}{\left(V^{2}-v^{2}\right)} .
$$

The corresponding travel time $t_{A B C}^{\prime}$ for the orthogonal arm is obtained from (87) by the substitution $\cos (\theta) \rightarrow \cos \left(\theta+90^{0}\right)=-\sin (\theta)$. The difference in travel times between the two arms is then $\Delta t=t_{A B C}-t_{A B C}^{\prime}$. Now trivially $\Delta t=0$ if $v=0$, but also $\Delta t=0$ when $v \neq 0$ but only if $V=c$. This then would result in a null result on rotating the apparatus. Hence the null result of Michelson interferometer experiments in the new physics is only for the special case of photons travelling in vacuum for which $V=c$. However if the interferometer is immersed in a gas then $V<c$ and a non-null effect is expected on rotating the apparatus, since now $\Delta t \neq 0$. It is essential then in analysing data to correct for this refractive index effect. The above $\Delta t$ is the change in travel time when one arm is moved through angle $\theta$. The interferometer operates by comparing the change in the difference of the travel times between the arms, and this introduces a factor of 2. Then for $V=c / n$ we find for $v<<V$ that

$$
\Delta t=\operatorname{Ln}\left(n^{2}-1\right) \frac{v^{2}}{c^{3}} \cos (2 \theta)+\mathrm{O}\left(v^{4}\right)
$$

that is $k^{2}=n\left(n^{2}-1\right)$, which gives $k=0$ for vacuum experiments $(n=1)$. So the Miller phenomenological parameter $k$ is seen to accommodate both the Fitzgerald-Lorentz con- 
traction effect and the dielectric effect, at least for gases ${ }^{8}$. This is very fortunate since being a multiplicative parameter a re-scaling of old analyses is all that is required. $\Delta t$ is non-zero when $n \neq 1$ because the refractive index effect results in incomplete cancellation of the geometrical effect and the Fitzgerald-Lorentz contraction effect. Of course it was this cancellation effect that Fitzgerald and Lorentz actually used to arrive at the length contraction hypothesis, but they failed to take the next step and note that the cancellation would be incomplete in the air operated Michelson-Morley experiment. In a bizarre development modern Michelson interferometer experiments, which use resonant cavities rather than interference effects, but for which the analysis here is easily adapted, and with the same consequences, are operated in vacuum mode. That denies these experiments the opportunity to see absolute motion effects. Nevertheless the experimentalists continue to misinterpret their null results as evidence against absolute motion. Of course these experiments are therefore restricted to merely checking the Fitzgerald-Lorentz contraction effect, and this is itself of some interest.

All data from gas-mode interferometer experiments, except for that of Miller, have been incorrectly analysed using only the first effect as in Michelson's initial theoretical treatment, and so the consequences of the other two effects have been absent. Repeating the above analysis without these two effects we arrive at the Newtonian-physics time difference which, for $v<<V$, is

$$
\Delta t=\operatorname{Ln}^{3} \frac{v^{2}}{c^{3}} \cos (2 \theta)+\mathrm{O}\left(v^{4}\right)
$$

that is $k^{2}=n^{3}$. The value of $\Delta t$, which is typically of order $10^{-17} s$ in gas-mode interferometers corresponding to a fractional fringe shift, is deduced from analysing the fringe shifts, and then the speed $v_{M}$ has been extracted using (89), instead of the correct form (88) or more generally (86). However it is very easy to correct for this oversight. From (88) and (89) we obtain for the corrected absolute (projected) speed $v_{P}$ through space, and for $n \approx 1^{+}$,

$$
v_{P}=\frac{v_{M}}{\sqrt{n^{2}-1}}
$$

For air the correction factor in (90) is significant, and even more so for Helium.

\subsection{The Michelson-Morley Experiment: 1887}

Michelson and Morley reported that their interferometer experiment in 1887 gave a 'null-result' which since then, with rare exceptions, has been claimed to support the

\footnotetext{
${ }^{8}$ For Michelson interferometers using light propagation through solids such as plastic or optical fibres there is evidence, discussed in Sect.12.2, that another effect comes into operation, namely a non-isotropic change of refractive index that causes absolute motion effects to be completely cancelled.
} 


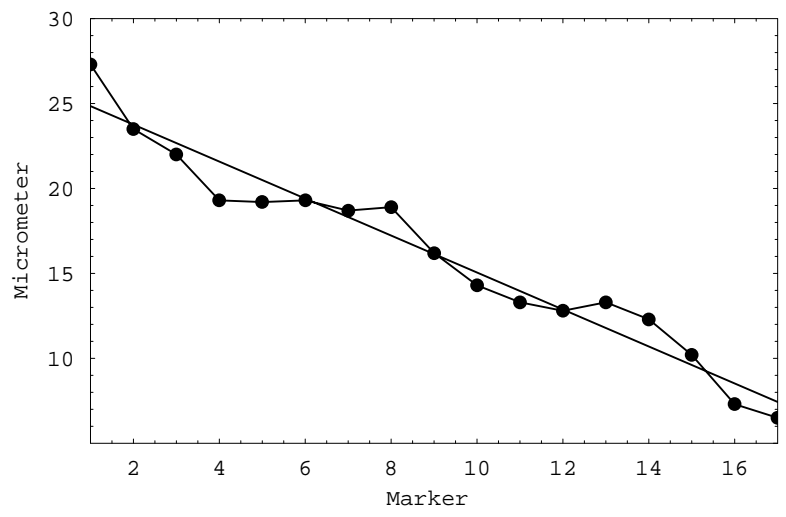

Figure 13: Plot of micrometer readings for July 11 12:00 hr (7:00 ST) showing the absolute motion induced fringe shifts superimposed on the uniform temperature induced fringe drift. Most physics books incorrectly claim that such fringe shifts were never seen by Michelson and Morley.

Einstein assumption that absolute motion has no meaning. However to the contrary the Michelson-Morley published data [88] shows non-null effects, but much smaller than they expected. They made observations of thirty-six $360^{\circ}$ turns using an $L=11$ meter length interferometer operating in air in Cleveland (Latitude $41^{0} 30^{\prime} \mathrm{N}$ ) with six turns near $12: 00$ hrs (7:00 hrs ST) on each day of July 8, 9 and 11, 1887 and similarly near 18:00 hrs (13:00 hrs ST) on July 8, 9 and 12, 1887. Each turn took approximately 6 minutes as the interferometer slowly rotated floating on a tank of mercury. They published and analysed the average of each of the 6 data sets. The fringe shifts were extremely small but within their observational capabilities.

\begin{tabular}{|c|c|c|c|c|c|c|c|c|c|}
\hline local & 16 & 1 & 2 & 3 & 4 & 5 & 6 & 7 & \\
time & 8 & 9 & 10 & 11 & 12 & 13 & 14 & 15 & 16 \\
\hline 12:00hr & 27.3 & 23.5 & 22.0 & 19.3 & 19.2 & 19.3 & 18.7 & 18.9 & \\
July 11 & 16.2 & 14.3 & 13.3 & 12.8 & 13.3 & 12.3 & 10.2 & 7.3 & 6.5 \\
\hline 18:00hr & 26.0 & 26.0 & 28.2 & 29.2 & 31.5 & 32.0 & 31.3 & 31.7 & \\
July 9 & 33.0 & 35.8 & 36.5 & 37.3 & 38.8 & 41.0 & 42.7 & 43.7 & 44.0 \\
\hline
\end{tabular}

Table 2. Examples of Michelson-Morley fringe-shift micrometer readings from [88]. The readings for July 11 12:00 hr are plotted in Fig.13.

Table 2 shows examples of the averaged fringe shift micrometer readings every $22.5^{0}$ of rotation of the Michelson-Morley interferometer [88] for July 11 12:00 hr local time and also for July 9 18:00 hr local time. The orientation of the stone slab base is indicated 
by the marks $16,1,2, \ldots$ North is mark 16 . The dominant effect was a uniform fringe drift caused by temporal temperature effects on the length of the arms, and imposed upon that are the fringe shifts corresponding to the effects of absolute motion, as shown in Fig.13.

This temperature effect can be removed by subtracting from the data in each case a best fit to the data of $a+b k,\{k=0,1,2, . ., 8\}$ for the first $0^{0}$ to $180^{0}$ part of each rotation data set. Then multiplying by 0.02 for the micrometer thread calibration gives the fringe-shift data points in Fig.15. This factor of 0.02 converts the micrometer readings to fringe shifts expressed as fractions of a wavelength. Similarly a linear fit has been made to the data from the $180^{\circ}$ to $360^{\circ}$ part of each rotation data set. Separating the full $360^{0}$ rotation into two $180^{\circ}$ parts reduces the effect of the temperature drift not being perfectly linear in time.

In the new physics there are four main velocities that contribute to the total velocity:

$$
\mathbf{v}=\mathbf{v}_{\text {cosmic }}+\mathbf{v}_{\text {tangent }}-\mathbf{v}_{\text {in }}-\mathbf{v}_{E}
$$

Here $\mathbf{v}_{\text {cosmic }}$ is the velocity of the solar system relative to some cosmologically defined galactic quantum-foam system (discussed later) while the other three are local effects: (i) $\mathbf{v}_{\text {tangent }}$ is the tangential orbital velocity of the earth about the sun, (ii) $\mathbf{v}_{\text {in }}$ is a quantum-gravity radial in-flow of the quantum foam past the earth towards the sun, and (iii) the corresponding quantum-foam in-flow into the earth is $\mathbf{v}_{E}$ and makes no contribution to a horizontally operated interferometer, assuming the velocity superposition approximation ${ }^{9}$ of Sect.9.7. The minus signs in (91) arise because, for example, the in-flow towards the sun requires the earth to have an outward directed velocity against that in-flow in order to maintain a fixed distance from the sun, as shown in Fig.14. For circular orbits and using in-flow form of Newtonian gravity the speeds $v_{\text {tangent }}$ and $v_{\text {in }}$ are given by

$$
\begin{gathered}
v_{\text {tangent }}=\sqrt{\frac{G_{N} M}{R}}, \\
v_{i n}=\sqrt{\frac{2 G_{N} M}{R}},
\end{gathered}
$$

while the net speed $v_{R}$ of the earth from the vector sum $\mathbf{v}_{R}=\mathbf{v}_{\text {tangent }}-\mathbf{v}_{\text {in }}$ is

$$
v_{R}=\sqrt{\frac{3 G_{N} M}{R}},
$$

where $M$ is the mass of the sun, $R$ is the distance of the earth from the sun, and $G_{N}$ is Newton's gravitational constant. $G_{N}$ is essentially a measure of the rate at which matter

\footnotetext{
${ }^{9}$ and also that the turbulence associated with that flow is not significant.
} 
effectively 'dissipates' the quantum-foam. The gravitational acceleration arises from inhomogeneities in the flow. These expressions give $v_{\text {tangent }}=30 \mathrm{~km} / \mathrm{s}, v_{\text {in }}=42.4 \mathrm{~km} / \mathrm{s}$ and $v_{R}=52 \mathrm{~km} / \mathrm{s}$.

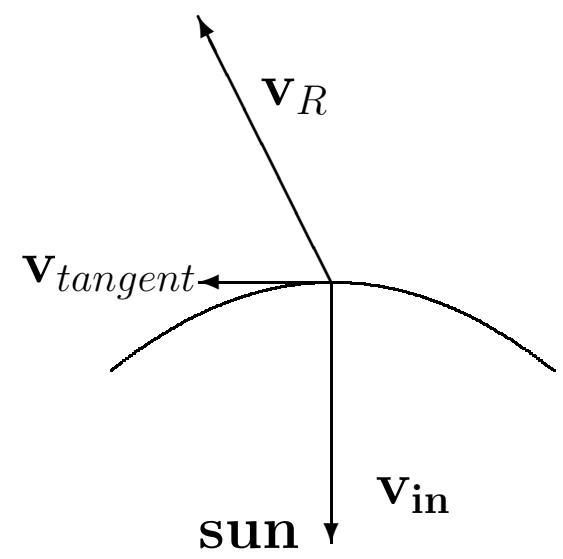

Figure 14: Orbit of earth about the sun defining the plane of the ecliptic with tangential orbital velocity $\mathbf{v}_{\text {tangent }}$ and quantum-foam in-flow velocity $\mathbf{v}_{\text {in }}$. Then $\mathbf{v}_{R}=\mathbf{v}_{\text {tangent }}-\mathbf{v}_{\text {in }}$ is the velocity of the earth relative to the quantum foam, after subtracting $\mathbf{v}_{\text {cosmic }}$.

Fig.15 shows all the data for the 1887 Michelson-Morley experiment for the fringe shifts after removal of the temperature drift effect for each averaged 180 degree rotation. The dotted curves come from the best fit of $\frac{0.4}{30^{2}} k_{\text {air }}^{2} v_{P}^{2} \cos (2(\theta-\psi))$ to the data. The coefficient $0.4 / 30^{2}$ arises as the apparatus would give a 0.4 fringe shift, as a fraction of a wavelength, with $k=1$ if $v_{P}=30 \mathrm{~km} / \mathrm{s}$ [88]. Shown in each figure is the resulting value of $v_{P}$. In some cases the data does not have the expected $\cos (2(\theta-\psi))$ form, and so the corresponding values for $v_{P}$ are not meaningful. The remaining fits give $v_{P}=331 \pm 30$ $\mathrm{km} / \mathrm{s}$ for the $7: 00 \mathrm{hr}(\mathrm{ST})$ data, and $v_{P}=328 \pm 50 \mathrm{~km} / \mathrm{s}$ for the $13: 00 \mathrm{hr}(\mathrm{ST})$ data. For comparison the full curves show the predicted form for the Michelson-Morley data, computed for the latitude of Cleveland, using the Miller direction (see later) for $\mathbf{v}_{\text {cosmic }}$ of Right Ascension and Declination $\left(\alpha=4^{h r} 54^{\prime}, \delta=-70^{0} 30^{\prime}\right)$ and incorporating the tangential and in-flow velocity effects for July. The magnitude of the theoretical curves are in general in good agreement with the magnitudes of the experimental data, excluding those cases where the data does not have the sinusoidal form. However there are significant fluctuations in the azimuth angle. These fluctuations are also present in the Miller data, and together suggest that this is a real physical phenomenon, and not solely due to difficulties with the operation of the interferometer.

The Michelson-Morley interferometer data clearly shows the characteristic sinusoidal form with period $180^{\circ}$ together with a large speed. Ignoring the effect of the refractive 

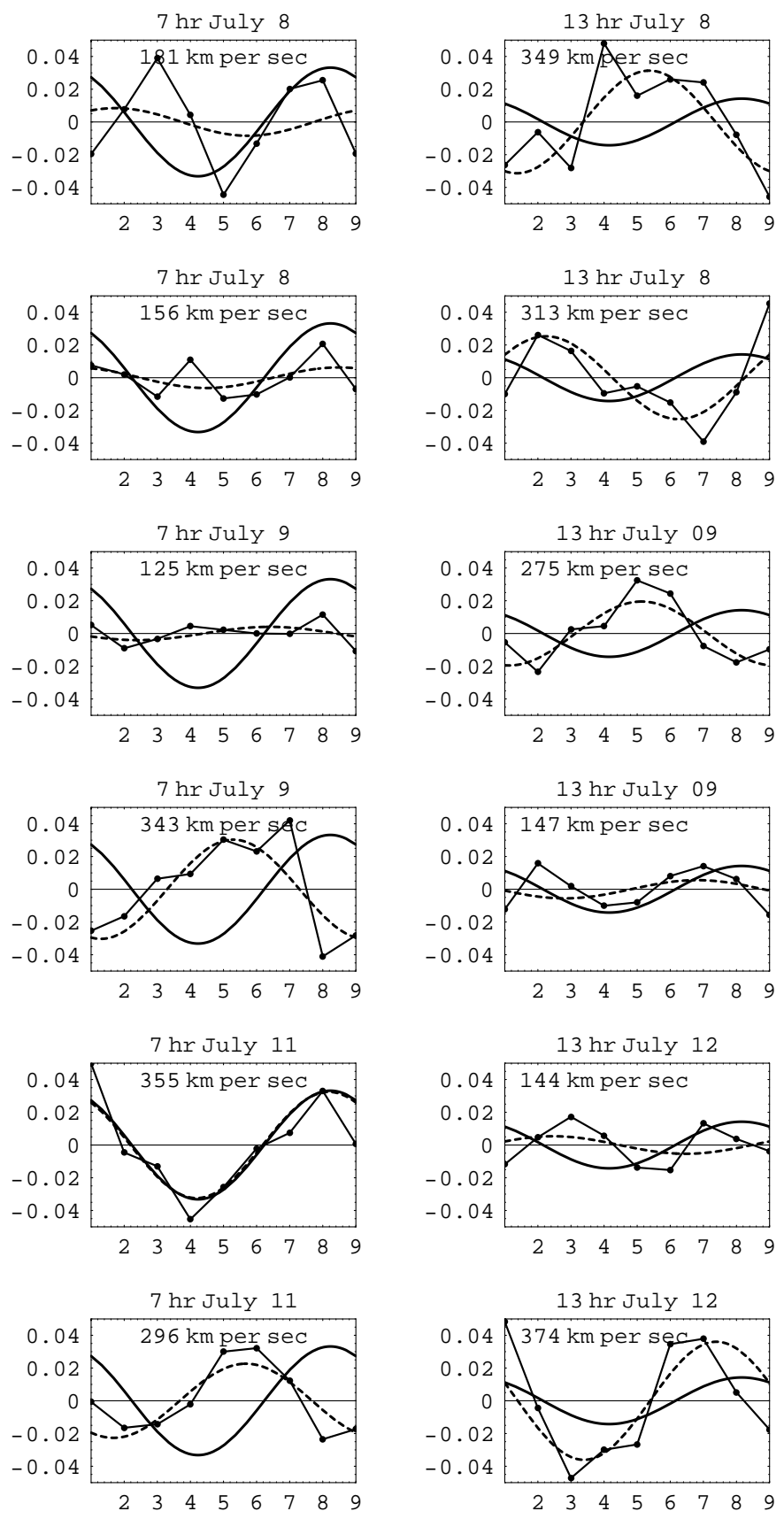

Figure 15: Shows all the Michelson-Morley 1887 data after removal of the temperature induced fringe drifts. The data for each $360^{\circ}$ full turn (the average of 6 individual turns) is divided into the 1st and 2 nd $180^{\circ}$ parts and plotted one above the other. The dotted curve shows a best fit to the data, while the full curves show the expected forms using the Miller direction for $\mathbf{v}_{\text {cosmic }}$. 


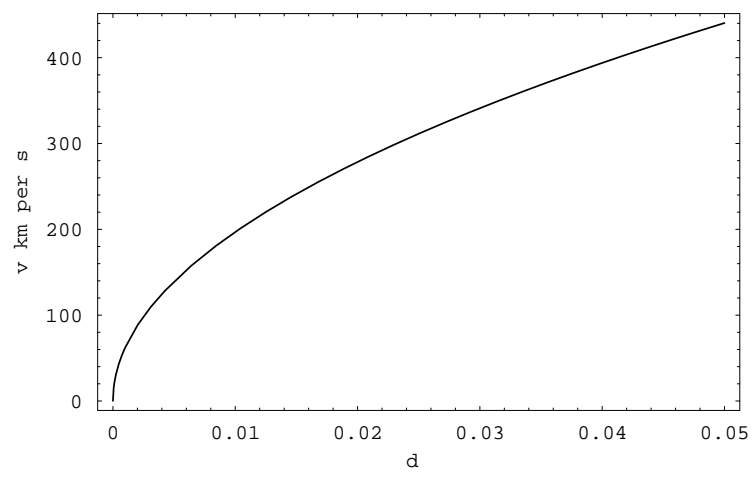

Figure 16: Speed calibration for Michelson-Morley experiment. This shows the value of $v_{P}$ in $\mathrm{km} / \mathrm{s}$ for values of the fringe shifts, $d$, expressed as a fraction of one wavelength of the light used, as shown in Fig.15

index, namely using the Newtonian value of $k=1$, gives speeds reduced by the factor $k_{\text {air }}$, namely $k_{\text {air }} v_{P}=0.0241 \times 330 \mathrm{~km} / \mathrm{s}=7.9 \mathrm{~km} / \mathrm{s}$. Michelson and Morley reported speeds in the range $5 \mathrm{~km} / \mathrm{s}-7.5 \mathrm{~km} / \mathrm{s}$. These slightly smaller speeds arise because they averaged all the 7:00 $\mathrm{hr}$ (ST) data, and separately all the 13:00 hr (ST) data, whereas here some of the lower quality data have not been used. Michelson was led to the false conclusion that because this speed of some $8 \mathrm{~km} / \mathrm{s}$ was considerably less than the orbital speed of $30 \mathrm{~km} / \mathrm{s}$ the interferometer must have failed to have detected absolute motion, and that the data was merely caused by experimental imperfections. This was the flawed analysis that led to the incorrect conclusion by Michelson and Morley that the experiment had failed to detect absolute motion. The consequences for physics were extremely damaging, and are only now being rectified after some 115 years.

\subsection{The Miller Interferometer Experiment: 1925-1926}

Dayton Miller developed and operated a Michelson interferometer for over twenty years, see Fig.17, with the main sequence of observations being on Mt.Wilson in the years 1925-1926, with the results reported in 1933 by Miller [89]. Accounts of the Miller experiments are available in Swenson [97] and DeMeo ${ }^{10}$ [98]. Miller developed his huge interferometer over the years, from 1902 to 1906, in collaboration with Morley, and later at Mt.Wilson where the most extensive interferometer observations were carried out. Miller was meticulous in perfecting the operation of the interferometer and performed

\footnotetext{
${ }^{10}$ Note however that DeMeo interprets Miller's 'small' speeds, that is some $10 \mathrm{~km} / \mathrm{s}$, as being a result of entrainment, and not as herein a consequence of the misunderstanding of the physics involved in the operation of the interferometer.
} 


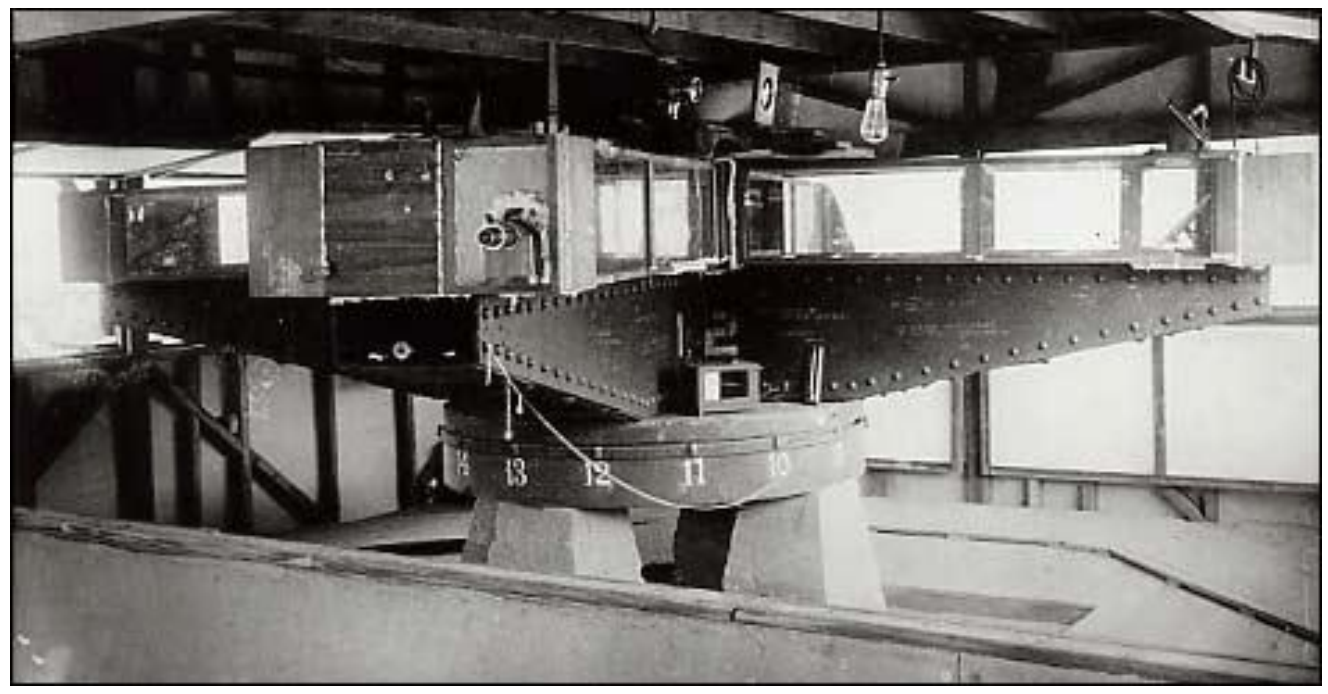

Figure 17: Miller's interferometer with an effective arm length of $L=32 \mathrm{~m}$ achieved by multiple reflections, as shown in Fig.18. Used by Miller on Mt.Wilson to perform the 1925-1926 observations of absolute motion. The steel arms weighed 1200 kilograms and floated in a tank of 275 kilograms of Mercury. From Case Western Reserve University Archives.

many control experiments. The biggest problem to be controlled was the effect of temperature changes on the lengths of the arms. It was essential that the temperature effects were kept as small as possible, but so long as each turn was performed sufficiently quickly, any temperature effect could be assumed to have been linear with respect to the angle of rotation. Then a uniform background fringe drift could be removed, as in the Michelson-Morley data analysis (see Fig.13).

In all some 200,000 readings were taken during some 12,000 turns of the interferometer $^{11}$. Analysis of the data requires the extraction of the speed $v_{M}$ and the azimuth angle $\psi$ by effectively fitting the observed time differences, obtained from the observed fringe shifts, using (86), but with $k=1$. Miller was of course unaware of the full theory of the interferometer and so he assumed the Newtonian theory, which neglected both the Fitzgerald-Lorentz contraction and air effects.

Miller performed this analysis of his data by hand, and the results for April, August and September 1925 and February 1926 are shown in Fig.19. The speeds shown are

\footnotetext{
${ }^{11}$ In a remarkable development in 2002 as a result of a visit by James DeMeo to Case Western Reserve University the original Miller data was located, some 61 years after Miller's death in 1941. Until then it was thought that the data had been destroyed. Analysis of that data by the author of this article has confirmed the accuracy of Miller's analysis. Using more thorough computer based techniques the data is now being re-analysed
} 


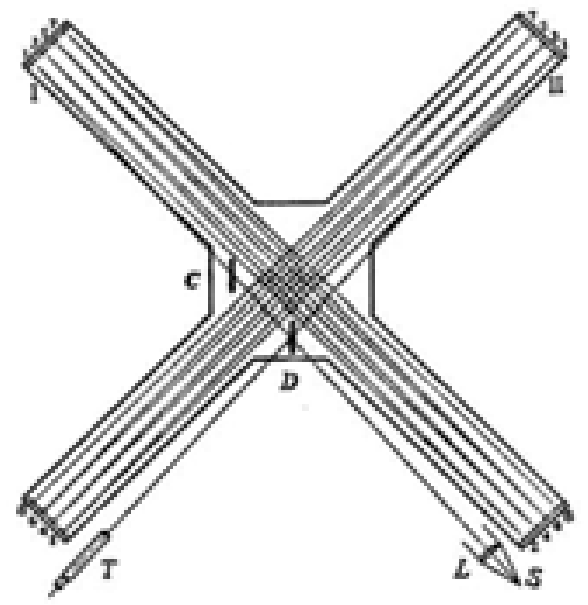

Figure 18: The effective arm length of $L=32 \mathrm{~m}$ was achieved by multiple reflections.

the Michelson speeds $v_{M}$, and these are easily corrected for the two neglected effects by dividing these $v_{M}$ by $k_{\text {air }}=\sqrt{ }\left(n^{2}-1\right)=0.0241$, as in (90). Then for example a speed of $v_{M}=10 \mathrm{~km} / \mathrm{s}$ gives $v_{P}=v_{M} / k_{\text {air }}=415 \mathrm{~km} / \mathrm{s}$. However this correction procedure was not available to Miller. He understood that the theory of the Michelson interferometer was not complete, and so he introduced the phenomenological parameter $k$ in (86). We shall denote his values by $\bar{k}$. Miller noted, in fact, that $\bar{k}^{2}<<1$, as we would now expect. Miller then proceeded on the assumption that $\mathbf{v}$ should have only two components: (i) a cosmic velocity of the solar system through space, and (ii) the orbital velocity of the earth about the sun. Over a year this vector sum would result in a changing $\mathbf{v}$, as was in fact observed, see Fig.19. Further, since the orbital speed was known, Miller was able to extract from the data the magnitude and direction of $\mathbf{v}$ as the orbital speed offered an absolute scale. For example the dip in the $v_{M}$ plots for sidereal times $\tau \approx 16^{h r}$ is a clear indication of the direction of $\mathbf{v}$, as the dip arises at those sidereal times when the projection $v_{P}$ of $\mathbf{v}$ onto the plane of the interferometer is at a minimum. During a $24 \mathrm{hr}$ period the value of $v_{P}$ varies due to the earth's rotation. As well the $v_{M}$ plots vary throughout the year because the vectorial sum of the earth's orbital velocity $\mathbf{v}_{\text {tangent }}$ and the cosmic velocity $\mathbf{v}_{\text {cosmic }}$ changes. There are two effects here as the direction of $\mathbf{v}_{\text {tangent }}$ is determined by both the yearly progression of the earth in its orbit about the sun, and also because the plane of the ecliptic is inclined at $23.5^{0}$ to the celestial plane. Figs.21 and 23 show the expected theoretical variation of both $v_{P}$ and the azimuth $\psi$ during one sidereal day in the months of April, August, September and February. These plots show the clear signature of absolute motion effects as seen in the actual interferometer data of Fig.19.

Note that the above corrected Miller projected absolute speed of approximately $v_{P}=$ 

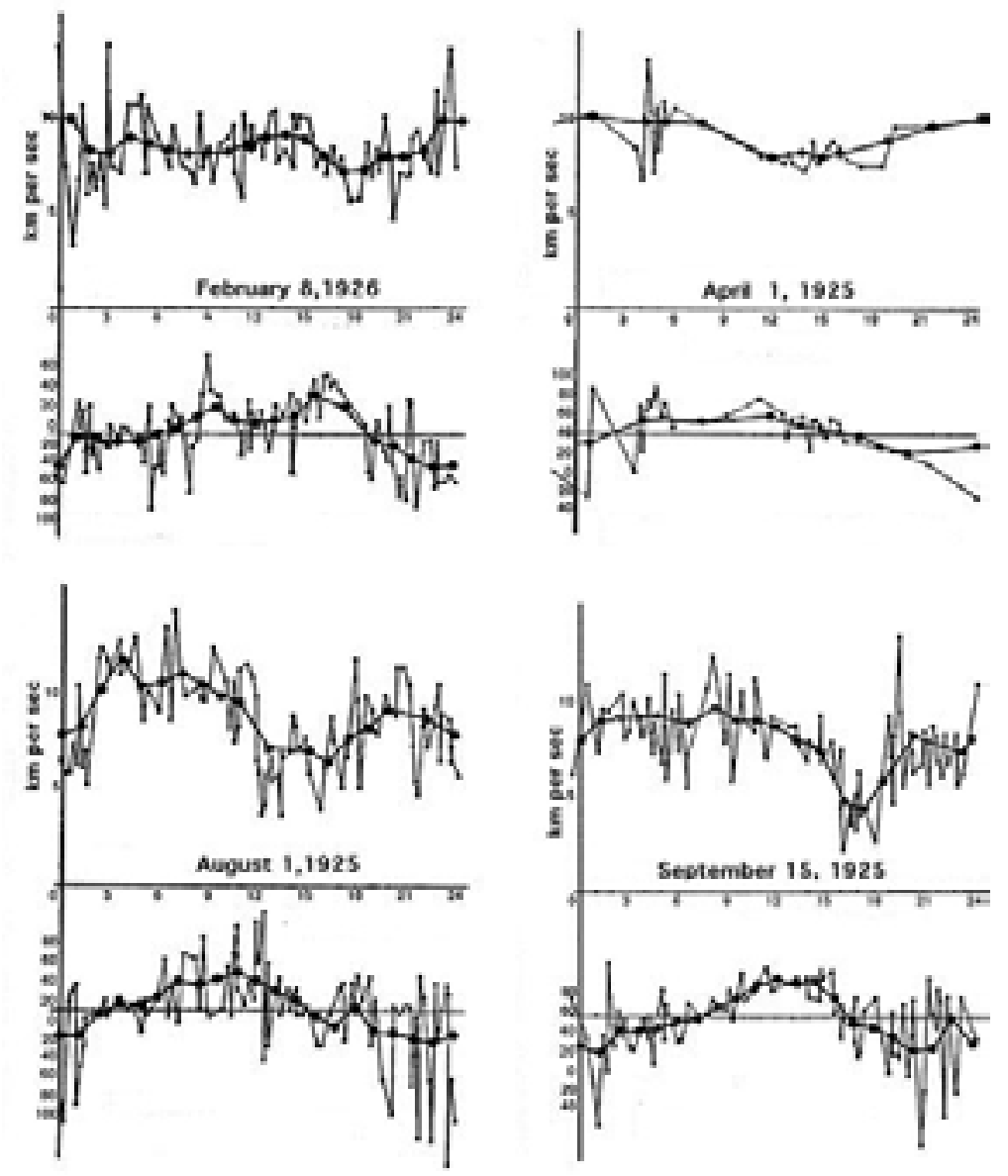

Figure 19: Miller's results from the 1925-1926 observations of absolute motion showing the projected 'Michelson' speed $v_{M}$ in $\mathrm{km} / \mathrm{s}$ and azimuth angle $\psi$ in degrees plotted against sidereal time in hours. The results are for April, August and September 1925 and February 1926. In most cases the results arise from observations extending over much of each month, i.e not from a single day in each month. Therefore the data points are not strictly in chronological order. The lines joining the data points are merely to make the data points clearer. The smoother line is a running time average computed by Miller. The fluctuations in both $v_{M}$ and $\psi$ appear to be a combination of apparatus effects and genuine physical phenomena caused by turbulence in the gravitational in-flow of space towards the sun. Each data point arises from analysis of the average of twenty full rotations of the interferometer. 

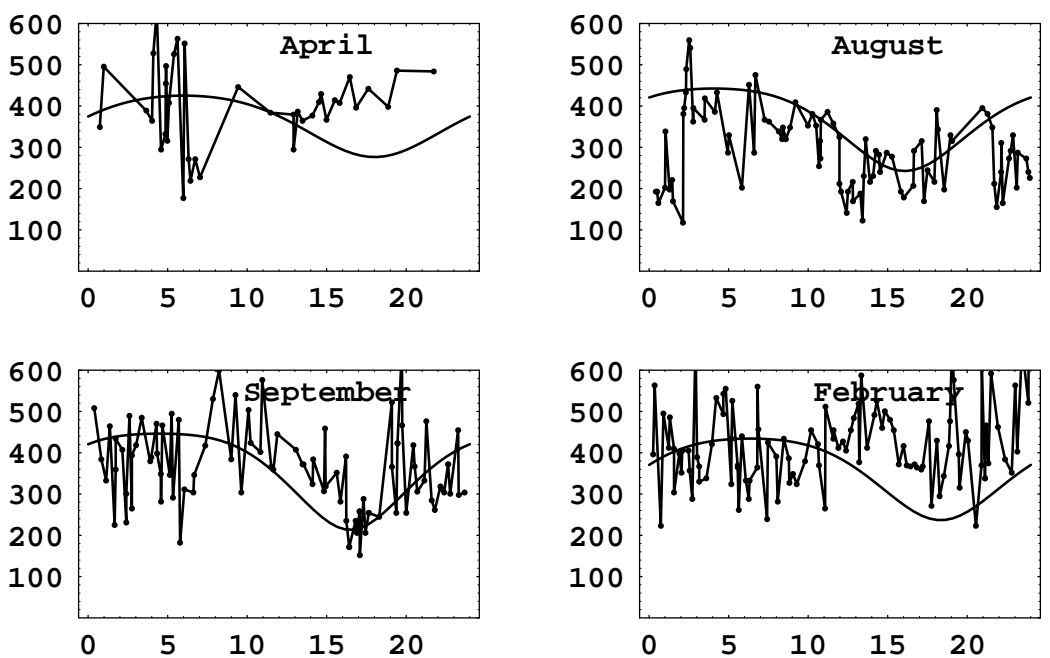

Figure 20: Miller interferometer projected speeds $v_{P}$ in $\mathrm{km} / \mathrm{s}$ showing both data and best fit of theory giving $v_{\text {cosmic }}=433 \mathrm{~km} / \mathrm{s}$ in the direction $\left(\alpha=5.2^{h r}, \delta=\right.$ $\left.-67^{0}\right)$, and using $n=1.000226$ appropriate for the altitude of Mt. Wilson

$415 \mathrm{~km} / \mathrm{s}$ is completely consistent with the corrected projected absolute speed of some $330 \mathrm{~km} / \mathrm{s}$ from the Michelson-Morley experiment, though neither Michelson nor Miller were able to apply this correction. The difference in magnitude is completely explained by Cleveland having a higher latitude than Mt. Wilson, and also by the only two sidereal times of the Michelson-Morley observations. So from his 1925-1926 observations Miller had completely confirmed the true validity of the Michelson-Morley observations and was able to conclude, contrary to their published conclusions, that the 1887 experiment had in fact detected absolute motion. But it was too late. By then the physicists had incorrectly come to believe that absolute motion was inconsistent with various 'relativistic effects' that had by then been observed. This was because the Einstein formalism had been 'derived' from the assumption that absolute motion was without meaning and so unobservable in principle. Of course the earlier interpretation of relativistic effects by Lorentz had by then lost out to the Einstein interpretation.

\subsection{The Illingworth Experiment: 1927}

In 1927 Illingworth [91] performed a Michelson interferometer experiment in which the light beams passed through the gas Helium,

... as it has such a low index of refraction that variations due to temperature changes are reduced to a negligible quantity. 


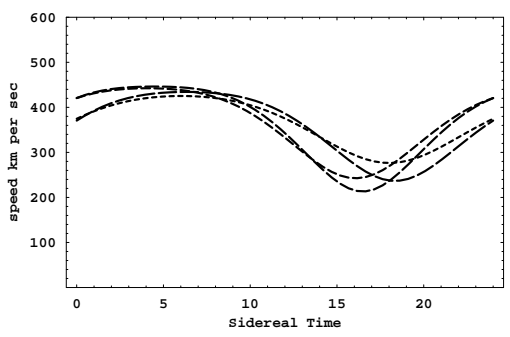

Figure 21: Expected theoretical variation of the projected velocity $v_{P}$ during one sidereal day in the months of April, August, September and February, labelled by increasing dash length for cosmic speed of $433 \mathrm{~km} / \mathrm{s}$ in the direction $\left(\alpha=5.2^{h r}, \delta=-67^{0}\right)$. This shows how the signature of the effect changes over a year.

For Helium at STP $n=1.000036$ and so $k_{H e}^{2}=0.00007$, which results in an enormous reduction in sensitivity of the interferometer. Nevertheless this experiment gives an excellent opportunity to check the $n$ dependence in (90). Illingworth, not surprisingly, reported no "ether drift to an accuracy of about one kilometer per second". Múnera [100] re-analysed the Illingworth data to obtain a speed $v_{M}=3.13 \pm 1.04 \mathrm{~km} / \mathrm{s}$. The correction factor in (90), $1 / \sqrt{n_{H e}^{2}-1}=118$, is large for Helium and gives $v=368 \pm 123 \mathrm{~km} / \mathrm{s}$. As shown in Fig.24 the Illingworth observations now agree with those of MichelsonMorley and Miller, though they would certainly be inconsistent without the $n$-dependent correction, as shown in the lower data points (shown at $5 \times$ scale).

So the use by Illingworth of Helium gas has turned out have offered a fortuitous opportunity to confirm the validity of the refractive index effect, though because of the insensitivity of this experiment the resulting error range is significantly larger than those of the other interferometer observations. So finally it is seen that the Illingworth experiment detected absolute motion with a speed consistent with all other observations.

\subsection{The Joos Experiment: 1930}

Joos set out to construct and operate a large vacuum Michelson interferometer at the Zeiss Works in Jena, Germany [92] in 1930. This interferometer had an effective arm length of $10 \mathrm{~m}$ achieved using multiple reflections in each arm. The vacuum sealing was ineffective and the penetration of air into the vacuum vessel caused problematic vibrations. Subsequently Joos used helium, assuming apparently that helium could be considered as a substitute for a true vacuum ${ }^{12}$. The use of helium is not mentioned in the Joos paper [92], but is mentioned by Swenson [97]. Joos recorded the fringe

\footnotetext{
${ }^{12}$ Thanks to Dr Lance McCarthy for pointing out the use of helium in this experiment and in extracting the data from the Joos paper.
} 

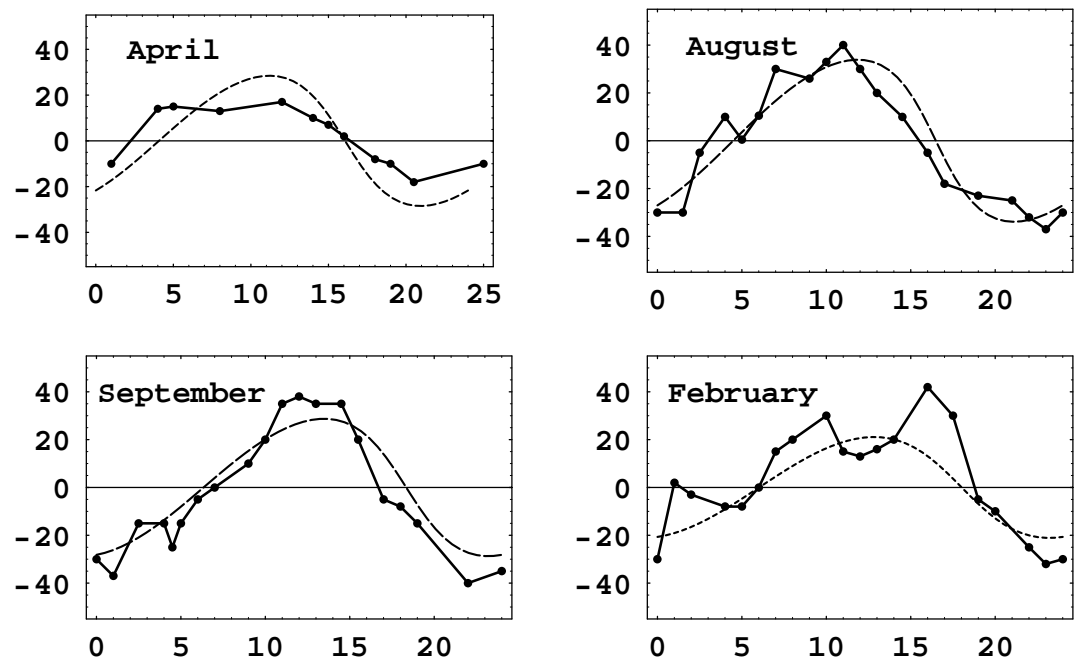

Figure 22: Miller azimuths $\psi$, measured from south, showing both data and best fit of theory giving $v_{\text {cosmic }}=433 \mathrm{~km} / \mathrm{s}$ in the direction $\left(\alpha=5.2^{h r}, \delta=-67^{0}\right)$, and using $n=1.000226$ appropriate for the altitude of Mt. Wilson

shifts photographically, and subsequently analysed the images using a photometer. The data for 22 rotations throughout the day of May 30, 1930 are shown in Fig.25, and are reproduced from Fig.11 of [92]. From that data Joos concluded, using an analysis that

did not take account of the special relativistic length contraction effect, that the fringe shifts corresponded to a speed of only $1.5 \mathrm{~km} / \mathrm{s}$. However as previously noted such an analysis is completely flawed. As well the data in Fig. 25 shows that for all but one of the rotations the fringe shifts were poorly recorded. Only in the one rotation, at $1123^{58}$, does the data actually look like the form expected. This is probably not accidental as the maximum fringe shift was expected at that time, based on the Miller direction of absolute motion. In Fig.26 data from that one rotation are compared with the form expected for Jena on May 30 using the Miller speed and direction together with the new refractive index effect,and using the refractive index of helium. The agreement is quite remarkable. So again contrary the Joos paper and to subsequent history, Joos did in fact detect a very large velocity of absolute motion.

\subsection{The New Bedford Experiment: 1963}

In 1964 from an absolute motion detector experiment at New Bedford, latitude $42^{0} \mathrm{~N}$, Jaseja et al. [102] reported yet another 'null result'. In this experiment two He-Ne masers were mounted with axes perpendicular on a rotating table, see Fig.27. Rotation 


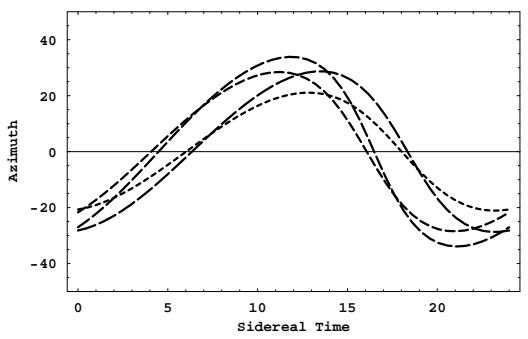

Figure 23: Expected theoretical variation of the azimuths $\psi$, measured from south, during one sidereal day in the months of April, August, September and February, labelled by increasing dash length, for a cosmic speed of $433 \mathrm{~km} / \mathrm{s}$ in the direction $\left(\alpha=5.2^{h r}, \delta=\right.$ $\left.-67^{0}\right)$. This shows how the signature of the effect changes over a year.

of the table through $90^{\circ}$ produced repeatable variations in the frequency difference of about $275 \mathrm{kHz}$, an effect attributed to magnetorestriction in the Invar spacers due to the earth's magnetic field. Observations over some six consecutive hours on January 20, 1963 from 6:00 am to 12:00 noon local time did produce a 'dip' in the frequency difference of some $3 \mathrm{kHz}$ superimposed on the $275 \mathrm{kHz}$ effect, as shown in Fig. 28 in which the local times have been converted to sidereal times. The most noticeable feature is that the dip occurs at approximately $17-18: 00^{h r}$ sidereal time (or $9-10: 00 \mathrm{hrs}$ local time), which agrees with the direction of absolute motion observed by Miller and also by DeWitte (see Sect.10.8). It was most fortunate that this particular time period was chosen as at other times the effect is much smaller, as shown for the February data in Fig.19 which shows the minimum at 18:00 ${ }^{h r}$ sidereal time. The local times were chosen by Jaseja et al. such that if the only motion was due to the earth's orbital speed the maximum frequency difference, on rotation, should have occurred at 12:00hr local time, and the minimum frequency difference at 6:00 hr local time, whereas in fact the minimum frequency difference occurred at 9:00 hr local time.

As for the Michelson-Morley experiment the analysis of the New Bedford experiment was also bungled. Again this apparatus can only detect the effects of absolute motion if the cancellation between the geometrical effects and Fitzgerald-Lorentz length contraction effects is incomplete. This occurs only when the radiation travels in a gas, here the He-Ne gas present in the maser.

This double maser apparatus is essentially equivalent to a Michelson interferometer, and so the simple analysis of Sect.16.3 is appropriate. Then the resonant frequency $\nu$ of each maser is proportional to the reciprocal of the out-and-back travel time. 


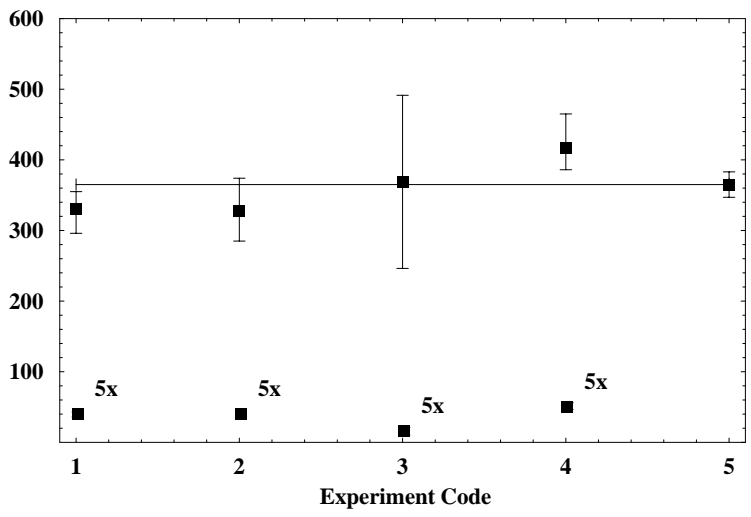

Figure 24: Speeds $v$ in $\mathrm{km} / \mathrm{s}$ determined from various Michelson interferometer experiments (1)-(4) and CMB (5): (1) Michelson-Morley (noon observations) and (2) $\left(18^{h}\right.$ observations) see Sect.10.3, (3) Illingworth [91], (4) Miller, Mt.Wilson [89], and finally in (5) the speed from observations of the CMB spectrum dipole term [101]. The results (1)-(3) are not corrected for the $\pm 30 \mathrm{~km} / \mathrm{s}$ of the orbital motion of the earth about the sun or for the gravitational in-flow speed, though these corrections were made for (4) with the speeds from Table 3 . The horizontal line at $v=369 \mathrm{~km} / \mathrm{s}$ is to aid comparisons with the CMB frame speed data. The Miller direction is different to the CMB direction. Due to the angle between the velocity vector and the plane of interferometer the results (1)-(3) are less than or equal to the true speed, while the result for (4) is the true speed as this projection effect was included in the analysis. These results demonstrate the remarkable consistency between the three interferometer experiments. The Miller speed agrees with the speed from the DeWitte non-interferometer experiment, in Sect.10.8. The lower data, magnified by a factor of 5 , are the original speeds $v_{M}$ determined from fringe shifts using (85) with $k=1$. This figure updates the corresponding figure in Ref.[12].

For maser 1

$$
\nu_{1}=m \frac{V^{2}-v^{2}}{2 L V \sqrt{1-\frac{v^{2}}{c^{2}}}},
$$

for which a Fitzgerald-Lorentz contraction occurs, while for maser 2

$$
\nu_{2}=m \frac{\sqrt{V^{2}-v^{2}}}{2 L} .
$$

Here $m$ refers to the mode number of the masers. When the apparatus is rotated the net observed frequency difference is $\delta \nu=2\left(\nu_{2}-\nu_{1}\right)$, where the factor of ' 2 ' arises as the roles of the two masers are reversed after a $90^{\circ}$ rotation. Putting $V=c / n$ we find for 


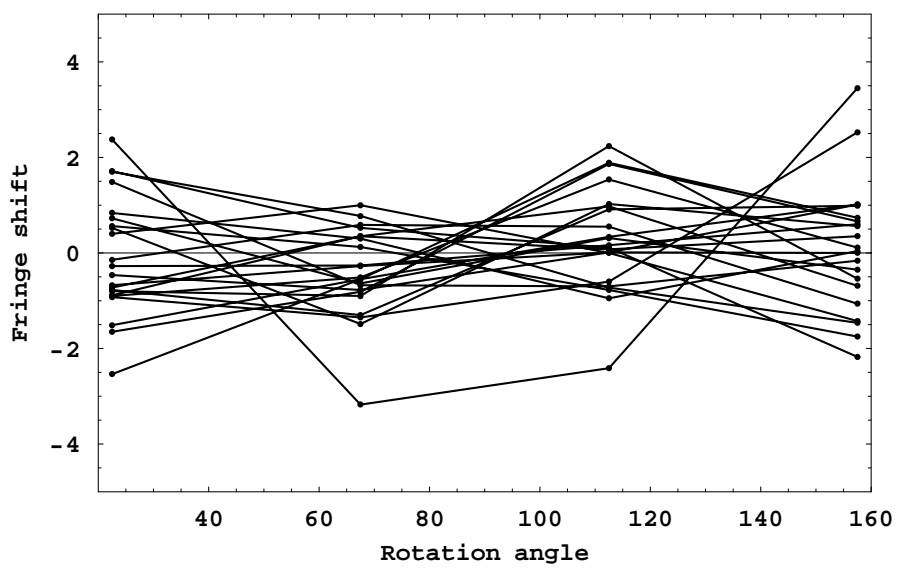

Figure 25: The Joos fringes shifts in $\lambda / 1000$ recorded on May 30, 1930 from a Michelson interferometer using helium. Only one of the rotations produced a clean signal of the form expected.

$v<<V$ and with $\nu_{0}$ the at-rest resonant frequency, that

$$
\delta \nu=\left(n^{2}-1\right) \nu_{0} \frac{v^{2}}{c^{2}}+O\left(\frac{v^{4}}{c^{4}}\right) .
$$

If we use the Newtonian physics analysis, as in Jaseja et al. [102], which neglects both the Fitzgerald-Lorentz contraction and the refractive index effect, then we obtain $\delta \nu=\nu_{0} v^{2} / c^{2}$, that is without the $n^{2}-1$ term, just as for the Newtonian analysis of the Michelson interferometer itself. Of course the very small magnitude of the absolute motion effect, which was approximately $1 / 1000$ that expected assuming only an orbital speed of $v=30 \mathrm{~km} / \mathrm{s}$ in the Newtonian analysis, occurs simply because the refractive index of the He-Ne gas is very close to one ${ }^{13}$. Nevertheless given that it is small the sidereal time of the obvious 'dip' coincides almost exactly with that of the other observations of absolute motion.

The New Bedford experiment was yet another missed opportunity to have revealed the existence of absolute motion. Again the spurious argument was that because the Newtonian physics analysis gave the wrong prediction then Einstein relativity must be correct. But the analysis simply failed to take account of the Fitzgerald-Lorentz contraction, which had been known since the end of the $19^{\text {th }}$ century, and the refractive index effect which had an even longer history. As well the authors failed to convert their local times to sidereal times and compare the time for the 'dip' with Miller's time ${ }^{14}$.

\footnotetext{
${ }^{13}$ It is possible to compare the refractive index of the He-Ne gas mixture in the maser with the value extractable from this data: $n^{2}=1+30^{2} /\left(1000 \times 400^{2}\right)$, or $n=1.0000028$.

${ }^{14}$ There is no reference to Miller's 1933 paper in Ref.[102].
} 


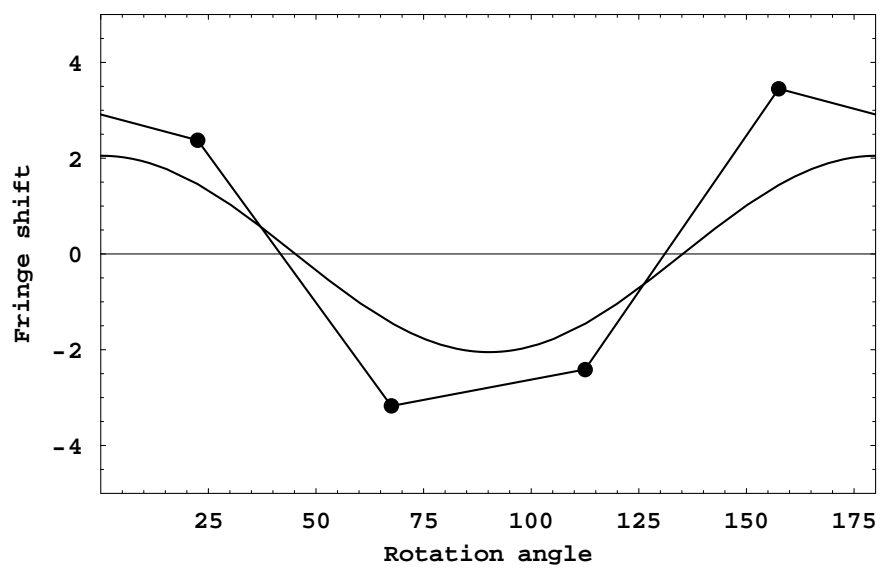

Figure 26: Comparison of the Joos data for the one good rotation at $1123^{58}$ with the theoretical prediction using the speed $v_{\text {cosmic }}=433 \mathrm{~km} / \mathrm{s}$ in the direction $(\alpha=$ $5.2^{h r}, \delta=-67^{0}$ ) from the Miller experiment, together with the length contraction and refractive index effect, here for helium. The experimental uncertainty is \pm 1 .

\subsection{The DeWitte Experiment: 1991}

The Michelson-Morley, Illingworth, Miller and New Bedford experiments all used Michelson interferometers or its equivalent in gas mode, and all revealed absolute motion. The Michelson interferometer is a 2nd-order device meaning that the time difference between the 'arms' is proportional to $(v / c)^{2}$. There is also a factor of $n^{2}-1$ and for gases like air and particularly Helium or Helium-Neon mixes this results in very small time differences and so these experiments were always very difficult. Of course without the gas the Michelson interferometer is incapable of detecting absolute motion ${ }^{15}$, and so there are fundamental limitations to the use of this interferometer in the study of absolute motion and related effects.

In a remarkable development in 1991 a research project within Belgacom, the Belgium telecommunications company, stumbled across yet another detection of absolute motion, and one which turned out to be 1st-order in $v / c$. The study was undertaken by Roland DeWitte [103]. This organisation had two sets of atomic clocks in two buildings in Brussels separated by $1.5 \mathrm{~km}$ and the research project was an investigation of the task of synchronising these two clusters of atomic clocks. To that end $5 \mathrm{MHz}$ radiofrequency signals were sent in both directions through two buried coaxial cables linking the two clusters. The atomic clocks were caesium beam atomic clocks, and there were three in each cluster. In that way the stability of the clocks could be established and monitored. One cluster was in a building on Rue du Marais and the second cluster was due south in a

\footnotetext{
${ }^{15}$ So why not use a transparent solid in place of the gas? See Sect.12.2 for the discussion.
} 
(a)

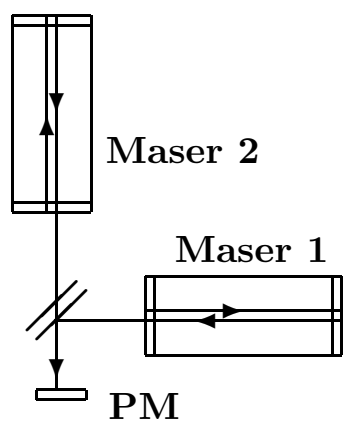

(b)

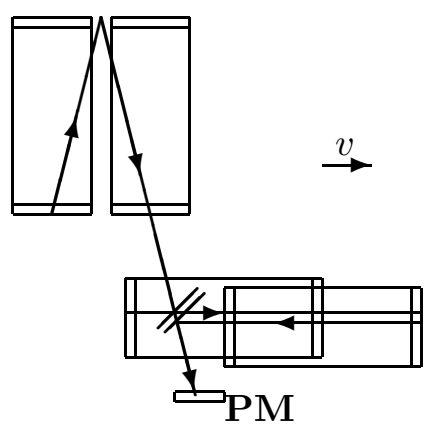

Figure 27: Schematic diagram for recording the variations in beat frequency between two optical masers: (a) when at absolute rest, (b) when in absolute motion at velocity $\mathbf{v}$. $\mathrm{PM}$ is the photomultiplier detector. The apparatus was rotated back and forth through $90^{\circ}$.

building on Rue de la Paille. Digital phase comparators were used to measure changes in times between clocks within the same cluster and also in the propagation times of the RF signals. Time differences between clocks within the same cluster showed a linear phase drift caused by the clocks not having exactly the same frequency together with short term and long term noise. However the long term drift was very linear and reproducible, and that drift could be allowed for in analysing time differences in the propagation times between the clusters.

Changes in propagation times were observed and eventually observations over 178 days were recorded. A sample of the data, plotted against sidereal time for just three days, is shown in Fig.29. DeWitte recognised that the data was evidence of absolute motion but he was unaware of the Miller experiment and did not realise that the Right Ascension for maximum/minimum propagation time agreed almost exactly with Miller's direction $(\alpha, \delta)=\left(5.2^{h},-67^{0}\right)$. In fact DeWitte expected that the direction of absolute motion should have been in the CMB direction, but that would have given the data a totally different sidereal time signature, namely the times for maximum/minimum would have been shifted by 6 hrs. The declination of the velocity observed in this DeWitte experiment cannot be determined from the data as only three days of data are available. However assuming exactly the same declination as Miller the speed observed by DeWitte appears to be also in excellent agreement with the Miller speed, which in turn is in agreement with that from the Michelson-Morley and Illingworth experiments, as shown in Fig.24.

Being 1st-order in $v / c$ the Belgacom experiment is easily analysed to sufficient accu- 


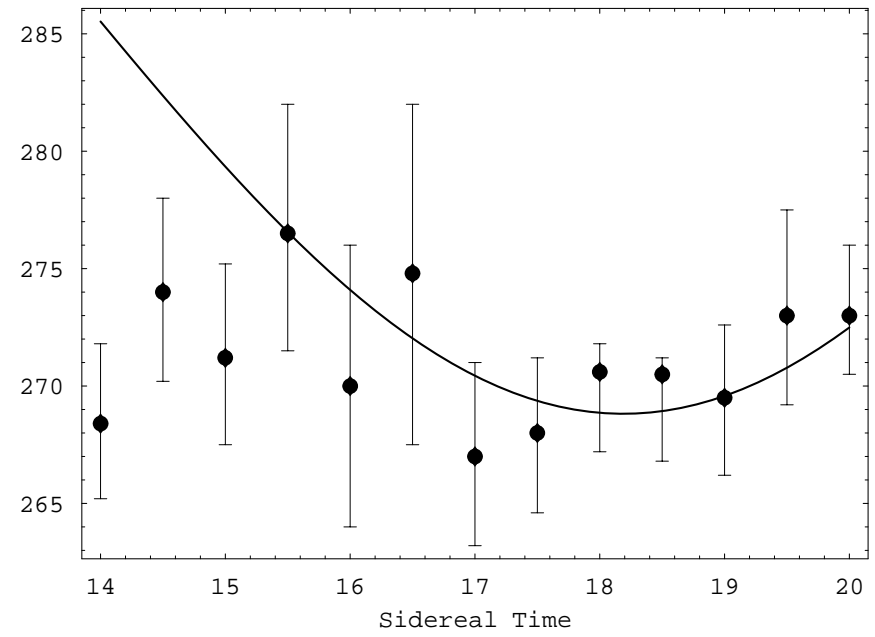

Figure 28: Frequency difference in $\mathrm{kHz}$ between the two masers in the 1963 New Bedford experiment after a $90^{\circ}$ rotation. The $275 \mathrm{kHz}$ difference is a systematic repeatable apparatus effect, whereas the superimposed 'dip' at $17-18: 00^{h r}$ sidereal time of approximately $3 \mathrm{kHz}$ is a real time dependent frequency difference. The full curve shows the theoretical prediction for the time of the 'dip' for this experiment using the Miller direction for $\hat{\mathbf{v}}\left(\alpha=5.2^{h r}, \delta=-67^{0}\right)$ with $|\mathbf{v}|=443 \mathrm{~km} / \mathrm{s}$ and including the earth's orbital velocity and sun gravitational in-flow velocity effects for January 20, 1963. The absolute scale of this theoretical prediction was not possible to compute as the refractive index of the He-Ne gas mixture was unknown.

racy by ignoring relativistic effects, which are 2 nd-order in $v / c$. Let the projection of the absolute velocity vector $\mathbf{v}$ onto the direction of the coaxial cable be $v_{P}$ as before. Then the phase comparators reveal the difference ${ }^{16}$ between the propagation times in NS and SN directions. First consider the analysis with no Fresnel drag effect, Sect.16.2,

$$
\begin{aligned}
\Delta t & =\frac{L}{\frac{c}{n}-v_{P}}-\frac{L}{\frac{c}{n}+v_{P}}, \\
& =2 \frac{L}{c / n} n \frac{v_{P}}{c}+O\left(\frac{v_{P}^{2}}{c^{2}}\right) \approx 2 t_{0} n \frac{v_{P}}{c} .
\end{aligned}
$$

Here $L=1.5 \mathrm{~km}$ is the length of the coaxial cable, $n=1.5$ is the refractive index of the insulator within the coaxial cable, so that the speed of the RF signals is approximately $c / n=200,000 \mathrm{~km} / \mathrm{s}$, and so $t_{0}=n L / c=7.5 \times 10^{-6} \mathrm{sec}$ is the one-way RF travel time when $v_{P}=0$. Then, for example, a value of $v_{P}=400 \mathrm{~km} / \mathrm{s}$ would give $\Delta t=30 \mathrm{~ns}$. Because Brussels has a latitude of $51^{0} \mathrm{~N}$ then for the Miller direction the projection

\footnotetext{
${ }^{16}$ The measurement protocol in Sect.9.2 uses the sum of the travel times.
} 


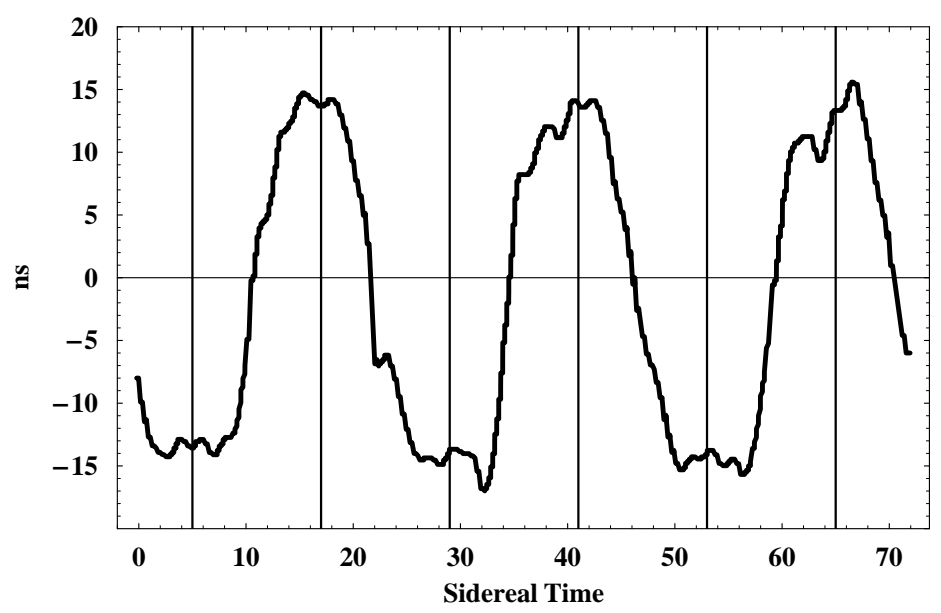

Figure 29: Variations in twice the one-way travel time, in ns, for an RF signal to travel $1.5 \mathrm{~km}$ through a coaxial cable between Rue du Marais and Rue de la Paille, Brussels. An offset has been used such that the average is zero. The definition of the sign convention for $\Delta t$ used by DeWitte is unclear. The cable has a North-South orientation, and the data is \pm difference of the travel times for NS and SN propagation. The sidereal time for maximum effect of $\sim 17 \mathrm{hr}$ (or $\sim 5 \mathrm{hr}$ ) (indicated by vertical lines) agrees with the direction found by Miller and also by Jaseja et al., but because of the ambiguity in the definition of $\Delta t$ the opposite direction would also be consistent with this data. Plot shows data over 3 sidereal days and is plotted against sidereal time. See Fig.30b for theoretical predictions for one sidereal day. The time of the year of the data is not identified. The fluctuations are evidence of turbulence associated with the gravitational in-flow towards the sun. Adapted from DeWitte [103].

effect is such that $v_{P}$ almost varies from zero to a maximum value of $|\mathbf{v}|$. The DeWitte data in Fig.29 shows $\Delta t$ plotted with a false zero, but shows a variation of some 28 ns. So the DeWitte data is in excellent agreement with the Miller's data ${ }^{17}$. The Miller experiment has thus been confirmed by a non-interferometer experiment if we ignore a Fresnel drag.

But if we include a Fresnel drag effect then the change in travel time $\Delta t_{F}$ becomes

$$
\begin{aligned}
\Delta t_{F} & =\frac{L}{\frac{c}{n}+b v_{P}-v_{P}}-\frac{L}{\frac{c}{n}-b v_{P}+v_{P}}, \\
& =2 \frac{L}{c} \frac{v_{P}}{c}+O\left(\frac{v_{P}^{2}}{c^{2}}\right)
\end{aligned}
$$

\footnotetext{
${ }^{17}$ There is ambiguity in Ref.[103] as to whether the time variations in Fig.29 include the factor of 2 or not, as defined in (98). It is assumed here that a factor of 2 is included.
} 


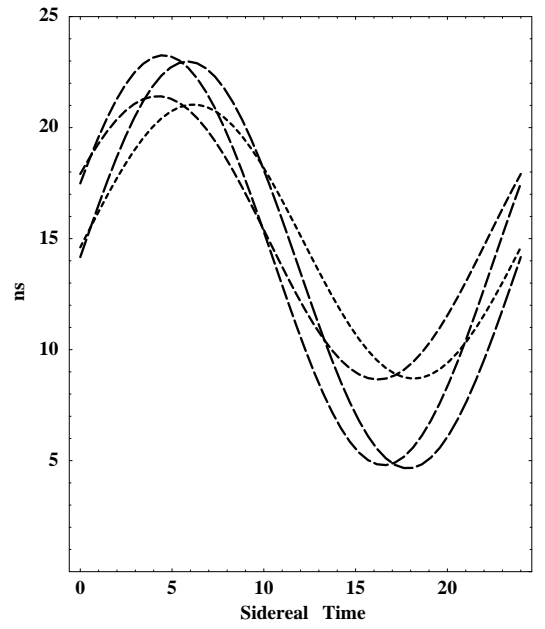

(a)

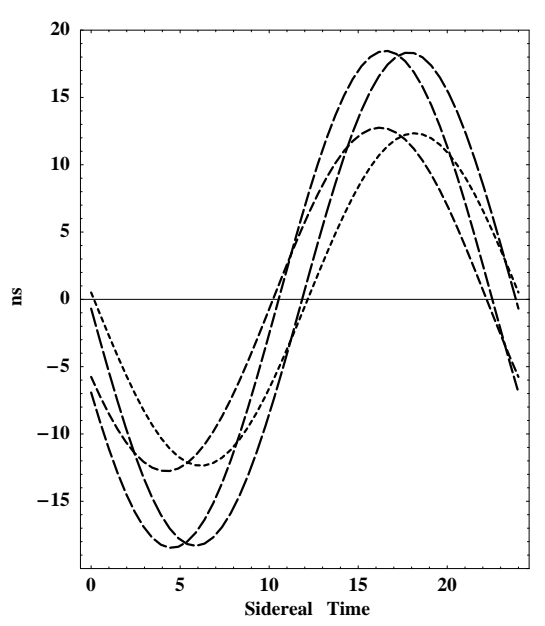

(b)

Figure 30: Theoretical predictions for the variations in travel time, in ns, for one sidereal day, in the DeWitte Brussels coaxial cable experiment for $\mathbf{v}_{\text {cosmic }}$ in the direction $(\alpha, \delta)=\left(5.2^{h},-67^{0}\right)$ and with the Miller magnitude of $443 \mathrm{~km} / \mathrm{s}$, and including orbital and in-flow effects (but without turbulence). Shown are the results for four days: for the Vernal Equinox, March 21 (shortest dashes), and for 90, 180 and 270 days later (shown with increasing dash length). Figure (a) Shows change in one-way travel time $t_{0} n v_{P} / c$ for signal travelling from $\mathrm{N}$ to $\mathrm{S}$. Figure (b) shows $\Delta t$, as defined in (98), with an offset such that the average is zero so as to enable comparison with the data in Fig.29. $\Delta t$ is twice the one-way travel time. For the direction opposite to $(\alpha, \delta)=\left(5.2^{h},-67^{0}\right)$ the same curves arise except that the identification of the months is different and the sign of $\Delta t$ also changes. The sign of $\Delta t$ determines which of the two directions is the actual direction of absolute motion. However the definition of the sign convention for $\Delta t$ used by DeWitte is unclear.

$$
=\frac{1}{n^{2}} \Delta t
$$

where $b=1-1 / n^{2}$ is the Fresnel drag coefficient, see Sect.16.2. Then $\Delta t_{F}$ is smaller than $\Delta t$ by a factor of $n^{2}=1.5^{2}=2.25$, and so a speed of $v_{P}=2.25 \times 400=900 \mathrm{~km} / \mathrm{s}$ would be required to produce a $\Delta t_{F}=30 \mathrm{~ns}$. This speed is inconsistent with the results from gas-mode interferometer experiments, and also inconsistent with the data from the Torr-Kolen gas-mode coaxial cable experiment, Sect.10.9. This raises the question as to whether the Fresnel effect is present in transparent solids, and indeed whether it has ever been studied? As well we are assuming the conventional eletromagnetic theory for the $\mathrm{RF}$ fields in the coaxial cable. An experiment to investigate this is underway at Flinders university, as discussed in Sect.12.3. 


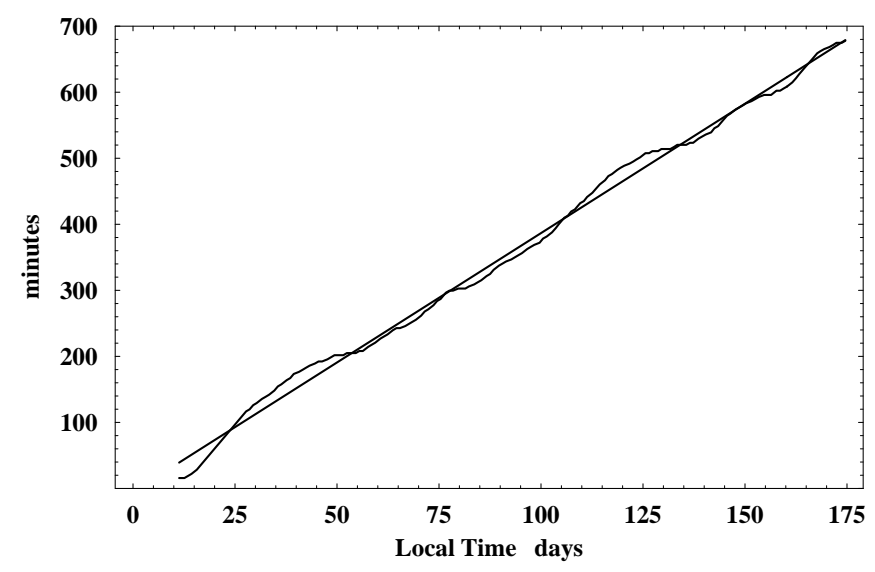

Figure 31: Plot of the negative of the drift of the cross-over time between minimum and maximum travel-time variation each day (at $\sim 10^{h} \pm 1^{h} \mathrm{ST}$ ) versus local solar time for some 180 days. The straight line plot is the least squares fit to the experimental data, giving an average slope of 3.92 minutes/day. The time difference between a sidereal day and a solar day is 3.93 minutes/day. This demonstrates that the effect is related to sidereal time and not local solar time. The actual days of the year are not identified in Ref.[103]. Adapted from DeWitte [103].

The actual days of the data in Fig.29 are not revealed in Ref.[103] so a detailed analysis of the DeWitte data is not possible. Nevertheless theoretical predictions for various days in a year are shown in Fig. 30 using the speed of $v_{\text {cosmic }}=433 \mathrm{~km} / \mathrm{s}$ and where the diurnal effects of the earth's orbital velocity and the gravitational in-flow cause the range of variation of $\Delta t$ and sidereal time of maximum effect to vary throughout the year. The predictions give $\Delta t=30 \pm 4$ ns over a year compared to the DeWitte value of 28 ns in Fig.29. If all of DeWitte's 178 days of data were available then a detailed analysis would be possible.

Ref.[103] does however reveal the sidereal time of the cross-over time, that is a 'zero' time in Fig.29, for all 178 days of data. This is plotted in Fig.31 and demonstrates that the time variations are correlated with sidereal time and not local solar time. A least squares best fit of a linear relation to that data gives that the cross-over time is retarded, on average, by 3.92 minutes per solar day. This is to be compared with the fact that a sidereal day is 3.93 minutes shorter than a solar day. So the effect is certainly cosmological and not associated with any daily thermal effects, which in any case would be very small as the cable is buried. Miller had also compared his data against sidereal time and established the same property, namely that up to small diurnal effects identifiable with the arth's orbital motion, features in the data tracked sidereal time and not solar time; see Ref.[89] for a detailed analysis. 
The DeWitte data is also capable of resolving the question of the absolute direction of motion found by Miller. Is the direction $(\alpha, \delta)=\left(5.2^{h},-67^{0}\right)$ or the approximately opposite direction? By doing a 2nd-order Michelson interferometer experiment Miller had to rely on the earth's diurnal effects in order to resolve this ambiguity, but his analysis of course did not take account of the gravitational in-flow effect, and so until a re-analysis of his data his preferred choice of direction must remain to be confirmed. The DeWitte experiment could easily resolve this ambiguity by simply noting the sign of $\Delta t$. Unfortunately it is unclear in Ref.[103] as to how the sign in Fig.29 is actually defined, and DeWitte does not report a direction expecting, as he did, that the direction should have been the same as the CMB direction.

The DeWitte observations were truly remarkable considering that initially they were serendipitous. They demonstrated yet again that the Einstein postulates were in contradiction with experiment. To my knowledge no physics journal ${ }^{18}$ has published a report of the DeWitte experiment and DeWitte himself reports [103] that he was dismissed from Belgacom. I have been unsuccessful in contacting Roland DeWitte. His data like those of Miller are extremely valuable and need to be made available for detailed analysis.

That the DeWitte experiment is not a gas-mode Michelson interferometer experiment is very significant. The value of the speed of absolute motion revealed by the DeWitte experiment of some $400 \mathrm{~km} / \mathrm{s}$ is in agreement with the speeds revealed by the new analysis of various Michelson interferometer data, which use the recently discovered refractive index effect, see Fig.24. Not only was this effect confirmed by comparing results for different gases, but the re-scaling of the older $v_{M}$ speeds to $v=v_{M} / \sqrt{n^{2}-1}$ speeds resulting from this effect are now confirmed. A new and much simpler 1st-order experiment is discussed in Sect.12.3 which avoids the use of atomic clocks.

\subsection{The Torr-Kolen Experiment: 1981}

A coaxial cable experiment similar to but before the DeWitte experiment was performed at the University of Utah in 1981 by Torr and Kolen [104]. This involved two rubidium vapor clocks placed approximately $500 \mathrm{~m}$ apart with a $5 \mathrm{MHz}$ sinewave $\mathrm{RF}$ signal propagating between the clocks via a nitrogen filled coaxial cable maintained at a constant pressure of $\sim 2$ psi. This means that the Fresnel drag effect is not important in this experiment. Unfortunately the cable was orientated in an East-West direction which is not a favourable orientation for observing absolute motion in the Miller direction, unlike the Brussels North-South cable orientation. There is no reference to Miller's result in the

\footnotetext{
${ }^{18}$ Papers reporting or analysing absolute motion and related effects are banned from physics journals. This appears to be based on the almost universal misunderstanding by physicists that absolute motion is incompatible with the many confirmed relativistic effects.
} 


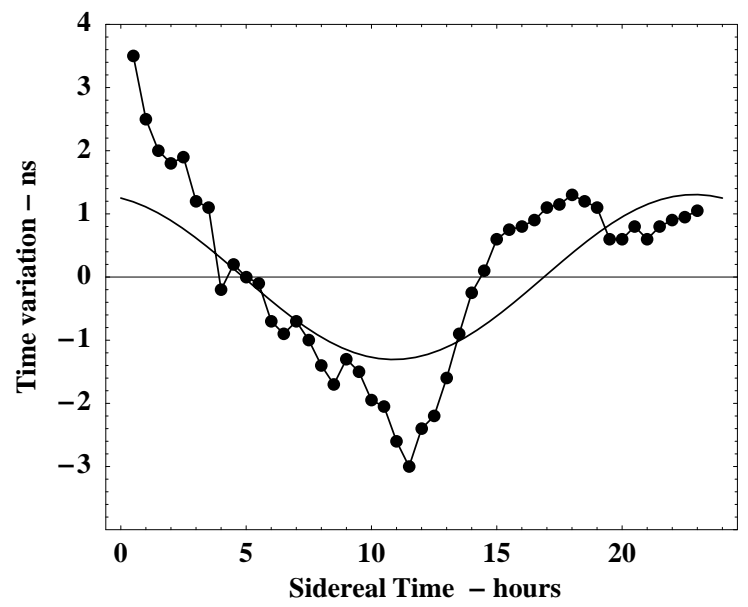

Figure 32: Data from the 1981 Torr-Kolen experiment at Logan, Utah [104]. The data shows variations in travel times (ns), for local times, of an RF signal travelling through $500 \mathrm{~m}$ of coaxial cable orientated in an E-W direction. Actual days are not indicated but the experiment was done during February-June 1981. Results are for a typical day. For the 1st of February the local time of 12:00 corresponds to 13:00 sidereal time. The predictions are for February, for a cosmic speed of $430 \mathrm{~km} / \mathrm{s}$ in the direction $(\alpha, \delta)=\left(5.2^{h},-67^{0}\right)$, and including orbital and in-flow velocities but without theoretical turbulence.

Torr and Kolen paper, otherwise they would presumably not have used this orientation. Nevertheless there is a projection of the absolute motion velocity onto the East-West cable and Torr and Kolen did observe an effect in that, while the round speed time remained constant within $0.0001 \%$ c, typical variations in the one-way travel time were observed, as shown in Fig.32 by the data points. The theoretical predictions for the TorrKolen experiment for a cosmic speed of $433 \mathrm{~km} / \mathrm{s}$ in the direction $(\alpha, \delta)=\left(5.2^{h},-67^{0}\right)$, and including orbital and in-flow velocities, are shown in Fig.32. As well the maximum effect occurred, typically, at the predicted times. So the results of this experiment are also in remarkable agreement with the Miller direction, and the speed of $433 \mathrm{~km} / \mathrm{s}$ which of course only arises after re-scaling the Miller speeds for the effects of the gravitational in-flow. As well Torr and Kolen reported fluctuations in both the magnitude, from 1 - 3 $\mathrm{ns}$, and time of the maximum variations in travel time just as DeWitte observed some 10 years later. Again we argue that these fluctuations are evidence of genuine turbulence in the in-flow as discussed in Sect.11.3. So the Torr-Kolen experiment again shows strong evidence for the new theory of gravity, and which is over and above its confirmation of the various observations of absolute motion. 


\section{Gravitational In-Flow and Gravitational Waves}

\subsection{Gravitational In-flow from the Miller Data}

As already noted Miller was led to the conclusion that for reasons unknown the existing theory of the Michelson interferometer did not reveal true values of $v_{P}$, and for this reason he introduced the parameter $k$, with $\bar{k}$ indicating his numerical values. Miller had reasoned that he could determine both $\mathbf{v}_{\text {cosmic }}$ and $\bar{k}$ by observing the interferometer determined $v_{P}$ and $\psi$ over a year because the known orbital velocity of the earth about the sun would modulate both of these observables, and by a scaling argument he could determine the absolute velocity of the solar system. In this manner he finally determined that $\left|\mathbf{v}_{\text {cosmic }}\right|=208 \mathrm{~km} / \mathrm{s}$ in the direction $\left(\alpha=4^{h r} 54^{m}, \delta=-70^{0} 33^{\prime}\right)$. However now that the theory of the Michelson interferometer has been revealed an anomaly becomes apparent. Table 3 shows $v=v_{M} / k_{\text {air }}$ for each of the four epochs, giving speeds consistent with the revised Michelson-Morley data. However Table 3 also shows that $\bar{k}$ and the speeds $\bar{v}=v_{M} / \bar{k}$ determined by the scaling argument are considerably different. Here the $v_{M}$ values arise after taking account of the projection effect. That $\bar{k}$ is considerably larger than the value of $k_{a i r}$ indicates that another velocity component has been overlooked. Miller of course only knew of the tangential orbital speed of the earth, whereas the new physics predicts that as-well there is a quantum-gravity radial in-flow $\mathbf{v}_{\text {in }}$ of the quantum foam. We can re-analyse Miller's data to extract a first approximation to the speed of this in-flow component. Clearly it is $v_{R}=\sqrt{v_{\text {in }}^{2}+v_{\text {tangent }}^{2}}$ that sets the scale and not $v_{\text {tangent }}$, and because $\bar{k}=v_{M} / v_{\text {tangent }}$ and $k_{\text {air }}=v_{M} / v_{R}$ are the scaling relations, then

$$
\begin{aligned}
v_{\text {in }} & =v_{\text {tangent }} \sqrt{\frac{v_{R}^{2}}{v_{\text {tangent }}^{2}}-1}, \\
& =v_{\text {tangent }} \sqrt{\frac{\bar{k}^{2}}{k_{\text {air }}^{2}}-1 .}
\end{aligned}
$$

Using the $\bar{k}$ values in Table 3 and the value ${ }^{19}$ of $k_{\text {air }}$ we obtain the $v_{\text {in }}$ speeds shown in Table 3, which give an average speed of $54 \mathrm{~km} / \mathrm{s}$, compared to the 'Newtonian' in-flow speed of $42 \mathrm{~km} / \mathrm{s}$. Note that the in-flow interpretation of the anomaly predicts that $\bar{k}=\left(v_{R} / v_{\text {tangent }}\right) k_{\text {air }}=\sqrt{3} k_{\text {air }}=0.042$. Alternatively one can simply rescale $\bar{v}$ by $\sqrt{3}$

\footnotetext{
${ }^{19}$ We have not modified this value to take account of the altitude effect or temperatures atop Mt.Wilson. This weather information was not recorded by Miller. The temperature and pressure effect is that $n=1.0+0.00029 \frac{P}{P_{0}} \frac{T_{0}}{T}$, where $T$ is the temperature in ${ }^{0} \mathrm{~K}$ and $P$ is the pressure in atmospheres. $T_{0}=273 \mathrm{~K}$ and $P_{0}=1 \mathrm{~atm}$.
} 
because $v_{R}=\sqrt{3} v_{\text {in }}$ instead of $v_{i n}$ sets the scale. Of course this simple re-scaling of the Miller results is not completely valid because (i) the direction of $\mathbf{v}_{R}$ is of course different to that of $\mathbf{v}_{\text {tangent }}$, and also not necessarily orthogonal to $\mathbf{v}_{\text {tangent }}$ because of turbulence, and (ii) also because of turbulence we would expect some contribution from the in-flow effect of the earth itself, namely that it is not always perpendicular to the earth's surface, and so would give a contribution to a horizontally operated interferometer.

\begin{tabular}{|l|l|c|l|l|l|l|}
\hline Epoch & \multicolumn{1}{|c|}{$v_{M}$} & $\bar{k}$ & $v=v_{M} / k_{a i r}$ & $\bar{v}=v_{M} / \bar{k}$ & $v=\sqrt{3} \bar{v}$ & $v_{\text {in }}$ \\
\hline \hline February 8 & $9.3 \mathrm{~km} / \mathrm{s}$ & 0.048 & $385.9 \mathrm{~km} / \mathrm{s}$ & $193.8 \mathrm{~km} / \mathrm{s}$ & $335.7 \mathrm{~km} / \mathrm{s}$ & $51.7 \mathrm{~km} / \mathrm{s}$ \\
\hline April 1 & 10.1 & 0.051 & 419.1 & 198.0 & 342.9 & 56.0 \\
\hline August 1 & 11.2 & 0.053 & 464.7 & 211.3 & 366.0 & 58.8 \\
\hline September 15 & 9.6 & 0.046 & 398.3 & 208.7 & 361.5 & 48.8 \\
\hline \hline
\end{tabular}

Table 3. The $\bar{k}$ anomaly, $\bar{k} \gg k_{\text {air }}=0.0241$, as the gravitational in-flow effect. Here $v_{M}$ and $\bar{k}$ come from fitting the interferometer data (using $v_{\text {in }}$ to determine $\bar{k}$ ), while $v$ and $\bar{v}$ are computed speeds using the indicated scaling. The average of the in-flow speeds is $v_{\text {in }}=54 \pm 5 \mathrm{~km} / \mathrm{s}$, compared to the 'Newtonian' in-flow speed of $42 \mathrm{~km} / \mathrm{s}$. From column 4 we obtain the average $v=417 \pm 40 \mathrm{~km} / \mathrm{s}$.

An analysis that properly searches for the in-flow velocity effect clearly requires a complete re-analysis of the Miller data, and this is now possible and underway at Flinders University as the original data sheets have been found. It should be noted that the direction almost diametrically opposite $\left(\alpha=4^{h r} 54^{m}, \delta=-70^{0} 33^{\prime}\right)$, namely ( $\alpha=17^{h r}, \delta=+68^{\prime}$ ) was at one stage considered by Miller as being possible. This is because the Michelson interferometer, being a 2nd-order device, has a directional ambiguity which can only be resolved by using the diurnal motion of the earth. However as Miller did not include the in-flow velocity effect in his analysis it is possible that a re-analysis might give this northerly direction as the direction of absolute motion of the solar system.

Fig.33 shows the so called 'aberration orbit' which indicates the change in direction of absolute motion at various epochs due to the vector sum of the three main contributions $\mathbf{v}_{\text {cosmic }}+\mathbf{v}_{\text {tangent }}-\mathbf{v}_{\text {in }}$, with the latter two components having a diurnal time-dependence. Note that Miller's data points in an analogous plot, Fig.28 in Ref.[89], involved for February the average of 8080 single interferometer observations, and for the others 7680 for August, 6640 for September and 3208 for April. So Miller's observations were very thorough.

Hence not only did Miller observe absolute motion, as he claimed, but the quality and quantity of his data has also enabled the confirmation of the existence of the gravitational in-flow effect $[13,14]$. This is a manifestation of a new theory of gravity and one which relates to quantum gravitational effects via the unification of matter and space developed 


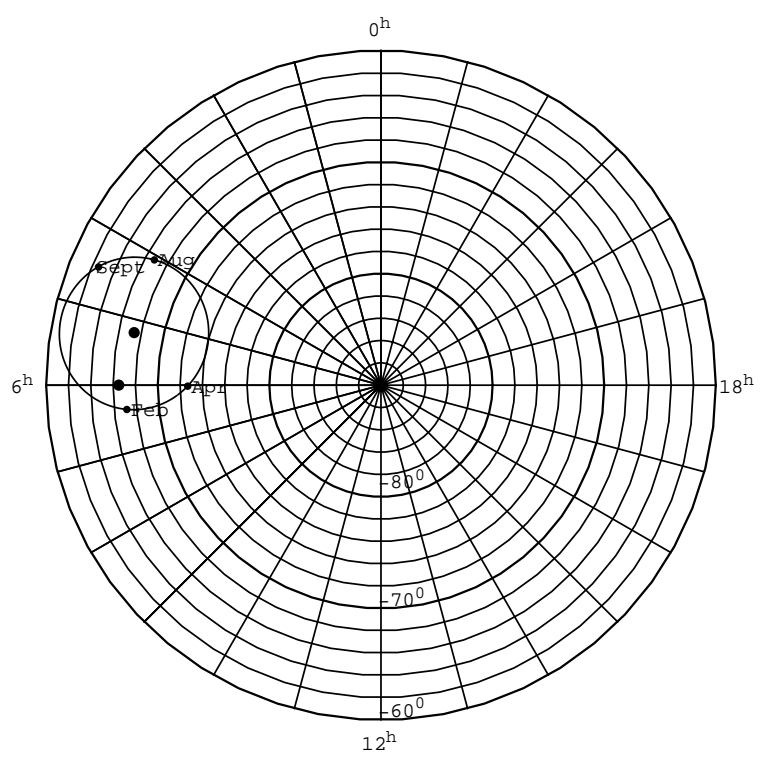

Figure 33: The 'Aberration Orbit' shows the net direction of absolute motion at various epochs. The plot shows the south circumpolar region of the celestial sphere. The direction $\left(\alpha=6^{h r}, \delta=-66.5^{0}\right)$ is the pole of the ecliptic. At the centre of the aberration orbit is the direction $\left(\alpha=5.2^{h r}, \delta=-67^{0}\right)$. Compared to Miller's analogous plot, Fig.28 in Ref.[89], the inclusion of the in-flow effect has caused the epoch points to be rotated around the aberration orbit.

in previous sections. As well the persistent evidence that this in-flow is turbulent indicates that this theory of gravity involves self-interaction of space itself.

\subsection{Galactic In-flow and the CMB Frame}

Absolute motion (AM) of the solar system has been observed in the direction $(\alpha=$ $\left.5.2^{h}, \delta=-67^{0}\right)$, up to an overall sign to be sorted out, with a speed of $417 \pm 40 \mathrm{~km} / \mathrm{s}$. This is the velocity after removing the contribution of the earth's orbital speed and the sun in-flow effect. It is significant that this velocity is different to that associated with the Cosmic Microwave Background ${ }^{20}$ (CMB) relative to which the solar system has a speed

\footnotetext{
${ }^{20}$ The understanding of the galactic in-flow effect was not immediate: In [12] the direction was not determined, though the speed was found to be comparable to the CMB determined speed. In [13] the fact that the directions were very different was noted but not appreciated, and in fact thought to be due to experimental error. In [14] an analysis of some of the 'smoother' MichelsonMorley data resulted in an incorrect direction. At that stage it was not understood that the data showed large fluctuations in the azimuth apparently caused by the turbulence. Here the issue is hopefully finally resolved.
} 
of $369 \mathrm{~km} / \mathrm{s}$ in the direction $\left(\alpha=11.20^{h}, \delta=-7.22^{0}\right)$, see [101]. This CMB velocity is obtained by finding the preferred frame in which this thermalised $3^{0} \mathrm{~K}$ radiation is isotropic, that is by removing the dipole component. The CMB velocity is a measure of the motion of the solar system relative to the universe as a whole, or aleast a shell of the universe some 15Gyrs away, and indeed the near uniformity of that radiation in all directions demonstrates that we may meaningfully refer to the spatial structure of the universe. The concept here is that at the time of decoupling of this radiation from matter that matter was on the whole, apart from small observable fluctuations, at rest with respect to the quantum-foam system that is space. So the CMB velocity is the motion of the solar system with respect to space universally, but not necessarily with respect to the local space. Contributions to this velocity would arise from the orbital motion of the solar system within the Milky Way galaxy, which has a speed of some 250 $\mathrm{km} / \mathrm{s}$, and contributions from the motion of the Milky Way within the local cluster, and so on to perhaps larger clusters.

On the other hand the AM velocity is a vector sum of this universal CMB velocity and the net velocity associated with the local gravitational in-flows into the Milky Way and the local cluster. If the CMB velocity had been identical to the AM velocity then the in-flow interpretation of gravity would have been proven wrong. We therefore have three pieces of experimental evidence for this interpretation (i) the refractive index anomaly discussed previously in connection with the Miller data, (ii) the turbulence seen in all detections of absolute motion, and now (iii) that the AM velocity is different in both magnitude and direction from that of the CMB velocity, and that this velocity does not display the turbulence seen in the AM velocity.

That the AM and CMB velocities are different amounts to the discovery of the resolution to the 'dark matter' conjecture. Rather than the galactic velocity anomalies being caused by such undiscovered 'dark matter' we see that the in-flow into non spherical galaxies, such as the spiral Milky Way, will be non Newtonian, that is (34) is not appropriate to such highly non-spherical systems. When solutions to (75) are determined for such systems it will be possible to map out the predicted AM velocity and compare with the observed velocity. As well it will be interesting to determine, at least theoretically, the scale of turbulence expected in galactic systems, particularly as the magnitude of the turbulence seen in the AM velocity is somewhat larger than might be expected from the sun in-flow alone. Any theory for the turbulence effect will certainly be checkable within the solar system as the time scale of this is suitable for detailed observation.

It is also clear that the time of obervers at rest with respect to the CMB frame is absolute or universal time. This interpretation of the CMB frame has of course always been rejected by supporters of the SR/GR formalism. As for space we note that it has a differential structure, in that different regions are in relative motion. This is caused by 
the gravitational in-flow effect locally, and as well by the growth of the universe.

\subsection{In-Flow Turbulence and Gravitational Waves}

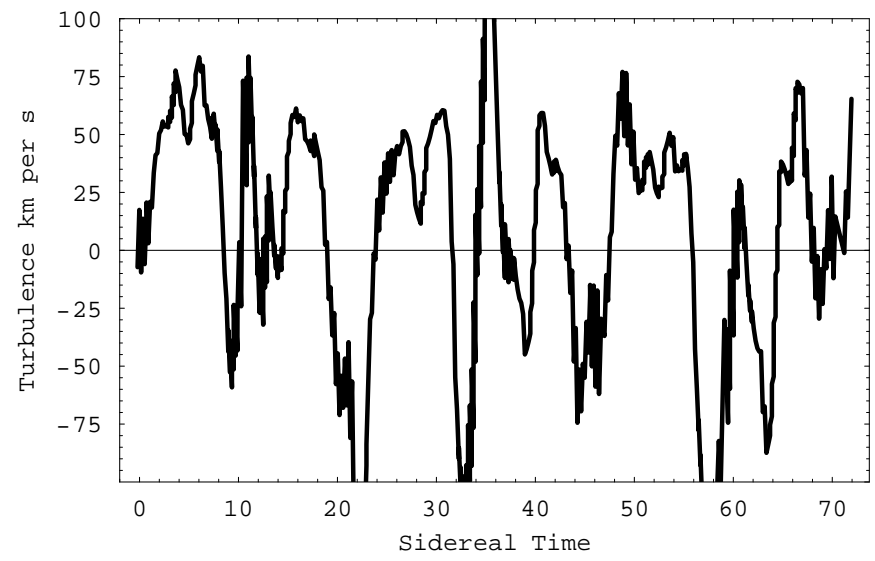

Figure 34: Speed fluctuations determined from Fig.29 by subtracting a least squares best fit of the forms shown in Fig.30b. A 1ns variation in travel time corresponds approximately to a speed variation of $27 \mathrm{~km} / \mathrm{s}$. The larger speed fluctuations actually arise from a fluctuation in the cross-over time, that is, a fluctuation in the direction of the velocity. This plot implies that the velocity flow-field is turbulent. The scale of this turbulence is comparable to that evident in the Miller data, as shown in Fig.19 and Fig.35(a).

The velocity flow-field equation, suggested to have the form (75), is expected to have solutions possessing turbulence, that is, fluctuations in both the magnitude and direction of the gravitational in-flow component of the velocity flow-field. Indeed all the Michelson interferometer experiments showed evidence of such turbulence. The first clear evidence was from the Miller experiment, as shown in Fig.19 and Fig.35(a). Miller offered no explanation for these fluctuations but in his analysis of that data he did running time averages, as shown by the smoother curves in Fig.19. Miller may have in fact have simply interpreted these fluctuations as purely instrumental effects. While some of these fluctuations may be partially caused by weather related temperature and pressure variations, the bulk of the fluctuations appear to be larger than expected from that cause alone. Even the original Michelson-Morley data in Fig.15 shows variations in the velocity field and supports this interpretation. However it is significant that the noninterferometer DeWitte data also shows evidence of turbulence in both the magnitude and direction of the velocity flow field, as shown in Fig.34. Just as the DeWitte data agrees with the Miller data for speeds and directions the magnitude fluctuations, shown 


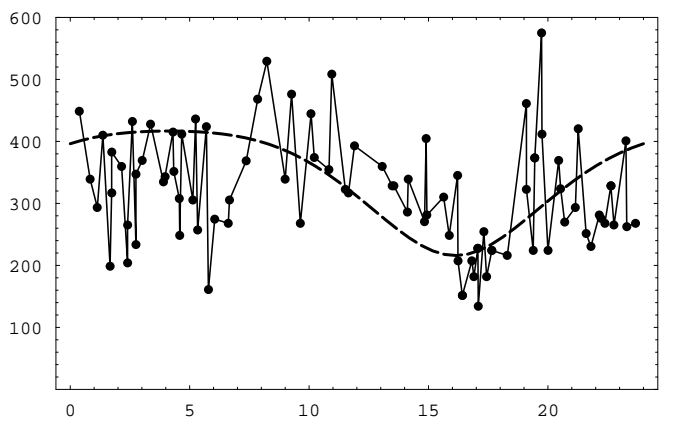

(a)

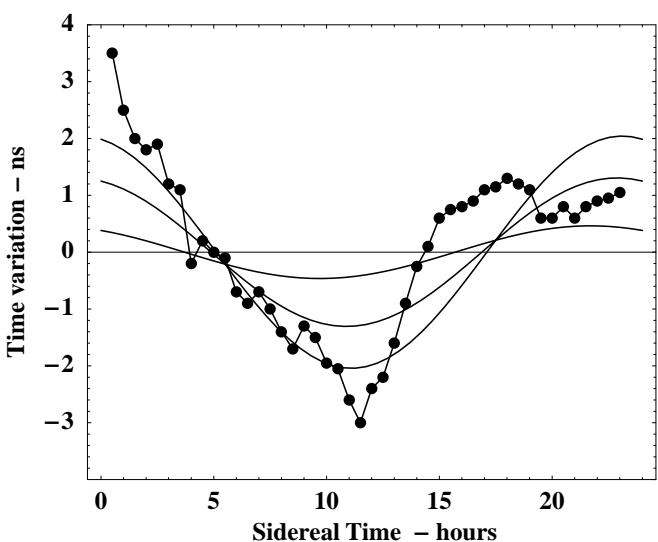

(b)

Figure 35: (a) The absolute projected speeds $v_{P}$ in the Miller experiment plotted against sidereal time in hours for September 1925, showing the variations in speed caused by the gravitational wave turbulence. and (b) similar variations in travel times when the declination is varied by $\pm 10^{0}$ about the direction $\alpha=5.2^{h}, \delta=-67^{0}$, for a cosmic speed of $433 \mathrm{~km} / \mathrm{s}$ in the Torr-Kolen experiment.

in Fig.34, are very similar in absolute magnitude to, for example, the speed turbulence shown in Fig.35a.

As well the Torr-Kolen coaxial cable experiment showed evidence of turbulent flow. Fortuitously the cable was orientated almost at $90^{\circ}$ to the direction of absolute motion, as noted in Sect.10.9. This meant that any fluctuation in the direction of the flow would have a significant effect on the magnitude of the propagation times. This is illustrated in Fig.35b for the case where the declination is varied $\pm 10^{0}$. Torr and Kollen reported fluctuations of that magnitude.

It therefore becomes clear that there is strong evidence for these fluctuations being evidence of physical turbulence in the flow field. The magnitude of this turbulence appears to be somewhat larger than that which would be caused by the in-flow of quantum foam towards the sun, and indeed following on from Sect.11.2 some of this turbulence may be associated with galactic in-flow into the Milky Way. This in-flow turbulence is a form of gravitational wave and the ability of gas-mode Michelson interferometers to detect absolute motion means that experimental evidence of such a wave phenomena has been available for a considerable period of time, but suppressed along with the detection of absolute motion itself. Of course flow equations of the form in (75) do not exhibit those gravitational waves of the form that have been predicted to exist based on the Einstein equations, and which are supposed to propagate at the speed of light. All this means that gravitational wave phenomena is very easy to detect and amounts to new physics 
that can be studied in much detail, particularly using the new 1st-order interferometer discussed in Sect.12.3.

\subsection{Gravitational Anomalies}

In Sect.9.9 anomalies associated with the measurement of $G$ were briefly discussed and it was pointed out that these were probably explainable within the new in-flow theory of gravity. There are in fact additional gravitational anomalies that are not well-known in physics, presumably because their existence is incompatible with the Newtonian or the Hilbert-Einstein gravity theories.

The most significant of these anomalies is the Allais effect [116]. In June 1954 Allais $^{21}$ reported that a Foucault pendulum exhibited peculiar movements at the time of a solar eclipse. Allais was recording the precession of a Foucault pendulum in Paris. Coincidently during the 30 day observation period a partial solar eclipse occurred at Paris on June 30. During the eclipse the precession of the pendulum was seen to be disturbed. Similar results were obtained during another solar eclipse on October 29 1959. There have been other repeats of the Allais experiment with varying results.

Another anomaly was reported by Saxl and Allen [117] during the solar eclipse of March 7 1970. Significant variations in the period of a torsional pendulum were observed both during the eclipse and as well in the hours just preceding and just following the eclipse. The effects seem to suggest that an "apparent wavelike structure has been observed over the course of many years at our Harvard laboratory", where the wavelike structure is present and reproducible even in the absence of an eclipse.

Again Zhou et al. $[118,119,120]$ report various time anomalies occurring during the solar eclipses observed using atomic clocks.

Other anomalies include the observed solar neutrino flux rate, which is one half that predicted value using the current theory of stellar structure and which is based upon the Newtonian theory of gravity for the interior of the sun. Another anomaly is the larger than predicted de-acceleration, $a_{p}=(8.74 \pm 1.33) \times 10^{-8} \mathrm{~cm} / \mathrm{s}^{2}$ directed towards the sun, of the Pioneer 10 amd 11 planetary probes as they leave the solar system [121]. This is clearly an effect expected using the new theory of gravity as the $C(\mathbf{v})$ term predicts a 'dark matter' effect even within the solar system, caused by the planets breaking the spherical symmetry of the matter distribution. Finally the mine/borehole $g$ anomaly is also an excellent example of the breakdown of Newtonian gravity, as discussed in Sect.11.5.

All these anomalies suggest that gravity has aspects to it that are not within the prevailing theories, but that the in-flow theory discussed above might well provide an

\footnotetext{
${ }^{21}$ Maurice Allais won the Noble Prize for Economics in 1988.
} 
explanation, and indeed these anomalies may well provide further phenomena that could be used to test the new theory. The effects associated with the solar eclipses could presumably follow from the alignment of the sun, moon and the earth causing enhanced turbulence. The Saxl and Allen experiment of course suggests, like the other experiments analysed above, that the turbulence is always present. To explore these anomalies detailed numerical studies of (75) are required with particular emphasis on the effect on the position of the moon.

\subsection{The Borehole g Anomaly}

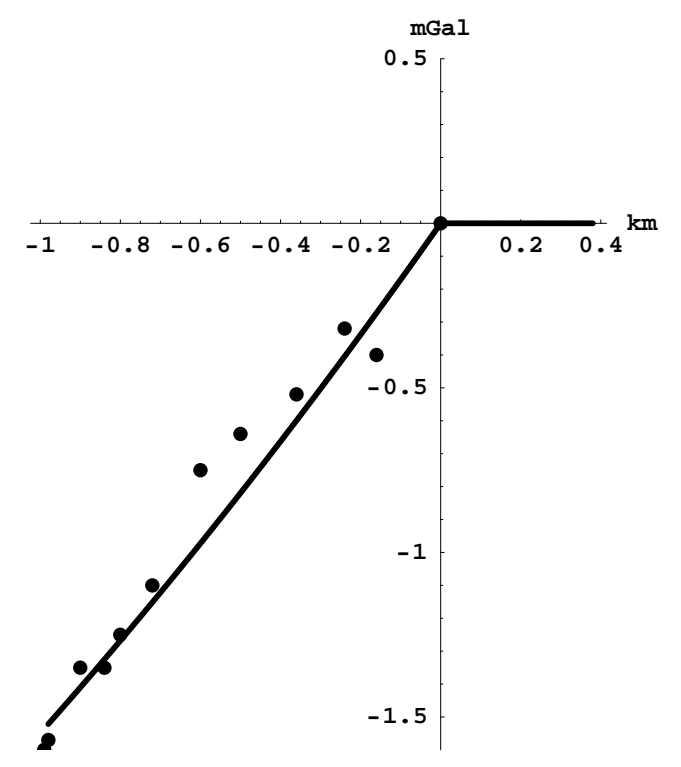

Figure 36: The data shows the gravity residuals for the Hilton mine profile, from Ref.[125], defined as $\Delta g(r)=g_{\text {Newton }}-g_{\text {observed }}$, and measured in mGal $\left(1 \mathrm{mGal}=10^{-3}\right.$ $\left.\mathrm{cm} / \mathrm{s}^{2}\right)$ plotted against depth in $\mathrm{km}$. The theory curve shows $\Delta g(r)=g_{\text {Newton }}-g_{\text {InFlow }}$ from solving (101) and (102) for a density $\rho=2760 \mathrm{~kg} / \mathrm{m}^{3}$ appropriate to the Hilton mine, a coefficient $\delta=1$ and $G=0.99925 G_{N}$.

Stacey and others $[122,123,125]$ have found evidence for non-Newtonian gravitation from gravimetric measurements (Airy experiments) in mines and boreholes. The discovery was that the measured value of $g$ down mines and boreholes became greater than that predicted by the Newtonian theory, given the density profile $\rho(r)$ implied by sampling, and so implying a defect in Newtonian gravity, as shown in Fig.36 for the Hilton mine. The results were interpreted and analysed using either a value of $G$ different to but larger than that found in laboratory experiments or by assuming a short range Yukawa type 
force in addition to the Newtonian 'inverse-square law'. Numerous experiments were carried out in which $g$ was measured as a function of depth, and also as a function of height above ground level using towers. The tower experiments $[126,127]$ did not indicate any non-Newtonian effect, and so implied that the extra Yukawa force explanation was not viable. The combined results appeared to have resulted in confusion and eventually the experimental effect was dismissed as being caused by erroneous density sampling. However the new theory of gravity predicts such an effect, and in particular that the effect should manifest within the earth but not above it, as was in fact observed. That is $\frac{d \Delta g(r)}{d r}$ should be discontinuous at the boundary, as shown in Fig.36. Essentially this effect is caused by the new $C(\mathbf{v})$ term in the in-flow theory of gravity which, as we have noted earlier, is active whenever there is a lack of complete spherical symmetry, or even within matter when there is spherical symmetry.

The Newtonian in-flow equation (33) for the velocity field becomes for systems with spherical symmetry

$$
2 \frac{v v^{\prime}}{r}+\left(v^{\prime}\right)^{2}+v v^{\prime \prime}=-4 \pi \rho(r) G_{N},
$$

where $v=v(r)$ and $v^{\prime}=\frac{d v(r)}{d r}$. The value of $v$ at the earth's surface is approximately $11 \mathrm{~km} / \mathrm{s}$. This formulation is completely equivalent to the conventional formulation of Newtonian gravity,

In the new gravity theory the in-flow equation (75) has the additional $C(\mathbf{v})$ term which, in the case of time-independent flows and spherical symmetry, becomes the term in the brackets in (102) with coefficient $\delta$,

$$
2 \frac{v v^{\prime}}{r}+\left(v^{\prime}\right)^{2}+v v^{\prime \prime}+\delta\left(\frac{v^{2}}{2 r^{2}}+\frac{v v^{\prime}}{r}\right)=-4 \pi \rho(r) G .
$$

It is important to note that the value of $G$ is not necessarily the same as the conventional value denoted as $G_{N}$. Both of these equations may be integrated in from the surface, assuming that the in-flow velocity field at or above the surface is given by

$$
v(r)=\sqrt{\frac{2 G_{N} M}{r}},
$$

so that it corresponds to the observed surface value of $g$. In (101) $M$ is the total matter content of the earth, but in (102) $M$ is the sum of the matter content and the effective total 'dark matter' content of the earth. Then above the surface, where $\rho=0$, both flow equations have (103) as identical solutions, since for this velocity field the additional bracketed term in (102) is identically zero. This explains why the tower experiments found no non-Newtonian effects. The in-flow equations may be numerically integrated inward from the surface using as boundary conditions the continuity of $v(r)$ and $v^{\prime}(r)$ at the surface. For each the $g(r)$ is determined. Fig.36 shows the resulting difference 
$\Delta g(r)=g_{\text {Newton }}-g_{\text {InFlow }}$ compared with the measured anomaly $\Delta g(r)=g_{\text {Newton }}-$ $g_{\text {observed }}$. Assuming $\delta=1$ the value of $G$ was adjusted to agree with the data, giving $G=0.99925 G_{N}$, as shown in Fig.36 (various combinations of values for $\delta$ and $G$ are equally good). However it should be noted that the data in Fig.36 was adjusted for density irregularities using Newtonian gravity, and this is now seen to be an invalid procedure. Nevertheless the results imply that a repeat of the borehole measurements would be very useful in contributing to the testing of the new theory of gravity, or perhaps even a re-analysis of existing data. The key signature of the effect, as shown in Fig.36, is the discontinuity in the slope of $\Delta g$ at the surface, and which is a consequence of the special property of the $C(\mathbf{v})$ term. Of course using a Yukawa force added to Newtonian gravity cannot produce this key signature, as such a force results in the slope of $\Delta g$ being continuous at the surface.

\subsection{Absolute Motion and Quantum Gravity}

Absolute rotational motion had been recognised as a meaningful and obervable phenomenon from the very beginning of physics. Newton had used his rotating bucket experiment to illustrate the reality of absolute rotational motion, and later Foucault and Sagnac provided further experimental proof. But for absolute linear motion the history would turm out to be completely different. It was generally thought that absolute linear motion was undetectable, at least until Maxwell's electromagnetic theory appeared to require it. In perhaps the most bizarre sequence of events in modern science it turns out that absolute linear motion has been apparent within experimental data for over 100 years. It was missed in the first experiment designed to detect it and from then on for a variety of sociological reasons it became a concept rejected by physicists and banned from their journals despite continuing new experimental evidence. Those who pursued the scientific evidence were treated with scorn and ridicule. Even worse was the impasse that this obstruction of the scientific process resulted in, namely the halting of nearly all progress in furthering our understanding of the phenomena of gravity. For it is clear from all the experiments that were capable of detecting absolute motion that there is present in that data evidence of turbulence within the velocity field. Both the in-flow itself and the turbulence are manifestations at a classical level of what is essentially quantum gravity processes.

Process Physics has given a unification of explanation and description of physical phenomena based upon the limitations of formal syntactical systems which had nevertheless achieved a remarkable encapsulation of many phenomena, albeit in a disjointed and confused manner, and with a dysfunctional ontology attached for good measure. As argued in early sections space is a quantum system continually classicalised by on-going 
non-local collapse processes. The emergent phenomenon is foundational to existence and experientialism. Gravity in this system is caused by differences in the rate of processing of the cellular information within the network which we experience as space, and consequentially there is a differential flow of information which can be affected by the presence of matter or even by space itself. Of course the motion of matter including photons with respect to that spatial information content is detectable because it affects the geometrical and chronological attributes of that matter, and the experimental evidence for this has been exhaustively discussed in this section. What has become very clear is that the phenomenon of gravity is only understandable once we have this unification of the quantum phenomena of matter and the quantum phenomena of space itself. In Process Physics the difference between matter and space is subtle. It comes down to the difference between informational patterns that are topologically preserved and those information patterns that are not. One outcome of this unification is that as a consequence of having a quantum phenomenon of space itself we obtain an informational explanation for gravity, and which at a suitable level has an emergent quantum description. In this sense we have an emergent quantum theory of gravity. Of course no such quantum description of gravity is derivable from quantising Einsteinian gravity itself. This follows on two counts, one is that the Einstein gravity formalism fails on several levels, as discussed previously, and second that quantisation has no validity as a means of uncovering deeper physics. Most surprising of all is that having uncovered the logical necessity for gravitational phenomena it also appears that even the seemingly well-founded Newtonian account of gravity has major failings. The denial of this possibility has resulted in an unproductive search for dark matter. Indeed like dark matter and spacetime much of present day physics has all the hallmarks of another episode of Ptolemy's epicycles, namely concepts that appear to be well founded but in the end turn out to be illusions, and ones that have acquired the status of dogma.

If the Michelson-Morley experiment had been properly analysed and the phenomena revealed by the data exposed, and this would have required in 1887 that Newtonian physics be altered, then as well as the subsequent path of physics being very different, physicists would almost certainly have discovered both the gravitational in-flow effect and associated turbulence. It is not beyond expectation that a modification of Newtonian gravity in terms of a velocity field and the extra term in (75) would have been quickly developed. Even Newton had speculated about an in-flow explanation for gravity.

It is clear then that observation and measurement of absolute motion leads directly to a changed paradigm regarding the nature and manifestations of gravitational phenomena, and that the new 1st-order interferometer described in Sect.12.3 will provide an extremely simple device to uncover aspects of gravity previously denied by current physics. There are two aspects of such an experimental program. One is the characterisation of the 
turbulence and its linking to the new non-linear term in the velocity field theory (75). This is a top down program. The second aspect is a bottom-up approach where the form of (75), or its modification, is derived from the deeper informational process physics. This is essentially the quantum gravity route. The turbulence is of course essentially a gravitational wave phenomenon and networks of 1st-order interferometers will permit spatial and time series analysis. There are a number of other gravitational anomalies which may also now be studied using such an interferometer network, and so much new physics can be expected to be uncovered.

\section{Modern Interferometers}

\subsection{Vacuum Michelson Interferometers}

Over the years vacuum-mode Michelson interferometer experiments have become increasing popular, although the motivation for such experiments appears to be increasingly unclear. The first vacuum interferometer experiment was planned by Joos [92] in 1930, but because of technical problems helium was actually used, as discussed in Sect. 10.6. The first actual vacuum experiment was by Kennedy and Thorndile [105]. The result was actually unclear but was consistent with a null effect as predicted by both the quantum-foam physics and the Einstein physics. Only Newtonian physics is disproved by such experiments. These vacuum interferometer experiments do give null results, with increasing confidence level, as for example in Refs.[105, 106, 107, 108], but they only check that the Lorentz contraction effect completely cancels the geometrical path-length effect in vacuum experiments, and this is common to both theories. So they are unable to distinguish the new physics from the Einstein physics. Nevertheless recent works $[107,108]$ continue to claim that the experiment had been motivated by the desire to look for evidence of absolute motion ${ }^{22}$, despite effects of absolute motion having been discovered as long ago as 1887. The 'null results' are always reported as proof of the Einstein formalism. Of course all the vacuum experiments can do is check the Lorentz contraction effect, and this in itself is valuable. Unfortunately the analysis of the data from such experiments is always by means of the Robertson [109] and Mansouri and Sexl formalism [110], which purports to be a generalisation of the Lorentz transformation if there is a preferred frame. However in Sect.9.2 we have already noted that absolute motion effects, that is the existence of a preferred frame, are consistent with the usual Lorentz transformation, based as it is on the restricted Einstein measurement protocol.

\footnotetext{
${ }^{22}$ The Journal Physical Review Letters has a particular penchant for publishing such works and a total aversion to works reporting experimental data giving evidence for absolute motion and its related gravitational in-flow effects.
} 
A preferred frame is revealed by gas-mode Michelson interferometer experiments, and then the refractive index of the gas plays a critical role in interpreting the data. The Robertson and Mansouri-Sexl formalism contains no contextual aspects such as a refractive index effect and is thus totally inappropriate to the analysis of so called 'preferred frame' experiments.

It is a curious feature of the history of Michelson interferometer experiments that it went unnoticed that the results fell into two distinct classes, namely vacuum and gas-mode, with recurring non-null results from gas-mode interferometers. The science historian Swenson [97] p.243 noted in 1972 that

the comparisons of expected to observed fringe shifts over the years of increasing optical sophistication still left little, though something, to be explained.

History will I think show that the physicists went to great lengths to hide that 'something', and still do so.

\subsection{Solid-State Michelson Interferometers}

The gas-mode Michelson interferometer has its sensitivity to absolute motion effects greatly reduced by the refractive index effect, namely the $k^{2}=n^{2}-1$ factor in (85), and for gases with $n$ only slightly greater than one this factor has caused much confusion over the last 115 years. So it would be expected that passing the light beams through a transparent solid with $n \approx 1.5$ rather than through a gas would greatly increase the sensitivity. Such an Michelson interferometer experiment was performed by Shamir and Fox [111] in Haifa in 1969. This interferometer used light from a He-Ne laser and used perspex rods with $L=0.26 \mathrm{~m}$. The experiment was interpreted in terms of the supposed Fresnel drag effect, which has a drag coefficient given by $b=1-1 / n^{2}$. The light passing through the solid was supposed to be 'dragged' along in the direction of motion of the solid with a velocity $\Delta \mathbf{V}=b \mathbf{v}$ additional to the usual $c / n$ speed. As well the Michelson geometrical path difference and the Lorentz contraction effects were incorporated into the analysis. The outcome was that no fringe shifts were seen on rotation of the interferometer, and Shamir and Fox concluded that this negative result "enhances the experimental basis of special relativity".

The Shamir-Fox experiment was unknown to $\mathrm{us}^{23}$ at Flinders university when in 2002 several meters of optical fibre were used in a Michelson interferometer experiment which also used a He-Ne laser light source. Again because of the $n^{2}-1$ factor, and even ignoring the Fresnel drag effect, one would have expected large fringe shifts on rotation

\footnotetext{
${ }^{23}$ This experiment was performed by Professor Warren Lawrance, an experimental physical chemist with considerable laser experience.
} 
of the interferometer, but none were observed. As well in a repeat of the experiment single-mode optical fibres were also used and again with no rotation effect seen. So this experiment is consistent with the Shamir-Fox experiment. Re-doing the analysis by including the supposed Fresnel drag effect, as Shamir and Fox did, makes no material difference to the expected outcome. In combination with the non-null results from the gas-mode interferometer experiments along with the non-interferometer experiment of DeWitte it is clear that transparent solids behave differently to a gas when undergoing absolute motion through the quantum foam. Indeed this in itself is a discovery of a new phenomenon.

The most likely explanation is that the physical Fitzgerald-Lorentz contraction effect has a anisotropic effect on the refractive index of the transparent solid, and this is such as to cause a cancellation of any differences in travel time between the two arms on rotation of the interferometer. In this sense a transparent solid medium shares this outcome with the vacuum itself.

\subsection{New Absolute Motion Detectors}

It would appear so far that either very difficult gas-mode Michelson 2nd-order interferometers, perhaps using gases with anomalously large values of $n^{24}$, or time-of-flight 1st-order experiments using atomic clocks are required to study absolute motion and related effects. Here the possibility of constructing 1st-order devices is considered. Consider first the interferometer design shown schematically in Fig.37. Fringes are produced by the interference of two beams of coherent laser light that are brought together on a screen after having passed through different media of different refractive indices, such as glass and air. If there is no Fresnel drag effect in the glass then on rotation of the device the fringes shift by an amount that is 1 st order in $v / c$

Consider a simplified analysis for which 2nd-order Fitzgerald-Lorentz contraction effects are ignored. Let the length of the glass rod have refractive index $n_{2}$ and length $L$ and the air refractive index $n_{1}$ and let the transverse distance be $d$. Then the difference in travel time for the glass and air paths is

$$
\begin{aligned}
\Delta t & =\frac{L}{\frac{c}{n_{1}}-v_{P}}-\frac{L}{\frac{c}{n_{2}}-v_{P}}+\frac{d}{\frac{c}{n_{1}}-v_{T}}+\frac{d}{\frac{c}{n_{1}}+v_{T}}, \\
& =\left(\frac{n_{1} L}{c}-\frac{n_{2} L}{c}\right)+\left(\frac{n_{2}^{2} L}{c}-\frac{n_{1}^{2} L}{c}\right) \frac{v_{P}}{c}+O\left(\frac{v_{P}^{2}}{c^{2}}\right)+O\left(\frac{v_{T}^{2}}{c^{2}}\right),
\end{aligned}
$$

where $v_{P}$ is the projection of the absolute velocity $\mathbf{v}$ onto the axis of the interferometer, and $v_{T}$ is the projection onto the transverse paths. Here the first bracket is the fixed

\footnotetext{
${ }^{24}$ The gas Perfluorobutane has $n=1.0014$ and is particularly suited to this task.
} 


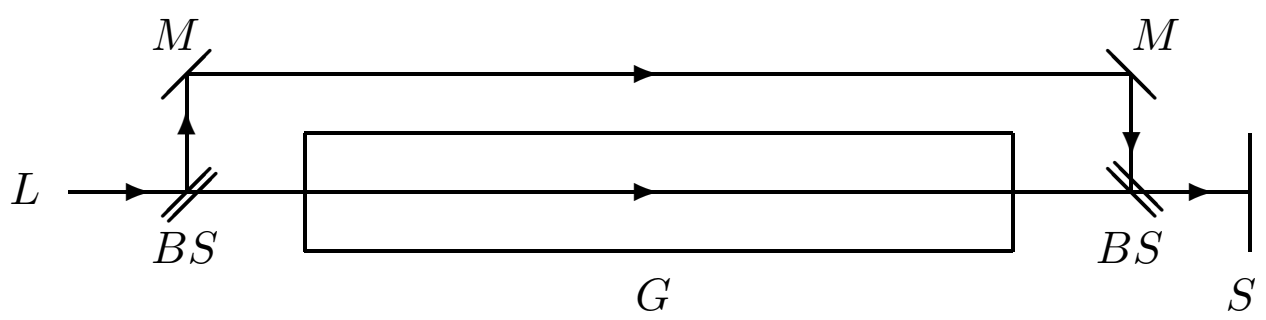

Figure 37: Schematic form of a 1st-order interferometer showing laser source (L), glass $\operatorname{rod}(\mathrm{G})$, screen $(\mathrm{S})$, mirrors $(\mathrm{M})$ and beam-splitters $(\mathrm{BS})$. The axis of the laser should ideally be orthogonal to the plane of the interferometer.

difference in travel times when $v_{P}=0$. The required effect follows from the second term, which gives the change $\delta \Delta t$ in time-difference on rotation of the device,

$$
\delta \Delta t=\left(n_{2}^{2}-n_{1}^{2}\right) t_{0} \frac{v_{P}}{c}
$$

where $t_{0}=L / c$ is the vacuum travel time for the distance $L$. Hence $\delta \Delta t$ is the change in the difference of travel times upon rotation, in which $v_{P}$ changes due to the projection effect. Hence this interferometer is a differential time-of-flight device, and essentially uses one of the beams as a timing reference beam, and so avoids the need for atomic clocks. This device can be very small. Using $n_{2}=1.5$ for the glass, $n_{1}=1$ for air, $L=0.1 \mathrm{~m}$ and a He-Ne laser, then for $v_{P}=400 \mathrm{~km} / \mathrm{s}$ we obtain $2 \delta \Delta t=0.011 \mathrm{~ns}$ corresponding to some 524 fringe shifts on a $180^{0}$ rotation, that is when $v_{P} \rightarrow-v_{P}$. This device is sufficiently sensitive that speed measurements accurate to $\pm 1 \mathrm{~km}$ should be possible. This single arm interferometer requires rotation, and this will always complicate its operation. However a static device may be constructed based on three orthogonally orientated arms. Then the fringe shifts in each arm give $\delta \Delta t_{x}, .$. and we have from (105)

$$
\mathbf{v}=\left(v_{x}, v_{y}, v_{z}\right)=\frac{c}{t_{0}\left(n_{2}^{2}-n_{1}^{2}\right)}\left(\delta \Delta t_{x}, \delta \Delta t_{y}, \delta \Delta t_{z}\right)
$$

which gives the velocity vector of absolute motion relative to the coordinate system defined by the arms. This velocity is then transformed to celestial coordinates. Such a device is ideal to study and characterise the in-flow turbulence which is an aspect of quantum gravity phenomena.

However if there is a Fresnel drag effect in transparent solids then the above analysis is changed significantly, for we then have, again ignoring 2 nd order effects,

$$
\Delta t=\frac{L}{\frac{c}{n_{1}}+b_{1} v_{P}-v_{P}}-\frac{L}{\frac{c}{n_{2}}+b_{2} v_{P}-v_{P}}+\frac{d}{\frac{c}{n_{1}}-v_{T}}+\frac{d}{\frac{c}{n_{1}}+v_{T}},
$$




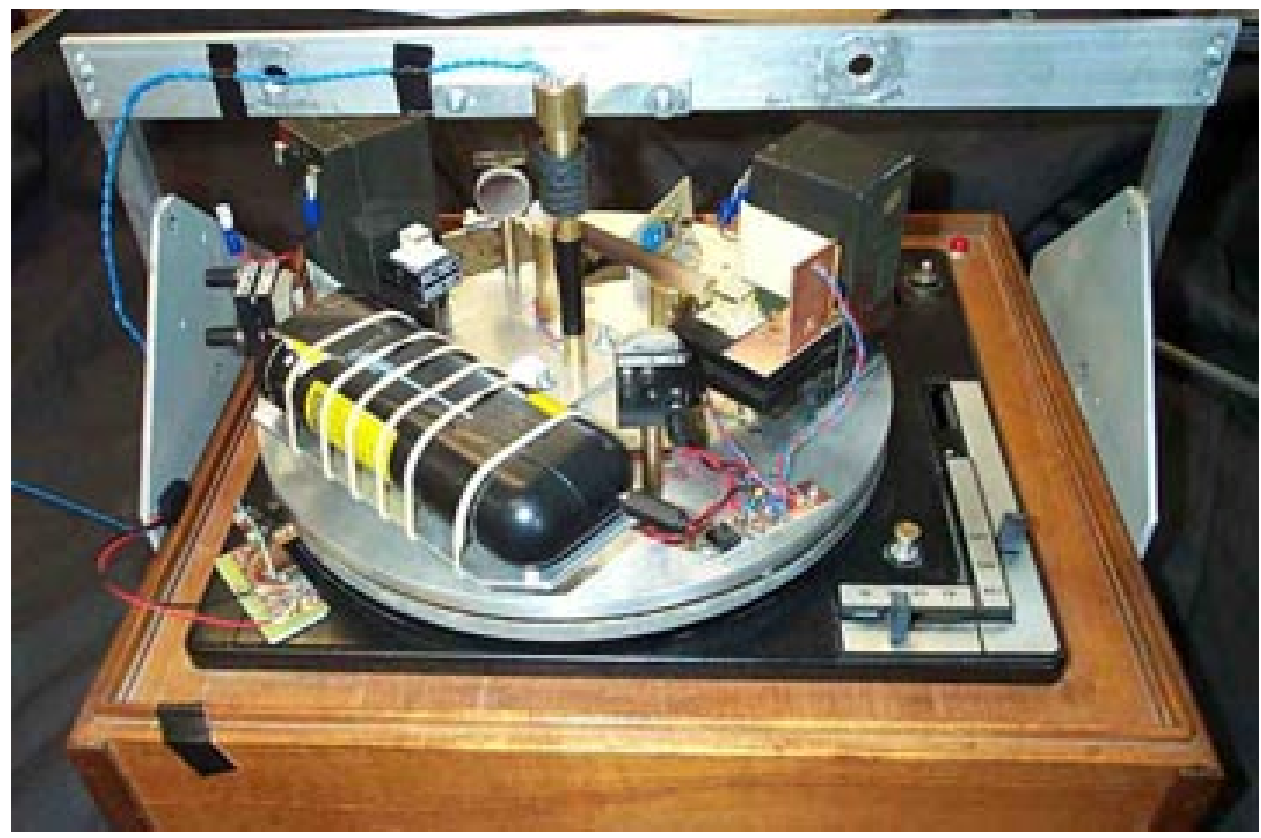

Figure 38: The Flinders experiment. The He-Ne laser is in the lower left hand corner. The glass rod is orientated from upper left to lower right. Rotations are achieved by means of the record turntable. The two rectangular black boxes are batteries.

$$
=\left(\frac{n_{1} L}{c}-\frac{n_{2} L}{c}\right)+O\left(\frac{v_{P}^{2}}{c^{2}}\right)+O\left(\frac{v_{T}^{2}}{c^{2}}\right),
$$

where $b_{i}=1-1 / n_{i}^{2}$ are the Fresnel drag coefficients, and so there is no 1st-order term in $v_{P} / c$. Then this arrangement would fail as a 1st-order detector of absolute motion. An experiment to investigate the presence or otherwise of the Fresnel drag effect in transparent solids is underway at Flinders University ${ }^{25}$, see Fig.38.

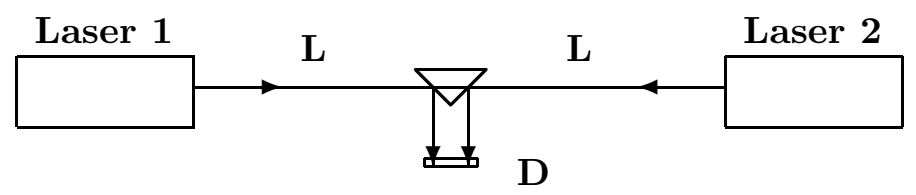

Figure 39: A 1st-order device for detecting absolute motion. Light from two identical lasers is combined and their beat frequency is detected at D.

If the Fresnel drag effect does occur in solids, and that would cause problems in understanding the DeWitte data, then a different 1st-order device is possible, as shown

\footnotetext{
${ }^{25}$ Being carried out by Warren Lawrance and Bill Drury.
} 
in Fig.39. This involves two identical antiparallel lasers. Then, as in (98), the difference in travel time to the detector is given by

$$
\Delta t_{F}=2 \frac{L}{c} \frac{v_{P}}{c}
$$

which is a 1st-order effect. The main difficulty is in the frequency stability of the two lasers. Again to avoid the need for rotations three such devices arranged orthogonally as above would be very effective.

\section{Looking Back}

\subsection{The Failure of the Einstein Postulates}

Physics is experiment based and so its theoretical proposals must be exposed to ongoing experimental challenge, although many editors of physics and other science journals don't appear to accept this notion ${ }^{26}$. The Einstein interpretation of relativistic effects was from the beginning based on a false conclusion from the now infamous Michelson-Morley experiment. That this interpretation has survived for so long and had so much influence requires careful analysis, for there have been many contributing factors apart from the politics of physics and the spurious influences of a few individuals. The psychological and sociological aspects of the creation, promotion and adoption by the physics community of the Special and General Theory of Relativity is in itself a subject that will attract great attention from the historians of science, particularly when it is realised that the history written up to now has missed the most dramatic insight of all, namely that the theory is actually falsified by experimental data, and that this evidence had been present in diversified experimental data from the very beginning. Here I will briefly lay out the amazing sequence of events that resulted in the Einstein formalism slipping through the checks of experiment, until finally it had become an unassailable edifice of theoretical physics and in popular culture a metaphor for the brilliance of the human mind. Sadly it is actually a metaphor for the exact opposite.

The search for an understanding of reality has been long and arduous. The history of this endeavour is replete with examples of the tortuous nature of this, but none more so than the confusion surrounding the explication of the nature of space and time. The Michelson-Morley experiment actually plays a key role here for it led not only to a genuine discovery, but also to much obscuration. The experiment was conceived as a technique for observing absolute motion, which is motion relative to some structural elements of

\footnotetext{
${ }^{26}$ The paper [13] reporting the discovery of the evidence for the in-flow effect in Miller's data, see Sect.11.1, and its connection to quantum gravity, was rejected by the editor of one physics journal without comment and without even allowing the paper to be peer reviewed.
} 
or within space itself. On the basis of Galilean Relativity the theory of the Michelson interferometer was layed out by Michelson, although only with the assistance of Lorentz who had to correct Michelson's analysis ${ }^{27}$. The fringe shifts seen in the experiment in 1887 were smaller than expected and so the appropriate conclusion should have been that (i) absolute motion had been detected, but (ii) that Galilean Relativity was wrong as it had predicted too large an effect. From here on and for the next 100 or so years the story becomes ever more murky.

One possible interpretation was based on the aether entrainment ideas of Stokes [112] that arose in order to explain the stellar aberration. This explanation was that the observed absolute motion of some $8 \mathrm{~km} / \mathrm{s}$, according to Galilean Relativity and for which this speed is less than the orbital speed of the earth ${ }^{28}$, was actually relative to some aetherial matter within space and which was being entrained by the motion of the earth itself through space. This explanation then had the dualism of two velocities, one of the earth's motion with respect to space and the other of the earth's motion with respect to an aether residing in space. It was this interpretation that caused Miller to do his experiment up on Mt.Wilson, the idea being that the entrainment effect would be less at higher altitudes. However the entrainment theory has never amounted to more than a distraction to the main theme and its subsequent history will not be followed here $^{29}$.

On the incorrect basis that there had been no fringe shifts Fitzgerald and Lorentz independently offered the explanation that the arm parallel to the direction of absolute motion had been shortened as a physical effect of the motion, and that the amount of contraction was such as to exactly cancel the geometrical effect that Michelson and Lorentz had shown was expected on the basis of Galilean Relativity. So we have here another error, as the actual fringe shifts were not null, only small. Nevertheless this contraction effect turned out to be fundamentally correct, although Einstein was to put a different and now incorrect interpretation upon it. At the same time Larmor suggested that a time dilation effect would occur for actual physical clocks. In 1887 Voigt [113] had already proposed what is now known as the Lorentz transformation ${ }^{30}$. As argued herein

\footnotetext{
${ }^{27}$ Michelson had neglected that in Galilean Relativity the propagation times in the transverse arm, as well as the longitudinal arm, is affected by absolute motion.

${ }^{28}$ As well the magnitude of the fringe shifts at 12 noon and $6 \mathrm{pm}$ had the wrong dependence on solar time in order to be explained as being caused by the orbital motion of the earth about the sun, see Fig.15. This meant of course that the fringe shifts were caused by an absolute motion in a direction different to the orbital velocity direction.

${ }^{29}$ In searching for evidence to support this entrainment explanation James DeMeo travelled to Cleveland in 2002 in an attempt to locate the Miller interferometer data, and succeeded!

${ }^{30}$ Later Lorentz suggested that the 'Lorentz Transformation' be called the 'Transformation of Relativity'.
} 
this is now understood to be a mapping between measurements made by observers using rods and clocks for which the corrections for the length contraction and time dilation effects have not been made. In essence the Lorentz transformation deals with 'raw' observational data. Of course these two effects are real and as such represent a significant discovery about the dynamics of space itself, although following Einstein this was not the way they were finally seen. Of course it would be bizarre if, for example, a physicist did not correct for temperature effects on a measuring rod in measuring lengths, but this is exactly what has happened to length and time measurements within the Einstein formalism: the physical effects are asserted to be in principle not correctable.

As a consequence of these developments there emerged the Lorentzian interpretation of these relativistic effects. Lorentzian Relativity (LR) asserts that motion through space causes physical effects upon rods and clocks, and that measurements with these by observers in different states of absolute motion could be related by means of a linear mapping - the Lorentz transformation. What was to cause much confusion later was that the Lorentz transformation made no mention of the absolute velocity of the two observers, it only contains reference to their relative velocity, and so the effects of the absolute motion had become hidden as far as this mapping is concerned. However it remains valid that one of the possible observers is actually at rest with respect to the actual physical space, and that the Lorentz transformation is not the full story.

It is at this stage that Albert Einstein and his wife Mileva Maric-Einstein ${ }^{31}$ enter the story, and their individual roles in that story have never been resolved. Essentially their contribution was to formalise some of what had been discovered by the earlier investigators $^{32}$. Rather than acknowledging and building upon that work the relativistic effects were to be explained by the postulates (1) and (2) of (58). This is the Einsteinian Relativity (ER) formalism. In this the history of the subject until then was completely turned around and from the postulates the Lorentz transformation was derived. This had a disastrous effect on the future of physics as from then on physicists, with sloppy thinking, were led to believe that since the Lorentz transformation had been derived from the postulate that denied the existence of absolute motion, then absolute motion must necessarily be incompatible with relativistic effects. It must be the case that we have one or the other. Both of these postulates were already in conflict with the actual data of the Michelson-Morley experiment. Although the Einsteins were aware of this experiment ${ }^{33}$ it is not clear whether they were aware that fringe shifts of the correct form had in fact been observed.

The major and fundamental difference between LR and ER is that in LR absolute

\footnotetext{
${ }^{31}$ Mileva studied physics and mathematics at the ETH in Switzerland.

${ }^{32}$ For a revealing analysis of the role of the Einsteins in the development of relativity see [114].

${ }^{33}$ As revealed in the letters A. Einstein later wrote to Shankland.
} 
motion is meaningful and measurable, while in ER it is not meaningful and hence not measurable. The ER formalism is based on axiomatising the feature of the Lorentz transformation that the absolute velocities of each observer do not play a role. Of course it is simple to experimentally distinguish between LR and ER as they have opposite predictions regarding the detection of absolute motion. Unfortunately for the history of physics the Michelson interferometer is not very sensitive to the effects of absolute motion as discussed elsewhere in this work. Indeed the Michelson interferometer is unsuited to this task. Its major use is actually in checking the accuracy of the FitzgeraldLorentz contraction effect, and to do this it must operate in vacuum mode. To detect absolute motion it must operate in gas mode. Because this was not understood right through the twentieth century it was assumed that as better and better vacuum Michelson interferometers were developed and that no evidence of absolute motion was forthcoming then the ER formalism must be the correct one. In fact there had been gas-mode interferometer experiments such as Miller's that had most clearly detected the effects of absolute motion, but they were rejected for totally spurious arguments.

One consequence of the Einstein postulates and the ER formalism was the introduction and acceptance of the spacetime construct, and the assertion that only this had physical or ontological significance and that the older separate concepts of time as process and space as an entity of some sort were abandoned. In the spacetime construct the notion of change is removed. All that remained was a bizarre geometrical modelling of time. The spacetime construct is an artifact of the Lorentz transformation and as such lacks the dynamics that the effects of absolute motion revealed. Spacetime is itself a totally static geometrical structure upon which the whole edifice of twentieth century physics was built. It certainly contains some truth as the Lorentz transformation upon which it is founded does encode the consequences of actual physical effects, and so the validity of much of twentieth century theoretical physics is limited to that which is compatible with these effects but which explicitly denies the underlying dynamics.

The ongoing confusion in physics has led to the belief that the small effects of absolute motion seen in interferometer experiments are incompatible with relativistic effects and so the effects must be caused by spurious experimental imperfections and hence the ER formalism is confirmed. Indeed most so-called experimental confirmations of the ER formalism are really nothing more than demonstrations of relativistic effects, and as such are equally compatible with LR.

However worse was to come. Eventually through the work of Hilbert and Einstein a mathematical generalisation of the ER formalism to include gravitational effects was produced. In this gravity is understood to be a curvature of the previously flat spacetime construct. This generalisation was designed to reduce to the flat spacetime construct in the absence of matter, while in the case of low velocities it was meant to reduce to New- 
tonian gravity, at least as far as its predictions went, although it clearly had a different ontology. There are now various experimental and observational demonstrations of the supposed efficacy of this generalised spacetime formalism, but as noted previously none of these actually support the formalism. In the only cases where the formalism has been checked the metric of the curved spacetime turns out to be flat and identically equivalent to the in-flow formulation of Newtonian gravity. So to this extent the generalisation is actually untested. The so called demonstrations amount to nothing more than relativistic effects, using (37), where the absolute velocity of the object's motion relative to absolute space is manifest, and which may possess an inhomogeneous flow as a manifestation of gravity.

\subsection{The Shankland Paper: 1955}

As previously discussed the Miller experiment has now been strongly confirmed by other experiments, including two which were not even interferometer experiments. All agree on the direction and speed of absolute motion that Miller was the first to discover. As well his data now provides experimental evidence for an explanation of gravity different from those of both Newton and Einstein. So the Miller experiment was one of the most significant physics experiments done in the twentieth century. We know that Einstein was very concerned about the Miller experiments for as he noted in a letter to Robert Millikan in June 1921,

I believe that I have really found the relationship between gravitation and electricity, assuming that the Miller experiments are based on a fundamental error. Otherwise, the whole relativity theory collapses like a house of cards. (in Clark[99] 1971, p.328)

So why were Miller's experiments rejected? Part of the answer is that by the time Miller began reporting his results the Einstein interpretation of relativistic effects had become the prevailing paradigm, as well as the belief that the Michelson-Morley experiment had indeed shown a 'null effect'. As well no one had noticed that the small effect actually present in these gas-mode interferometer experiments was related to the type of gas present, and that furthermore if the gas was removed only then did the small effect truly go away. That was all bad enough showing as it did a disregard for the scientific method. Miller's data was clearly revealing the failure of the Einstein formalism, as Einstein was himself very much aware. Suggestions that the effects were temperature effects had of course led to intensive investigation by Miller over many years, and even Einstein had been pressing this explanation. In responding to Einstein Miller wrote that

The trouble with Professor Einstein is that he knows nothing about my results...He ought to give me credit for knowing that temperature differences 
would affect the results. He wrote to me in November suggesting this. I am not so simple as to make no allowance for temperature.

(Cleveland Plain Dealer, January 27, 1926)

However worse was to come for Shankland, who had assisted Miller in the analysis of his data, and after prior consultations with Einstein, produced a critique [90] of the Miller results in which Shankland claimed, 14 years after Miller's death, that the Miller fringe shifts were due to statistical fluctuations in the readings of the fringe positions, and other systematic effects were ascribed to local temperature conditions. Shankland wrote [90] that

Miller's extensive Mt.Wilson data contained no effect of the kind predicted by the aether theory and, within the limitations imposed by the local disturbances, are entirely consistent with a null result at all epochs during a year.

Miller however had already noted that at no time did he ever observe any periodic effects related to local civil time as would be the case if the sun's heating effect were present. On the contrary Miller's results show very clearly that the features in the data correlate with sidereal time.

An extensive review of the Shankland paper has recently been written by DeMeo [98] where various deficiencies of that paper are noted. The claim by Shankland that the periodic effects were temperature effects caused by the heating of the sun had been thoroughly investigated by Miller, and are known to be invalid. Shankland also dismissed Miller's results on the grounds that there were significant fluctuations present in the data. Of course Shankland was assuming, without investigation, that these fluctuations were not evidence of a real physical effect. We now believe that these fluctuations are in fact evidence of turbulence in the gravitational in-flow. So Shankland's dismissal of the Miller experiment was an enormous blow to the progress of physics in the twentieth century.

Einstein was deeply appreciative of Shankland's paper and its rebuttal of the effects that Miller had reported. In 1954 Einstein wrote to Shankland:

I thank you very much for sending me your careful study about the Miller experiments. Those experiments, conducted with so much care, merit, of course, a very careful statistical investigation. This is more so as the existence of a not trivial positive effect would affect very deeply the fundament of theoretical physics as it is presently accepted. You have shown convincingly that the observed effect is outside the range of accidental deviations and must, therefore, have a systematic cause having nothing to do with 'ether wind', but with differences of temperature of the air traversed by the two light bun- 
dles which produce the bands of interference.

(Shankland [115], p.2283)

\section{Conclusions}

Process Physics has been shown to be a major development in the modelling of reality. After some 400 years, during which physics was founded on a geometrical model of time, we now have a new non-geometrical process modelling of time that matches all the aspects of the phenomena of time. This model of time is part of an information-theoretic approach to comprehending reality. It was inspired by the logic of the limitations of the logic of formal syntactical information systems discovered by Gödel. The structural randomness, discovered by Chaitin, beyond the Gödel boundary in such systems was generalised to randomness in a time-like processing system by introducing the concept of self-referential noise. In this way we model the idea that there are limits to the information content within the processing information-theoretic system and in reality itself. Most significant is that this system entails the idea of semantic information, which is information that is generated and recognised within the system itself. This is in sharp contrast to the syntactical information-theoretic approach that has been the core concept in the traditional modelling of reality from its beginnings, though rarely acknowledged as such. As well evidence of a processing information-theoretic system was exposed as being at the base of the quantum field theory modelling of quantum matter, by means of the stochastic 'quantisation' formalism.

Having set up a processing information-theoretic system in which all information is internally generated and recognised we have explored in considerable detail the nature of the emergent information and shown that, to the extent currently available, that the system generates phenomena that exhibit a remarkable likeness to those aspects of reality that physicists have so carefully revealed over past centuries. These include:

1. The emergence of elements of geometry by way of the gebits.

2. The self-linking of these gebits to form a fractal processing system displaying quantum-foam behaviour that entails both space and quantum matter embedded in that space. So we have a quantum behaviour emergent within a system in which no quantum behaviour had been present. Thus we have a derivation of the logical necessity of quantum behaviour from the processing information-theoretic system. The key concept of self-referential noise is central to this emergence.

3. The quantum description of space displays ongoing non-local collapse of its quantum structure so that a classical 3D-space is effectively present at a higher level. 
This space is growing in size over time and this matches the observed behaviour of our universe.

4. A formalism to encode the emergent behaviour of the quantum 'matter' within the system was developed. Here 'matter' corresponds to topologically stabilised defects within the spatial network, and it was argued that these topological defects display flavour and hidden colour degrees of freedom. The spin $1 / 2$ formalism is also emergent.

5. The quantum 'matter', like space itself, is fundamentally non-local, and the collapse mechanism that is known to happen within quantum system upon measurements is now explained, and the randomness of that collapse is seen to be yet another manifestation of the self-referential noise. This ongoing and internal collapse process is responsible for the emergence of classical behaviour, which explains why at a macroscopic level our reality is discrete and hard, and very different from the underlying information-theoretic substratum.

6. The unification of space and quantum in a process system led to an explanation of inertia and gravity, with the later displaying the equivalence principle. It was argued that matter effectively acts as a sink for the information patterns in the quantum foam that forms the spatial degrees of freedom. Then gravity manifests as a net in-flow of that spatial information into matter.

7. The motion of matter through this spatial system is expected to display the relativistic effects of length contractions and time dilations. As well because space is fundamentally different from time absolute motion is a key prediction of process physics. It was shown that the spacetime construct is not an aspect of reality, but at best only a useful mathematical tool. Fundamentally the Lorentzian interpretation of relativistic effects is seen to arise within process physics.

8. The new theory of space and gravity was investigated in considerable detail. It was shown that Newtonian gravity could be written in the mathematical language of a fluid flow system. The Hilbert-Einstein General Relativity spacetime formalism was shown to be completely equivalent to this Newtonian flow mathematics in all cases where the spacetime formalism had been checked, namely the Schwarzschild metric for spherically symmetric systems. So the famous tests of General Relativity had in fact not tested the GR formalism, but merely the Newtonian gravitational formalism.

9. A generalisation of the Newtonian gravitational flow formalism was proposed which will give different gravitational effects when the matter system is not spherically 
symmetric. This system or some variation of it appears to offer an explanation for the effects seen in spiral galaxies which have been misinterpreted as being caused by dark matter. Rather these effects are seen as indicators of the failure of Newtonian gravity, a formalism that had been inspired by the overly special nature of the solar system, namely its strong spherical symmetry.

10. The failure of Newtonian gravity is expected to cause difficulties in measuring $G$, as has been the case.

11. Process Physics predicts that absolute motion is meaningful and measurable, in sharp contrast to the Einstein assumptions. A detailed investigation of absolute motion experiments revealed that for over 100 years many of these experiments had indeed detected absolute motion. These include the original experiment by Michelson and Morley. The analysis of the data from this influential experiment was totally bungled.

12. By the time Miller, some 40 years later, had figured out how to avoid a repetition of the Michelson-Moley errors and had convincingly established the existence of absolute motion effects and had determined a speed and direction of motion of the solar system, the physics community was firmly in the erroneous mindset that Einstein had promulgated. One consequential devastating misunderstanding, which is still prevalent, is that absolute motion is totally incompatible with relativistic effects. This arose from Einstein's derivation of these relativistic effects by denying absolute motion. So most physicists are of the belief that the many examples of relativistic effects imply that absolute motion is impossible. Conseqentially any experimental evidence to the contrary has been suppressed over the last 100 years. This has had a devastating effect on physics.

13. It was shown that the Michelson interferometer can detect absolute motion when operated in gas mode, and many examples of such experiments were given. All these experiments are in agreement.

14. Several non-interferometer experiments were also analysed and the remarkable coaxial cable experiment by DeWitte in 1991 was shown to be in agreement with the interferometer experiments. These comparisons show that the FitzgeraldLorentz contraction is a real physical process.

15. A feature of the Miller interferometer data was that with the new gas-mode effects now understood, a re-analysis of the Miller data revealed the in-flow of space past the earth towards the sun. So this old and ridiculed experiment was seen to provide evidence in support of the new theory of gravity. These data as well as those of the 
DeWitte experiment suggested that the in-flow displayed turbulence, as expected of the new theory.

16. It was shown that the new theory of gravity is consistent with the operation of the Global Positioning System (GPS), but that the system could be used to study the new gravitational wave phenomena.

17. The direction of motion of the solar system through space is distinctly different from the direction of motion of the solar system with respect to the Cosmic Microwave Background. This is interpreted as evidence for the in-flow of space into the Milky Way, and is further evidence in support of the new theory of gravity.

18. Various bench-top devices for detecting absolute motion and the new gravitational wave effect were discussed.

19. The various observations of absolute motion over the last 115 years prove conclusively that the Einstein formalism is incorrect. The belief that experiments had confirmed that formalism are based on ongoing misunderstandings. In all cases the experiments were confirming relativistic effects that have nothing to do with the Einstein formalism, and which predated that formalism. It is the original Lorentz Relativity that experiments have been confirming.

20. The emergent unification within process physics of quantum behaviour for both 'matter' and space, and displaying gravitational phenomena, means that we have a quantum theory of gravity.

21. The velocity field turbulence present in the Miller and DeWitte data clearly amount to the observation of gravitational waves. But this observed phenomenon is very different from those gravitational waves predicted by the Hilbert-Einstein GR formalism. As that formalism is now demonstrably falsified by experiment there is no reason to believe that these GR gravitational waves even exist.

Finally the successes of process physics imply that we should give consideration to its foundational assumptions, and one is that reality is a non-local experiential information system. One way to interpret this is that reality has a primitive form of self-awareness. In increasingly more complex systems such as biological systems this self-awareness may manifest as consciousness. This is a conjecture for further careful consideration, see $[128,129]$.

Process Physics is clearly a model of reality that implements many of the ideas that have been considered within Process Philosophy, particularly the work of Whitehead. The convergence of this physics and this philosophy represents an exciting development 
and a unification of not only various areas of physics but also of many other areas of human intellectual endeavours. This all points to an exciting future.

\section{Acknowledgments}

This paper is dedicated to the memory of Dayton C. Miller of the Case School of Applied Science, Cleveland, Ohio. A special acknowledgement to James DeMeo who travelled to the Physics Department of the Case Western Reserve University in 2002 resulting in the discovery that the extensive and valuable Miller data still existed. Thanks to Christopher Klinger, Kirsty Kitto, Susan Gunner, John Wheldrake, Hank Keeton, Tim Eastwood, Horacio Velasco, Paul Dryga, Jon Opie, Ron Burman, Igor Bray, Glen Deen, and Lance McCarthy. Thanks to Katie Pilypas for running the codes to fit the Miller data. Particular thanks to Professor Warren Lawrance for the ongoing exchanges of ideas as we explored new ways to experimentally test the new physics, and to Bill Drury who enthusiastically constructed the apparatus. Much inspiration has come from the regular meetings of Pamela Lyon's Intersections Group with members Pamela, Theresa Hickey, David Scrimgeour and myself. Over many meetings we have explored the Buddhist and Western Process Philosophy insights into our comprehension of reality and how those insights are manifested not only in the new Process Physics but also in various aspects of biological systems. Thanks to Hank Keeton and Dan Dombrowski for proof reading this document. Finally a special thanks to my wife Krystyna.

\section{Appendices}

\subsection{Gebit Connectivity}

The probability that a connected random graph with $N_{g}$ vertices has a depth structure $D_{0}, D_{1}, \ldots, D_{L}$ was given in (13) and there it was shown that it leads to the concept of emergent geometry via the gebit concept. Eqn.(13) was first derived by Nagels [35]. The notes here are adapted from those by Christopher Klinger.

Consider a set of $\mathrm{M}$ nodes with pairwise links arising with probability $p \ll 1$. The probability of nonlinking is then $q=1-p$. We shall term linked nodes as being 'adjacent', though the use of this geometric language is to be justified and its limitations determined. The set $M$ will be partitioned into finite subsets of mutually disconnected components, each having $N_{i}$ nodes which are at least simply connected - that is, each $N_{i}$ may be described by a non-directed graph.

Consider one of these components, with $N=N_{i} \gg 1$, and choose one vertex to be the 'origin'. We will determine the probable distribution of vertices in this component as 
measured by the depth structure of a minimal spanning tree. See Fig.5c for the definition of depth structure. Let $D_{k}$ be the number of vertices at a distance $k$ from the origin, then $D_{0}=1$ is the origin, $D_{1}$ is the number of adjacent vertices or nearest neighbours to the origin, and $D_{2}$ is the number of next nearest neighbours and so on. Then, since $N$ is finite, there is a maximum distance $L$ on the graph and $D_{L}$ is the number of vertices at this maximum distance from the origin. There is then the constraint

$$
\sum_{k=0}^{L} D_{k}=N
$$

and also

$$
\left\{\begin{array}{l}
D_{0}=1, \\
D_{k}>0, \quad 0 \leq k \leq L \\
D_{k}=0, \quad k>L
\end{array}\right.
$$

To calculate the probability for the distribution $\left\{D_{k}: 0 \leq k \leq N, \sum_{k=0}^{N-1} D_{k}=N\right\}$ we require:

1. the probability for the number $D_{1}$ of nearest neighbours (i.e. those vertices at unit distance from the origin) is $p^{D_{1}}$, which may be written as $(1-q)^{D_{1}}=\left(1-q_{0}^{D_{0}}\right)^{D_{1}}$, since $D_{0}=1$;

2. the probability for the next nearest neighbours, $D_{2}$, is obtained by considering that any vertex at this level is

(a) adjacent to at least one point at unit distance from the origin;

(b) not adjacent to the origin itself.

Condition (b) is easily obtained since it occurs with probability $q=1-p$ so there is a factor of $q^{D_{2}}$ for this.

Condition (a) may be obtained by first considering the counter argument, i.e. that the vertex is not adjacent to any of the $D_{1}$. This has probability $q^{D_{1}}$. Thus the probability that it is adjacent to at least one of the $D_{1}$ is just $1-q^{D_{1}}$. So there is an overall factor of $\left(1-q^{D_{1}}\right)^{D_{2}}$ for this condition.

Hence, the probability of obtaining $D_{2}$ is the product of these two factors, i.e.

$$
\operatorname{prob}\left(D_{2}\right)=\left(1-q^{D_{1}}\right)^{D_{2}} q^{D_{2}}
$$

3. the probability for $D_{3}$, those vertices at distance $k=3$ from the origin, is similarly defined by the requirements that a vertex in $D_{3}$ is 
(a) adjacent to least one vertex in $D_{2}$;

(b) not adjacent to any vertex in $D_{1}$;

(c) not adjacent to the origin.

Condition (a) is argued precisely as the corresponding condition in item 2 above, i.e. it provides a factor $\left(1-q^{D_{2}}\right)^{D_{3}}$.

Condition (b) is expressed as $q^{D_{1}}$, thus providing the factor $\left(q^{D_{1}}\right)^{D_{3}}$.

Conditioned (c) is satisfied simply by the factor $q^{D_{3}}$, which may be written as $\left(q^{D_{0}}\right)^{D_{3}}$ since $D_{0} \equiv 1$. Hence the probability of obtaining $D_{3}$ is

$$
\left(1-q^{D_{2}}\right)^{D_{3}}\left(q^{D_{1}}\right)^{D_{3}}\left(q^{D_{0}}\right)^{D_{3}}=\left(q^{D_{0}+D_{1}}\right)^{D_{3}}\left(1-q^{D_{2}}\right)^{D_{3}}
$$

4. for vertices at a distance $i+1$ from the origin, induction on the previous results gives

$$
\operatorname{prob}\left(D_{i+1}\right)=\left(q^{\sum_{j=0}^{i-1} D_{j}}\right)^{D_{i+1}}\left(1-q^{D_{i}}\right)^{D_{i+1}}
$$

So the probability $P$ for the depth distribution is the probability of obtaining a particular set $\left(D_{1}, D_{2}, \cdots, D_{L}\right)$ which is

$$
P=p^{D_{1}} \prod_{i=1}^{L-1}\left(q^{\sum_{j=0}^{i-1} D_{j}}\right)^{D_{i+1}}\left(1-q^{D_{i}}\right)^{D_{i+1}}
$$

Note that vertices may be permuted between the sets of vertices at different distances. That is, the same magnitudes for each $D_{k}$ could be obtained by many other possible configurations which result from a relabelling of the graph. First, there are $(N-1)$ ! ways of relabelling the graph once the choice of origin has been fixed so there are $(N-1)$ ! ways of obtaining the same $P$, where the depth structure given by $\left(D_{1}, D_{2}, \cdots, D_{L}\right)$ is identical. Second, the number of instances of a particular shape irrespective of labelling (beyond the choice of origin) is given by the product $D_{1} ! D_{2} ! \cdots D_{L} !$.

Hence there are $\frac{(N-1) !}{D_{1} ! D_{2} ! \cdots D_{L} !}$ ways of obtaining a graph (from a fixed origin) with a particular depth structure and therefore, the probability for a specified shape with $\mathrm{N}$ given and the origin arbitrarily chosen, that is, the probability distribution, is

$$
\mathcal{P}=\frac{(N-1) !}{D_{1} ! D_{2} ! \cdots D_{L} !} p^{D_{1}} \prod_{i=1}^{L-1}\left(q^{\sum_{j=0}^{i-1} D_{j}}\right)^{D_{i+1}}\left(1-q^{D_{i}}\right)^{D_{i+1}}
$$

which is (13). 


\subsection{Fresnel Drag}

Fresnel drag is the observed effect in which motion of a transparent medium partially drags the light, so that instead of the speed being $V=c / n$ it is increased to the extent given in (117). The explanation for this effect is yet to be determined, although over the years a variety of explanations have been produced. Fresnel in considering the possible nature of the aether ${ }^{34}$ assumed that the density of the aether in a transparent material is proportional to the square of the refractive index $n: \rho_{m} / \rho_{v}=n^{2}$. When the matter moves through the aether that part of the aether in excess of the vacuum value is carried along: $\rho_{m}-\rho_{v}=\left(n^{2}-1\right) \rho_{v}$. Then the centre of mass of the aether moves with speed

$$
v_{c m}=\frac{\left(n^{2}-1\right) v+1.0}{\left(n^{2}-1\right)+1}=\left(1-\frac{1}{n^{2}}\right) v
$$

where $v$ is the velocity of the matter. This velocity is added to the velocity $c / n$ of light in the body, so that

$$
V=\frac{c}{n}+\left(1-\frac{1}{n^{2}}\right) v
$$

and $f=\left(1-1 / n^{2}\right)$ is known as the Fresnel drag coefficient. Fizeau confirmed this result using moving water in 1851. Michelson and Morley in 1886 confirmed the result using transparent liquids such as water, carbon disulfide and others with a high $n$.

Stokes in 1842 gave another derivation assuming that the aether was a compressible but conserved fluid. If the aether has an apparent speed $v$ with respect to the transparent matter then let $v^{\prime}=(1-\kappa) v$ be the drag speed. If the aether density is $\rho=\rho_{0}$ in vacuum, then the density is $\rho^{\prime}=n^{2} \rho_{0}$ in the matter. If the aether is conserved then $\rho_{0} v=\rho^{\prime} v^{\prime}=n^{2}(1-\kappa) \rho_{0} v$, and so $\kappa=1-1 / n^{2}$. Lorentz in 1892 gave another derivation based on the microstructure of the moving transparent medium.

The Fresnel drag may also be derived as a consequence of the relativistic velocity transformation, which follows from the Lorentz transformation. The mathematics of this is straightforward. The Lorentz transformation gives the velocity addition rule:

$$
V=\frac{v+v^{\prime}}{1+\frac{v v^{\prime}}{c^{2}}} .
$$

The meaning of this is that if an observer $O^{\prime}$ measures a light wave moving at speed $v^{\prime}$, and $O^{\prime}$ is moving with speed $v$ relative to observer $O$, then (118) gives the speed $V$ of that light wave according to $O$. Then for $v^{\prime}=c / n$ we obtain (117). However the meaning of this mathematical derivation is not so clear as the argument does not coincide with the actual physical situation. The Fresnel drag effect is that if an observer measures

\footnotetext{
${ }^{34}$ Adapted from notes by G.F. Smoot
} 
the speed of the light wave to be $c / n$ when the matter is at rest with respect to that observer, then the speed measured by the same observer when the matter is moving at speed $v$ is given by (117).

\subsection{Michelson Interferometer Analysis - no Fresnel Drag}

Here is a simplified analysis of the Michelson interferometer when the Fresnel drag is neglected and when for simplicity the arms are parallel/orthogonal to the direction of motion, as shown in Fig.11. Let the arms have lengths $L_{1}$ and $L_{2}$ when at rest. The Fitzgerald-Lorentz effect is that the arm $A B$ parallel to the direction of motion is shortened to

$$
L_{\|}=L_{1} \sqrt{1-\frac{v^{2}}{c^{2}}}
$$

by absolute motion. We consider the case when the apparatus is moving at speed $v$ through space, and that the photon states travel at speed $V=c / n$ relative to the quantum-foam which is space, where $n$ is the refractive index of the gas and $c$ is the speed of light, in vacuum, relative to the space. Let the time taken for $\psi_{1}$ to travel from $A \rightarrow B$ be $t_{A B}$ and that from $B \rightarrow A$ be $t_{B A}$. In moving from the beamsplitter at $A$ to $B$, the photon state $\psi_{1}$ must travel an extra distance because the mirror $B$ travels a distance $v t_{A B}$ in this time, thus the total distance that must be traversed is

$$
V t_{A B}=L_{\|}+v t_{A B}
$$

Similarly on returning from $B$ to $A$ the photon state $\psi_{1}$ must travel the distance

$$
V t_{B A}=L_{\|}-v t_{B A}
$$

Hence the total time $t_{A B A}$ taken for $\psi_{1}$ to travel from $A \rightarrow B \rightarrow A$ is given by

$$
\begin{aligned}
t_{A B A}=t_{A B}+t_{B A} & =\frac{L_{\|}}{V-v}+\frac{L_{\|}}{V+v} \\
& =\frac{L_{\|}(V+v)+L_{\|}(V-v)}{V^{2}-v^{2}} \\
& =\frac{2 L_{1} V \sqrt{1-\frac{v^{2}}{c^{2}}}}{V^{2}-v^{2}} .
\end{aligned}
$$

Now let the time taken for the photon state $\psi_{2}$ to travel from $A \rightarrow C$ be $t_{A C}$, but in that time the apparatus travels a distance $v t_{A C}$. Pythagoras' theorem then gives

$$
\left(V t_{A C}\right)^{2}=L_{2}^{2}+\left(v t_{A C}\right)^{2}
$$


which gives

$$
t_{A C}=\frac{L_{2}}{\sqrt{V^{2}-v^{2}}}
$$

and including the return trip $C \rightarrow A, t_{C A}=t_{A C}, t_{A C A}=t_{A C}+t_{C A}$ results in

$$
t_{A C A}=\frac{2 L_{2}}{\sqrt{V^{2}-v^{2}}},
$$

giving finally for the time difference for the two arms

$$
\Delta t_{0}=\frac{2 L_{1} V \sqrt{1-\frac{v^{2}}{c^{2}}}}{V^{2}-v^{2}}-\frac{2 L_{2}}{\sqrt{V^{2}-v^{2}}} .
$$

Now let the two arms be rotated through $90^{\circ}$, so that the roles of $L_{1}$ and $L_{2}$ become interchanged. Then the difference in travel time becomes

$$
\Delta t_{90}=\frac{2 L_{1}}{\sqrt{V^{2}-v^{2}}}-\frac{2 L_{2} V \sqrt{1-\frac{v^{2}}{c^{2}}}}{V^{2}-v^{2}} .
$$

Then on rotation the change in difference of the travel times, which gives the observed fringe shift, is given by

$$
\Delta t=\Delta t_{0}-\Delta t_{90}=\frac{2\left(L_{1}+L_{2}\right) V \sqrt{1-\frac{v^{2}}{c^{2}}}}{V^{2}-v^{2}}-\frac{2\left(L_{1}+L_{2}\right)}{\sqrt{V^{2}-v^{2}}},
$$

so that the effective arm length is $L=\left(L_{1}+L_{2}\right) / 2$, which is the average. For $V=c / n$ appropriate for no Fresnel drag, and for $v \ll c$ we obtain for $n \approx 1^{+}$

$$
\Delta t=2 \frac{\left(n^{2}-1\right) L}{c} \frac{v^{2}}{c^{2}}+O\left(v^{4}\right) .
$$

\subsection{Michelson Interferometer Analysis - with Fresnel Drag}

Now consider the derivation in Sect.16.3 when the Fresnel drag is included. Consider the Michelson interferometer operating in a gas which is moving with the interferometer at speed $v$. The motion of the gas relative to space results in a Fresnel drag effect. For simplicity consider only the cases when the arms are parallel/orthogonal to the direction of motion, as shown in Fig.11. Let the arms have equal lengths $L$ when at rest. The Fitzgerald-Lorentz relativistic effect is that the arm $A B$ parallel to the direction of motion is shortened to

$$
L_{\|}=L \sqrt{1-\frac{v^{2}}{c^{2}}}
$$


by absolute motion, while the length $L$ of the transverse arm is unaffected. We work in the absolute rest frame. Consider the photon states in the $A B$ arm. They travel at speed $V=c / n \pm b v$ relative to the quantum-foam which is space, where $n$ is the refractive index of the gas and $c$ is the speed of light in vacuum and relative to the space. Here $b=1-1 / n^{2}$ is the Fresnel drag coefficient which is well established experimentally. The motion of the gas through the quantum foam slightly 'drags' the light. The effect on the speed is $\pm b v$ depending on the direction of the light relative to the direction of absolute motion. Then the total travel time $t_{A B A}$ is

$$
\begin{aligned}
t_{A B A}=t_{A B}+t_{B A} & =\frac{L_{\|}}{\frac{c}{n}+b v-v}+\frac{L_{\|}}{\frac{c}{n}-b v+v} \\
& =\frac{2 L n}{c} \sqrt{1-\frac{v^{2}}{c^{2}}} \frac{1}{1-\frac{v^{2}}{n^{2} c^{2}}} .
\end{aligned}
$$

For the orthogonal arm we have by Pythagoras' theorem

$$
\left(V t_{A C}\right)^{2}=L^{2}+\left(v t_{A C}\right)^{2}
$$

The speed $V$ of light travelling from $A$ to $C$ (and also from $C$ to $A$ ) is

$$
V=\frac{c}{n}+b v \cos (\alpha)
$$

where $\alpha$ is the angle of the transverse light path to the direction of motion of the interferometer, as shown in Fig.11, and is given by

$$
\cos (\alpha)=\sqrt{1-\frac{L^{2}}{(V t)^{2}}} .
$$

Solving (135), (136) and (137) for $V$ we obtain

$$
V=\frac{1}{2}\left(\frac{c^{2}}{n^{2}}+\sqrt{\frac{c^{2}}{n^{2}}+4 b v^{2}}\right) .
$$

Then (135) gives $t_{A C}$, and we obtain, with $t_{A C A}=t_{A C}+t_{C A}=2 t_{A C}$, and for $v \ll c$

$$
\Delta t=2\left(t_{A B A}-t_{A C A}\right)=-2 \frac{\left(n^{2}-1\right)\left(2-n^{2}\right) L}{n c} \frac{v^{2}}{c^{2}}+O\left(v^{4}\right),
$$

for the change in relative travel times when the apparatus is rotated through $90^{\circ}$. The factor of 2 arises because then the role of each arm is interchanged. For gases $n \approx 1^{+}$and we obtain

$$
\Delta t \approx-2 \frac{\left(n^{2}-1\right) L}{c} \frac{v^{2}}{c^{2}}+O\left(v^{4}\right) .
$$


The major significance of this result is that this time difference is not zero when a gas is present in the interferometer, as confirmed by all gas-mode interferometer experiments. Of course this result also shows that vacuum-mode experiments, with $n=1$, will give null results, as also confirmed by experiment. In the case of a gas inclusion of the Fresnel drag effect this results in only a change of sign for $\Delta t$, as shown by comparison of (131) and (140) .

\subsection{Michelson Interferometer and the Einstein Postulates}

The Einstein postulates (58) require that the Michelson interferometer gives a null effect even when operating with a dielectric, whether a gas or a solid. Nevertheless it is interesting to see an explicit demonstration of this ${ }^{35}$.

Let $L=$ proper length of each arm, $V=c / n=$ speed of photon in rest frame of dielectric, and take $c=1$. Relative to 'stationary' observer S: $v=$ speed of interferometer apparatus, $V_{\text {out }}=$ speed of outbound photon in longitudinal arm, $V_{\text {ret }}=$ speed of returning photon in longitudinal arm, $V_{\text {tran }}=$ speed of photon in transverse arm (both ways), $L_{\text {long }}=L\left(1-v^{2}\right)^{1 / 2}=$ length of longitudinal arm, and length of transverse arm $=L$.

Consider the longitudinal arm: The velocity transformation/composition formula gives

$$
\begin{aligned}
V_{\text {out }} & =\frac{V+v}{1+v V} \text { photon \& frame velocities 'add', } \\
V_{\text {ret }} & =\frac{V-v}{1-v V} \text { photon \& frame velocities 'subtract'. }
\end{aligned}
$$

For free-space propagation, $V=1$, these reduce to $V_{\text {out }}=1$ and $V_{\text {ret }}=1$, as required by the second postulate. From these:

$$
\begin{aligned}
& V_{\text {out }}-v=\frac{\left(1-v^{2}\right) V}{1+v V}, \\
& V_{r e t}+v=\frac{\left(1-v^{2}\right) V}{1-v V} .
\end{aligned}
$$

Note parenthetically that

$$
V_{\text {out }}-V_{\text {ret }}=\frac{2 v\left(1-V^{2}\right)}{1-(V v)^{2}},
$$

reducing to 0 for the vacuum $(V=1)$ case.

\footnotetext{
${ }^{35}$ Adapted from notes by Ron Burman.
} 
Hence $\mathrm{S}$ calculates the out and back times in this arm, as in Sect.16.3:

$$
\begin{aligned}
t_{\text {out }} & =\frac{1}{V_{\text {out }}-v} L_{\text {long }} \\
& =\frac{1+v V}{\left(1-v^{2}\right) V} L_{\text {long }} \\
t_{\text {ret }} & =\frac{1}{V(r e t)+v} L_{\text {long }} \\
& =\frac{1-v V}{\left(1-v^{2}\right) V} L_{\text {long }}
\end{aligned}
$$

Adding these and incorporating the Fitzgerald-Lorentz contraction, $\mathrm{S}$ considers the round trip time in this arm to be

$$
t_{\text {trip }}=\frac{2 L}{V}\left(1-v^{2}\right)^{-1 / 2}
$$

Eqn.(148) reducing simply to the vacuum result when $V=1$.

Now consider the transverse arm: A photon in this arm has only a transverse velocity component, $\pm V$, in the rest frame of the apparatus. The velocity transformation formula yields these velocity components in $\mathrm{S}$ (relativistic aberration):

$$
\begin{aligned}
\text { longitudinal component } & =v \\
\text { transverse component } & = \pm V\left(1-v^{2}\right)^{1 / 2} .
\end{aligned}
$$

The relativistic factor arises from time dilation and means that the motion in the transverse arm is relativistically aberrated. Thus $\mathrm{S}$ calculates the photon's resultant speed to be $V_{\text {tran }}$ :

$$
V_{\text {tran }}^{2}-v^{2}=\left(1-v^{2}\right) V^{2}
$$

Using this result, $\mathrm{S}$ calculates the round trip travel time in this arm, as in Sect.16.3

$$
\begin{aligned}
t_{\text {trip }} & =\frac{2 L}{\left(V_{\text {tran }}^{2}-v^{2}\right)^{1 / 2}}, \\
& =\frac{2 L}{V}\left(1-v^{2}\right)^{-1 / 2}
\end{aligned}
$$

Thus the travel times in the two arms, (148) and (152), are identical, whether in a dielectric or not. This is because of a combination of Fresnel drag in the longitudinal arm and relativistic aberration (via time dilation) in the transverse arm. So according to the Einstein postulates the effect is always null and void. While the above derivation may have some validity when the dielectric is a solid, it is clearly in disagreement with experiment when the dielectric is a gas. So the Einstein formalism lacks the contextuality that reality displays. 
Process Studies Supplement 2003 Issue 5

R.T. Cahill.......Process Physics 124

\section{$17 \quad$ References}

\section{References}

[1] D.R. Griffin, Ed., Physics and the Ultimate Significance of Time: Bohm, Prigogine, and Process Philosophy, (State University of NY Press, 1986).

[2] D. Browning and W.T. Myers, Eds., Philosophers of Process, 2nd ed. (Fordham Univ. Press, 1998).

[3] T.E. Eastman and H. Keeton, Resource Guide to Physics and Whitehead, Process Studies Supplement, http://www.ctr4process.org/publications/pss/, 2003.

[4] T.E. Eastman and H. Keeton, Eds., Physics and Whitehead: Process, Quantum and Experience, (SUNY Press, 2003).

[5] D.R. Griffin, Unsnarling the World-Knot: Consciousness, Freedom and the MindBody Problem, (Univ. of California Press, 1998).

[6] R.T. Cahill, Process Physics: Inertia, Gravity and the Quantum, Gen. Rel. and Grav. 34, 1637-1656(2002).

[7] R.T. Cahill, Process Physics: From Quantum Foam to General Relativity, gr-qc/0203015.

[8] R.T. Cahill and C.M. Klinger, Bootstrap Universe from Self-Referential Noise, gr-qc/9708013.

[9] R.T. Cahill and C.M. Klinger, Self-Referential Noise and the Synthesis of ThreeDimensional Space, Gen. Rel. and Grav. 32(3), 529(2000); gr-qc/9812083.

[10] R.T. Cahill and C.M. Klinger, Self-Referential Noise as a Fundamental Aspect of Reality, Proc. 2nd Int. Conf. on Unsolved Problems of Noise and Fluctuations (UPoN'99), Eds. D. Abbott and L. Kish, Adelaide, Australia, 11-15th July 1999, Vol. 511, p. 43 (American Institute of Physics, New York, 2000); gr-qc/9905082.

[11] R.T. Cahill, C.M. Klinger, and K. Kitto, Process Physics: Modelling Reality as Self-Organising Information, The Physicist 37(6), 191(2000); gr-qc/0009023.

[12] R.T. Cahill and K. Kitto, Michelson-Morley Experiments Revisited and the Cosmic Background Radiation Preferred Frame, Apeiron 10, No.2. 104-117(2003); physics/0205070. 
[13] R.T. Cahill, Analysis of Data from a Quantum Gravity Experiment, physics/0207010.

[14] R.T. Cahill, Absolute Motion and Quantum Gravity, physics/0209013.

[15] R.T. Cahill, Absolute Motion and Gravitational Effects, physics/0306196.

[16] R.T. Cahill, Gravity as Quantum Foam In-Flow, physics/0307003.

[17] R.T. Cahill, Quantum Foam In-Flow Theory of Gravity and the Global Positioning System (GPS), physics/0309016.

[18] K. Kitto, Dynamical Hierarchies in Fundamental Physics, p55, in Workshop Proceedings of the 8th International Conference on the Simulation and Synthesis of Living Systems (ALife VIII), E. Bilotta et al., Eds. (Univ. New South Wales, Australia, 2002).

[19] M. Chown, Random Reality, New Scientist, Feb 26, 165, No 2227, 24-28(2000).

[20] B. Müller, J. Reinhardt and M.T. Strickland, Neural Networks - An Introduction, 2nd ed. (Springer, 1991).

[21] Y. Bar-Yam, Dynamics of Complex Systems, (Addison-Wesley, 1997)

[22] E. Nagel and J.R. Newman, Gödel's Proof, (New York Univ. Press, 1995).

[23] G.J. Chaitin, Information, Randomness and Incompleteness, 2nd ed. (World Scientific, 1990).

[24] G.J. Chaitin, The Unknowable, (Springer-Verlag, 1999).

[25] G.J. Chaitin, Exploring Randomness, (Springer-Verlag, 2001).

[26] G. Priest, Beyond the Limits of Thought, (Cambridge Univ. Press, 1995).

[27] F.Th. Stcherbatsky, Buddhist Logic, (Dover Pub. NY, 1962).

[28] J.A. Wheeler Law without Law, in Quantum Theory of Measurement, J.A. Wheeler and W.H. Zurek, Eds. (Princeton Univ. Press, 1983).

[29] P.A. Schilpp, Ed. The Philosophy of Rudolf Carnap, (Cambridge Univ. Press, 1963), p37.

[30] R.T. Cahill, Hadronisation of QCD, Aust. J. Phys. 42, 171(1989); R.T. Cahill, Hadronic Laws from QCD, Nucl. Phys. A 543, 63(1992); R.T. Cahill and S.M. Gunner, The Global Colour Model of QCD for Hadronic Processes: A Review, Fizika $B$ 7, 171(1998). 
[31] G. Parisi and Y. Wu, Perturbation Theory without Gauge Fixing, Scientia Sinica 24, 483(1981).

[32] P. Bak, C. Tang and K. Wiesenfeld, Phys. Rev. Lett. 59, 381(1987); Phys. Rev. A 38, 364(1988).

[33] E. Schrödinger, What is Life?, (Cambridge Univ. Press, 1945).

[34] G. Nicholis and I. Prigogine, Self-Organization in Non-Equilibrium Systems: From Dissipative Structures to Order Through Fluctuations, (J. Wiley \& Sons, NY, 1997).

[35] G. Nagels, A Bucket of Dust, Gen. Rel. and Grav. 17, 545(1985).

[36] J.A. Wheeler, Relativity, Groups and Topology, B.S. Dewitt and C.M. Dewitt, Eds. (Gorden and Breach, New York, 1964).

[37] R.W. Ogden, Non-Linear Elastic Deformations, (Halstead Press, NY, 1984).

[38] N.S. Manton and P.J. Ruback, Skyrmions in Flat Space and Curved Space, Phys. Lett. $B$ 181, 137(1986).

[39] N.S. Manton, Geometry of Skyrmions, Comm. Math. Phys. 111, 469(1987).

[40] T. Gisiger and M.B. Paranjape, Recent Mathematical Developments in the Skyrme Model, Physics Reports 36, 109(1998).

[41] S. Coleman, J.B. Hartle, T. Piran and S. Weinberg, eds., Quantum Cosmology and Baby Universes, (World Scientific, 1991).

[42] I.C. Percival, Quantum State Diffusion, (Cambridge Univ. Press, 1998).

[43] R. Penrose, The Emperors New Mind: Concerning Computers, Minds and the Laws of Physics, (Oxford Univ. Press, 1989).

[44] R.E. Marshak, Conceptual Foundations of Modern Particle Physics, (World Scientific, 1993).

[45] J-J. Dugne, S. Fredriksson, J. Hansson and E.Predazzi, Preon Trinity: A New Model of Leptons and Quarks, 2nd International Conference Physics Beyond The Standard Model: Beyond The Desert 99: Accelerator, Nonaccelerator and Space Approaches, Proceedings, ed. H.V. Klapdor-Kleingrothaus, I.V. Krivosheina. Bristol, IOP, 1236(2000), hep-ph/9909569.

[46] B. Bollabás, Random Graphs, (Academic Press, 1985). 
[47] A. Riess et al., Observational Evidence from Supernovae for an Accelerating Universe and a Cosmological Constant, Astron. J. 116, 1009(1998); S. Perlmutter, et al., Measurements of Omega and Lambda from 42 High Redshift Supernovae, Astrophys. J. 517, 565(1999).

[48] T. Toffoli, in Complexity, Entropy and the Physics of Information, p 301, W.H. Zurek, Ed. (Addison-Wesley, 1990).

[49] A. Unzicker, What Can Physics Learn From Continuum Mechanics?, gr-qc/0011064.

[50] H. Günther, On Lorentz Symmetries in Solids, Physica Status Solidi, A 62, 131(1988).

[51] W.G. Unruh, Experimental Black Hole Evaporation, Phys. Rev. Lett. 46, 1351(1981).

[52] J.S. Bell, Speakable and Unspeakable in Quantum Mechanics, (Cambridge Univ. Press, 1987).

[53] H. Ives, J. Opt. Soc. Am. 29, 183(1939), 38, 413(1939).

[54] R.L. Kirkwood, The Physical Basis of Gravitation, Phys. Rev. 92(6), 1557(1953).

[55] R.L. Kirkwood, Gravitational Field Equations, Phys. Rev. 95(4), 1051(1954).

[56] P. Panlevé, C. R. Acad. Sci., 173, 677(1921).

[57] A. Gullstrand, Ark. Mat. Astron. Fys., 16, 1(1922).

[58] U.R. Fischer and M. Visser, Ann. Phys. 304, 22-39(2003).

[59] N. Ashby Relativity in the Global Positioning System, Living Reviews in Relativity, January 2003; www.livingreviews.org/Articles/Volume6/2003-1Ashby.

[60] P.R. Heyl and P. Chrzanowski, J. Res. Nat. Bur. Standards 29, 1(1942).

[61] L. Facy and C. Pontikis, Comptes Rendus des Scéances de l'Académie des Sciences de Paris 272, Série B, 1397(1971).

[62] J. Renner, in Determination of Gravity Constant and Measurement of Certain Fine Gravity Effects, Y. D. Boulanger and M. U. Sagitov (Eds.), (National Aeronautics and Space Administration, Washington, 1974), pp.26-31

[63] M.U. Sagitov et al., Dok. Acad. Nauk SSSR 245, 567(1979).

[64] G.G. Luther and W. Towler, Phys. Rev. Letters 48, 121(1982). 
[65] J.-Cl. Dousse and Ch. Rhême, Am. J. Phys. 55, 706(1987).

[66] H. de Boer, H. Haars and W. Michaelis, Metrologia 24, 171(1987).

[67] M.A. Zumberge et al., J. Geophys. Res. 95, 15483(1990).

[68] A. Cornaz, B. Hubler and W. Kündig, Phys. Rev. Lett. 72, 1152(1994).

[69] B. Hubler, A. Cornaz and W. Kündig, Phys. Rev. D51, 4005(1995).

[70] M.P. Fitzgerald and T.R. Armstrong, IIIE Trans. Instrum. Meas. 44, 494(1995).

[71] C.H. Bagley and G.G. Luther, Phys. Rev. Lett. 78, 3047(1997).

[72] J. Luo et al., Phys. Rev. D59, 042001(1998).

[73] J. P. Schwarz et al., 1998, Science 282, 2230(1998).

[74] O.V. Karagioz, V.P. Izmaylov and G.T. Gillies, Grav. Cosmol. 4, 239(1998).

[75] J. Schurr, F. Nolting and W. Kündig, Phys. Rev. Letters 80, 1142(1998).

[76] M.P. Fitzgerald and T.R. Armstrong, Meas. Sci. Technol. 10, 439(1999).

[77] F. Nolting, J. Schurr, S. Schlamminger and W. Kündig, Meas. Sci. Technol. 10, 487(1999).

[78] S.J. Richman, T.J. Quinn, C.C. Speake and R.S. Davis, Meas. Sci. Technol. 10, 460(1999).

[79] U. Kleinevoss, H. Meyer, A. Schumacher and S. Hartmann, Meas. Sci. Technol. 10, 492(1999).

[80] J. H.Gundlach and S.M. Merkowitz, Phys. Rev. Letters 85, 2869(2000) and SISSA preprint gr-qc/0006043.

[81] T.J. Quinn, C. C. Speake, S. J. Richman, R. S. Davis and A. Picard, Phys. Rev. Lett. 87, 111101(2001).

[82] P. Baldi et al., Phys. Rev. D64, 082001(2001).

[83] J.P. Mbelek and M. Lachièze-Rey, Grav. Cosmol. 8331-338(2002).

[84] G.T. Gillies, The Newtonian Gravitational Constant: Recent Measurements and Related Studies, Rep. Prog. Phys. 60, 151-225(1997). 
[85] Descartes, Principles of Philosophy, V.R. Miller and R.P. Miller (trans), (Reidel Publishing, 1983).

[86] I. Newton, Principles of Natural Philosophy, 1729, trans. by A. Motte, (Dawsons).

[87] A.A. Michelson, Amer. J. Sci. S. 3 22, 120-129(1881).

[88] A.A. Michelson and E.W. Morley, Philos. Mag. S.5, 24, No. 151, 449-463(1887), (available at http://www.scieng.flinders.edu.au/cpes/people/cahill_r/processphysics.html)

[89] D.C. Miller, The Ether-Drift Experiment and the Determination of the Absolute Motion of the Earth, Rev. Mod. Phys. 5, 203-242(1933), (available at http://www.scieng.flinders.edu.au/cpes/people/cahill_r/processphysics.html)

[90] R.S. Shankland, S.W. McCuskey, F.C. Leone and G. Kuerti, New Analysis of the Interferometer Observations by Dayton C. Miller, Rev. Mod. Phys. 27(2), 167178(1955).

[91] K.K. Illingworth, Phys. Rev. 30, 692-696(1927).

[92] G. Joos, Ann. d. Physik [5], 7, 385(1930).

[93] W.M. Hicks, Phil. Mag, [6], 3, 256, 555(1902); 9, 555(1902).

[94] G.F. Fitzgerald, Science, 13, 420(1889).

[95] H.A. Lorentz, Electric Phenomena in a System Moving With Any Velocity Less Than That of Light, in The Principle of Relativity, (Dover, 1952).

[96] J. Larmor, Phil. Trans. Roy. Soc. London, 190-205(1897).

[97] L. Swenson, The Ethereal Aether: A History of the Michelson-Morley-Miller Aether Drift Experiments, (U. Texas Press, 1972).

[98] J. DeMeo, Dayton Miller's Ether-Drift Experiments: A Fresh Look, http://www.orgonelab.org/miller.htm

[99] R.W. Clark, Einstein: The Life and Times, (World Publishing C., 1971).

[100] H.A. Munéra, Aperion 5, No.1-2, 37-54(1998).

[101] C. Lineweaver et al., Astrophysics J. 470, 38(1996).

[102] T.S. Jaseja, A. Javan, J. Murray and C.H. Townes, Test of Special Relativity or Isotropy of Space by Use of Infrared Masers, Phys. Rev. A 133, 1221(1964). 
[103] R. DeWitte, http://www.ping.be/ pin30390/.

[104] D.G. Torr and P. Kolen, in Precision Measurements and Fundamental Constants, B.N. Taylor and W.D. Phillips, Eds. Natl. Bur. Stand.(U.S.), Spec. Publ. 617, $675(1984)$.

[105] H.P Kennedy and E.M. Thorndike, Phys. Rev. 42, 400(1932).

[106] A. Brillet and J.L. Hall, Phys. Rev. Lett. 42, No.9, 549-552(1979).

[107] C. Braxmaier, H. Müller, O. Pradl, J. Mlynek, A. Peters and S. Schiller, Phys. Rev. Lett. 88, 010401(2002).

[108] J.A. Lipa, J.A. Nissen, S. Wang, D.A. Striker and D. Avaloff, Phys. Rev. Lett. 90, 060403-1(2003).

[109] H.P. Robertson, Rev. Mod. Phys., 21, 378(1949).

[110] R.M. Mansouri and R.U. Sexl, J. Gen. Rel. Grav., 8, 497(1977), 8, 515(1977), 8, 809(1977).

[111] J. Shamir and R. Fox, Il Nuovo Cimenta, LXII B N.2, 258(1969).

[112] G.G. Stokes, Phil. Mag. 27, 9(1845).

[113] W. Voigt. An English translation and commentary on Voigt's paper are found in A. Ernst and Jong-Ping Hsu, First Proposal of the Universal Speed of Light by Voigt in 1887, Chinese Journal of Physics, 39, No. 3, 211-230(2001).

[114] C.J. Bjerknes, Albert Einstein. The Incorrigible Plagiarist, (XTX, Inc. Il. USA, 2002).

[115] R. Shankland, Applied Optics, 12(10), 2280-2287(1973).

[116] M. Allais, AeroSpace Eng., Sept.-Oct. 18, p.46(1959); http://allais.maurice.free.fr/English/Science.htm

[117] E.J. Saxl and M. Allen, 1970 Solar Eclipse as "Seen" by a Torsional Pendulum, Phys. Rev., D3, 823(1971).

[118] S.W. Zhou and B.J. Huang, Abnormalities of the Time Comparisons of Atomic Clocks during the Solar Eclipses, Il Nuovo Cimento, 15C, N.2, 133(1992).

[119] S.W. Zhou, B.J. Huang and Z.M. Ren, The Abnormal Influence of the Partial Solar Eclipse on December 24th, 1992, on the Time Comparisons between Atomic Clocks, Il Nuovo Cimento, 18C, N.2, 223(1995). 
[120] S.W. Zhou, Abnormal Physical Phenomena Observed when the Sun, Moon and Earth are Aligned, 21st Century Science and Technology, pp.54-61, Fall 1999.

[121] J.D. Anderson, et al., Phys. Rev. Lett. 81, 2858(1998).

[122] F.D. Stacey et al., Phys. Rev. D23, 2683(1981).

[123] S.C. Holding, F.D. Stacey, and G.J. Tuck, Phys. Rev. D33, 3487(1986).

[124] S.C. Holding and G.J. Tuck, Nature (London) 307, 714(1987)

[125] F.D. Stacey, G.J.Tuck, G.I. More, S.C. Holding, B.D. Goodwin and R. Zhou, Geophysics and the Law of Gravity, Rev. Mod. Phys. 59, 157(1987).

[126] J. Thomas, et al., Testing the Inverse-Square Law of Gravity on a 465-m Tower, Phys. Rev. Lett., 63, 1902(1989).

[127] C. Jekeli, D.H. Eckhardt, and A.J. Romaides, Phys. Rev. Lett. 64, 1204 (1990).

[128] R.T. Cahill, Smart Nanostructures and Synthetic Quantum Systems, Proceedings of SPIE Conference No. 4590, L.B. Kish, Ed. 319-328(2001); R.T. Cahill, Synthetic Quantum Systems, Smart Materials and Structure, 11, 699-707(2002); physics/0209064.

[129] P. Ripota, Das Universum hat ein Bewusstsein, P.M. Magazin, pp.20-25,September 2003. 